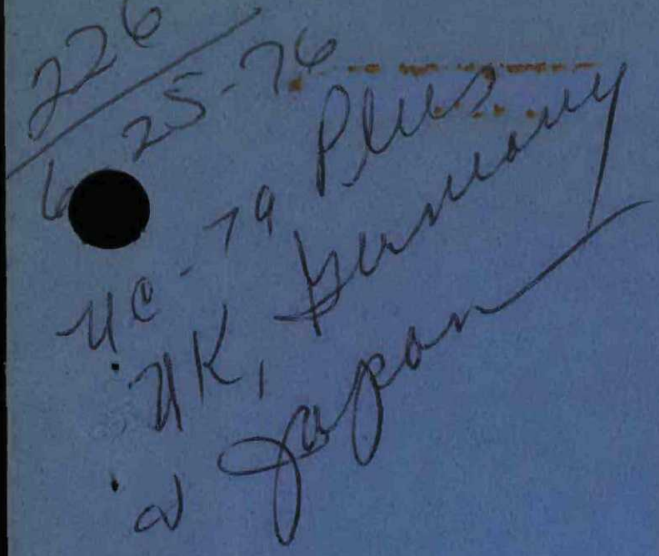

HEDL-TME 75-43

UC $-79, b$

REACTOR MATERIALS PERFORMANCE

PREDICTIONS USING DAMAGE

FUNCTIONS: ANALYSIS OF FLUENCE

LIMIT UNCERTAINTIES

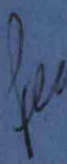

P.O. Box 1970 Richland, WA 99352

HANFORD ENGINEERING DEVELOPMENT LABORATORY

Operated by Westinghouse Hanford Company

A Subsidiary of Westinghouse Electric Corporation

Prepared for the U.S. Energy Research and Development MASTER Administration under Contract No. E[45-1)-2170 


\section{DISCLAIMER}

Portions of this document may be illegible in electronic image products. Images are produced from the best available original document. 


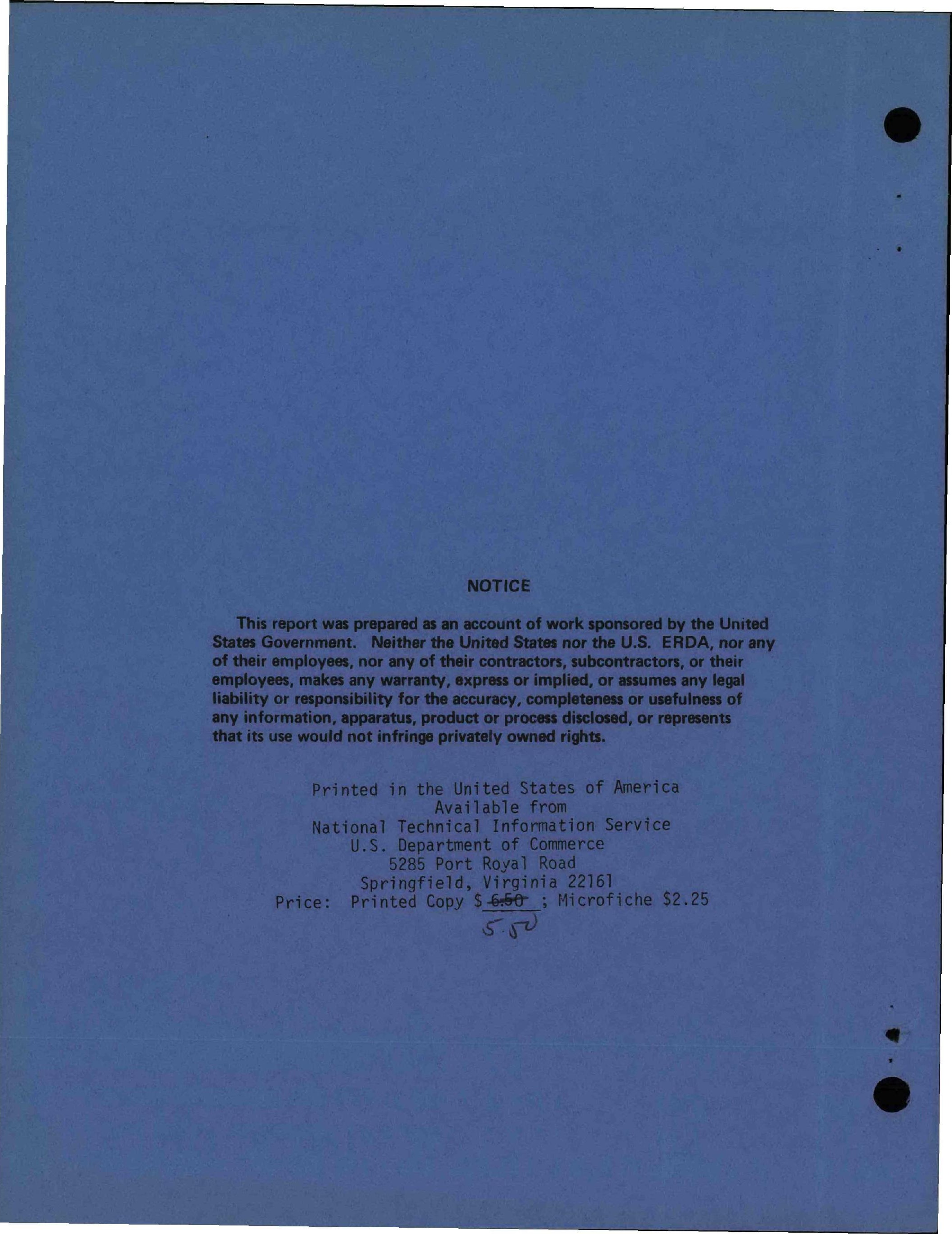




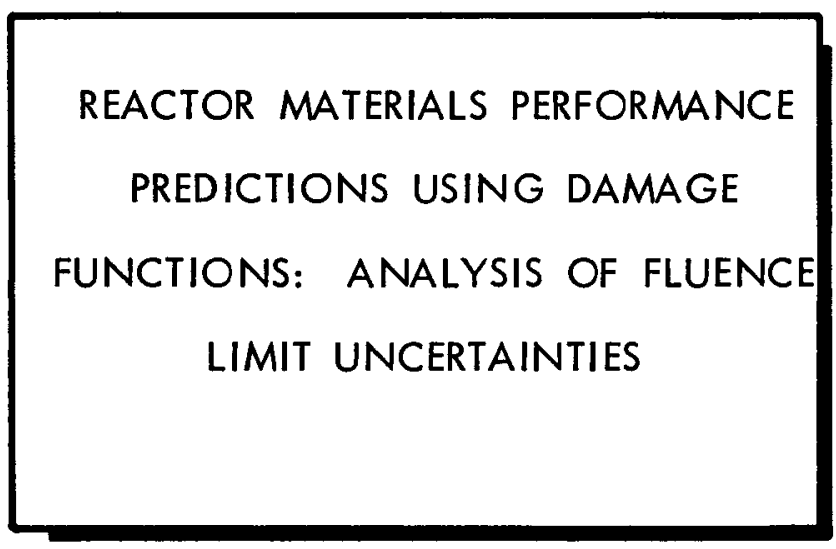

G. R. Odette

R. L. Simons

W. N. McElroy

This report was prepoTICE

This report was prepared as an account of work

the United States nor the United States. Nenther

Research and Development United States Energy

their employees, nor any of then, nor any of

unbcontractors, or their employees, contractors,

warmenty, express or impled, or assumes any any

liability or responsibility for the accuracy, completerest

or usefulnes of any information, apparatus, product or

January, 1976

\section{Hanford Engineering Development Laboratory}

\begin{tabular}{|c|c|c|}
\hline $\begin{array}{l}\text { Operated by the } \\
\text { Westinghouse } \\
\text { Hanford Company }\end{array}$ & $\begin{array}{l}\text { A Subsidiary of } \\
\text { Westinghouse Electric } \\
\text { Corporation }\end{array}$ & $\begin{array}{l}\text { for the United States } \\
\text { Energy Reseairch and } \\
\text { Development Administration } \\
\text { Contract No. E(45-1)2170 }\end{array}$ \\
\hline
\end{tabular}




\title{
REACTOR MATERIALS PERFORMANCE PREDICTIONS USING DAMAGE FUNCTIONS: ANALYSIS \\ OF FLUENCE LIMIT UNCERTAINTIES
}

\author{
G. R. Odette \\ R. L. Simons \\ W. N. McElroy
}

\begin{abstract}
Some limits to appropriate application and the characteristics of uncertainties in damage function analysis were investigated by means of computer experiments. Simulated irradiations in available neutron spectra were evaluated in terms of simple damage models and were used to study 1) the existence of domage functions, 2) the uniqueness of damage function solutions, 3) data error propagation, and 4) procedures for combining various errors to provide a total lower bound fluence limit for a specified property change. Not surprisingly, an important factor in achieving a successful domage function analysis was found to be the similarity between the experimental spectra which were used to generate the damage function and the spectra in which it was applied.
\end{abstract}


$\bullet$

$\Delta$ 


\section{CONTENTS}

$\underline{\text { Page }}$

LIST OF FIGURES vi

LIST OF TABLES viii

I. INTRODUCTION 1

A. Review 1

B. Scope of Present Study 3

II. SUMMARY AND CONCLUSIONS 5

III. RIGOROUS EXISTENCE OF G(E) 7

IV. ANALYTICAL METHODS AND DATA TREATMENT 21

A. SAND-II Algorithm 21

B. Data Extrapolation 24

V. TRIAL SOLUTIONS AND SOLUTION ERROR 27

A. Uniqueness Uncertainty 27

B. Damage Models and Energy Dependence 35

VI. DATA ERROR PROPAGATION 42

VII. TOTAL SOLUTION ERROR AND FLUENCE LIMIT ASSIGNMENT 51

VIII. RECOMMENDATION FOR FUTURE WORK 59

IX. ACKNOWLEDGEMENTS 61

X. REFERENCES 63

APPENDIX A. A MODEL FOR HELIUM-DISPLACEMENT INTERACTION A-1

APPENDIX B. MODIFICATIONS TO THE SAND-II CODE B-1

APPENDIX C. DFA OVERVIEW AND CHARACTERISTICS OF SPECTRA EMPLOYED IN THIS STUDY C-

APPENDIX D. SOME TRIAL SOLUTIONS FOR DISPLACEMENT AND HEL IUM GENERATION PROCESSES

APPENDIX E. APPLICATION OF ERROR ANALYSIS TO DAMAGE FUNCTIONS FOR YIELD STRENGTH IN STAINLESS STEEL AND IRON 


\section{FIGURES}

Figure

Page

1 Calculated Interactive Model Damage Efficiency Functions

2 Calculated and Unfolded Effective Damage Efficiency Function for Production of $10^{16}$ Defects $/ \mathrm{cm}^{3}$ and Calculated Response Ranges for Three Test Spectra

$3 \quad$ Calculated and Unfolded Effective Damage Efficiency Function for Production of $10^{16}$ Defects $/ \mathrm{cm}^{3}$ and Calculated Response Ranges for Three Design Spectra

4 Input and Solution Effective Damage Efficiency Functions for Production of $10^{16}$ Defects $/ \mathrm{cm}^{3}$

5 Solution Damage Functions to Produce $10^{16}$ Defects $/ \mathrm{cm}^{3}$ Using Reduced Spectral Coverage

6-a Damage Efficiency Function Uniqueness Study, $b=+0.4$ and $\mathrm{C}=10$

6-b Damage Efficiency Function Uniqueness Study, $b=-0.4$ and $\mathrm{C}=10$

7-a Solution Uniqueness Study by Comparing the Fluence Limit Ratios of True/Input and True/Solution for the FTR Design Spectra

7-b Solution Uniqueness Study by Comparing the Displacement Rate Ratios of Input/True and Solution/True for the FTR Design Spectra

8-a One Standard Deviation Error Limits in the Displacement Cross Section Due to Various Combinations of Integral and Differential Errors

8-b One Standard Deviation Error Limits in the Displacement Cross Section Due to Various Combinations of Integral and Differential Errors

8-c One Standard Deviation Error Limits in the Displacement Cross Section Due to Various Combinations of Integral and Differential Errors

9 A Study of Systematic Errors by Perturbation of the Thermal Reactor Data $\pm 20 \%$

10-a Monte Carlo Analysis of Combined Data and Uniqueness Errors Using a Parameter Variation Approach (Procedure A)

10-b Monte Carlo Analysis of Combined Data and Uniqueness Errors Using a Least Square Fit Approach (Procedure B) 


\section{FIGURES (Cont'd)}

Figure

Page

11 Nominal and Lower Bound Fluence Limits to Attain Equal

Displacement Damage in the FTR Design Spectra

B-1 Logic Used in the SAND-II Monte Carlo Analysis B-5

C-1 Goal: Predictions From Test Irradiation Data of Material Performance During Application

C-2 Schematic Flow Diagram of the Investigation

C-3 APFACC--Normalized Flux per Unit Lethargy Versus Energy EP5DUM--Normalized Flux der Unit Letharay, Versus Energy ETRG7--Normalized Flux per Unit Lethargy Versus Energy

C-7 EBR-D--Normalized Flux per Unit Lethargy Versus Energy

D-1 Displacement Cross Section for $18 / 8(\mathrm{Cr} / \mathrm{Ni}$ Stainless Steel Using $40 \mathrm{eV}$ and $10 \mathrm{keV}$ thresholds

D-2 Residual Defect Cross Sections for Stainless Steel

D-5 Stainless Steel Displacement Production Response Functions Versus Lethargy for the Three FTR Design Spectra

D-6 Stainless Steel Helium Production Response Functions Versus Lethargy for the Three FTR Design Spectra

D-7 Stainless Steel Helium and Displacement Response Functions Versus Energy for the ETR Spectrum 
$\underline{\text { Table }}$

Page

1 A Comparison of Damage Level/Unit Fluence (True/ Predicted From EDFs) in FTR Spectra Calculated

From the Interactive Damage Model and the Effective Damage Function

2 Solution Uniqueness Study by Comparing the Fluence Limit Ratios of Input/True $\left(R_{I}\right)$ and Solution/True $\left(R_{F}\right)$ for FTR Design Spectra

3 Monte Carlo Percent Negative Standard Deviation in FTR Design Spectra for Displacement Rates Using Various Combinations of Group Averaged Spectral Errors, $\delta \phi_{i}$, and Integral Data Errors, $\delta M_{i}$

$4 \quad$ Combined Monte Carlo and Uniqueness Percent Negative Standard Deviation (\%) Errors in the Displacement Rate in the Three FTR Test Spectra

A-1 Data for and Predictions of Interactive Model

C-1 Integral Parameters Characterizing the Spectra Used in the Computer Studies

$C-6$

E-1 Percent Errors Assigned to EBR-II Spectra E-3

E-2 Group Averaged Damage Function in the ETR Spectrum E-4

E-3 Estimated Uncertainties (95\% Confidence Leve1) in Predicted Fluence Required to Attain $60 \mathrm{ksi}$ Yield Strength

$E-5$

E-4 Empirical Damage Function for Iron E-6

E-5 Exposures Needed to Produce $15 \mathrm{ksi}$ Yield Strength Changes $\times 10^{-19}\left(\mathrm{n} / \mathrm{cm}^{2}\right)$ 
REACTOR MATERIALS PERFORMANCE PREDICTION:

\section{A CRITIQUE OF DAMAGE FUNCTION ANALYSIS}

\section{INTRODUCTION}

\section{A. Review}

Variables which influence the irradiation behavior of materials include material type, purity and substructure, irradiation conditions, and test conditions. In some advanced reactor types (e.g., LMFBR's and CTR's) novel neutron flux spectra will be encountered emphasizing the need to account properly for spectral effects. Emphasis is placed on the spectral variable because it is difficult to duplicate design spectra in test environments. Furthermore, the significant ranges of other variables are more easily encompassed in test environments. Specifically, spectral characteristics must be considered if damage exposure units are to be properly defined. Use of spectral dependent damage units that are based on fluxes above an energy threshold can be highly inappropriate. (1) Multiple parameter correlation procedures using spectral indices are undesirable if they lack a direct physical basis. (2) Estimates of spectral influence on damage may also be based on simple damage models, most notably total displacement calculations. (3) However, the absence of a detailed theoretical understanding of damage mechanisms may introduce large uncertainties in damage predictions if they are based solely on such calculations.

To mitigate the problems outlined above, the semi-empirical technique of damage function analysis (DFA) has been employed to estimate the damaging efficiency of neutrons of various energies*. DFA allows the use of both the best available theoretical and experimental information in estimating the neutron energy (or spectral) dependence of radiation damage. This is accomplished through experimental measurements carried out on materials irradiated in a variety of neutron spectra in which all other significant variables (e.g., temperature, composition, etc) are ideally kept constant. However, precise control and determination of important

\footnotetext{
*A schematic description of the procedure for deriving and applying damage
} functions is included in Appendix $\mathrm{C}$. 
variables such as temperature may in fact be difficult. Uncertainties due to these factors must be treated in the analysis and, if large, may prevent application of DFA (and of any alternate method of data correlation). Damage functions have been determined for a number of materials, properties and temperatures. $(4-14)$

The derivation of a damage function, treated in greater detail below, is fairly straightforward. An irradiation induced property change is measured in several neutron environments whose flux magnitudes $\Phi$, normalized spectra $\phi(E)\left[\int_{0}^{\infty} \phi(E) d E=1\right]$, and irradiation times $t$ have been characterized. The total fluence in each of the spectra needed to effect a common property level, $P$, is determined by extrapolating or interpolating the measured data to the property level $P$ using the appropriate fluence dependence for each spectrum. The measured quantity $M_{j}$ in the $i^{\text {th }}$ spectrum is taken as $M_{j}=P / \Phi t_{i} . M_{j}$ is assumed to be related to the spectral shapes in the from of a set of integral equations as

$$
M_{i}=\int_{0}^{\infty} \phi_{i}(E) G(E) d E, i=1, \ldots, n .
$$

where $\mathrm{n}$ is the number of spectra.

Unfolding of the damage function $G(E)$ using the SAND-II code (see Section IV) is achieved by an iterative perturbation of an input trial solution, $G^{\circ}(E)$, to obtain integral agreement with the experimental measurements. The trial solution $G^{\circ}(E)$ is one means of inserting physically based theoretical information into the semi-empirical DFA. The displacement cross section is most often used for $G^{\circ}(E)$. In the limit of a single measurement of $P$ in a single spectrum, the derived $G(E)$ has the same form as $G^{\circ}(E)$ but with a magnitude which is set by the experimental value of $P /(\Phi t)$.

Damage functions are used to estimate the fluence $\Phi t_{j}$ needed to produce $P$ in any (normalized) spectrum $\phi_{j}(E)$. That is, $G(E)$ allows the definition of equivalent spectral dependent exposures. In particular, $P$ may be the maximum permissible change in some property and $\Phi t_{j}$ the corresponding limiting fluence. This may be obtained from 


$$
\Phi t_{j}=P / \int_{0}^{\infty} \phi_{j}(E) G(E) d E .
$$

Note that the $G(E)$ was derived for a specific set of nonspectral parameters, i.e., a single material, irradiation and test temperature, strain rate, etc., and thus its application is limited to the same set of nonspectral parameters. If the fluence limit for a different property level or other nonspectral parameter is desired, a new damage function must be derived.

B. Scope of Present Study

Two critical and related questions concerning DFA are: What are the situations in which the technique can be reasonably applied, and what are the magnitudes and consequences of the errors in derived $G(E)$ 's and the fluence limits determined from them? Only partial investigations of these questions have been reported in previous DFA work.

The present study included consideration of the following:

1. Rigorous existence of $G(E)$ in the form implied in equation (1).

2. Uniqueness and appropriateness of DFA solutions. With available data a direct mathematically unique solution of the integral equation is not as useful as an iterative indirect procedure which starts with an approximate effective damage function (EDF). (15) Thus, the solution is to some extent affected by the input form, leading to a problem of mathematical uniqueness.

3. Data error propagation. Errors in the data may be significantly multiplied in the analysis.

4. Error combination to provide total fluence limit uncertainties. Assessment of damage function and fluence limit uncertainties, which are inherent in any data correlation technique is critical to their effective utilization. DFA offers a powerful means of systematically estimating the magnitude of EDF errors and their consequences in terms of fluence limit 
uncertainties and fluence limit lower bounds. No other technique developed to date appears to allow combination of data, model, and analytical uncertainties to be propagated to fluence limit error estimates.

To study the appropriateness and accuracy of DFA, a series of computer studies was carried out using damage levels calculated from some simple damage models in place of actual irradiation effects data. Synthetic data were generated for seven existing spectra including EBR-2 midplane $(z=0 \mathrm{~cm})$ Rows 2 and 7, EBR-2 $(z=59 \mathrm{~cm})$ Rows 2 and $7 *$, Godiva, ETR and CP5 Dummy. These are referred to as "test spectra" for which data are available or could be generated. The $G(E) s$ resulting from DFA were used to predict damage rates or levels in three calculated Fast Test Reactor (FTR) spectra for comparison with the true values calculated from the damage models. These calculated spectra, corresponding to core, grid plate, and vessel locations, are referred to as "design spectra" and are characteristic of environments in which damage functions would be applied for engineering prediction. The FTR grid plate and vessel spectra are appreciably softer than available fast reactor spectra in which data can be generated, having mean energies of 0.04 and $0.002 \mathrm{MeV}$ respectively. Such integral comparisons give a direct measure of the ability of DFA to determine fluence limits, which is its main engineering function. A flow diagram of these computer studies, the artificial data used, and plots of the spectra are given in Appendix C.

The conclusions drawn from this study, which was necessarily 1 imited in both the spectra and damage models considered, cannot be completely generalized. However, the results should be generally indicative of the situations encountered in a fission reactor and, particularly, LMFBR development. They provide guides to proper DFA application and provide some estimates of the uncertainties inherent in such analysis. Furthermore, procedures are outlined which can be used to estimate actual damage function errors and uncertainties in their application to the prediction of fluence limits.

*These EBR-2 spectra will be referred to subsequently as EBR-A, B, C, and $D$, respectively. 


\section{SUMMARY AND CONCLUSIONS}

Extensive DFA computer studies have been carried out for simulated irradiations in available neutron spectra based on simple damage models. The accuracies of the EDF's were generally evaluated as integral responses in a wide range of typical fast spectra. The study indicated that DFA, properly used, is a useful method of combining both theoretical and experimental information to determine the effective neutron energy dependence of radiation damage. Comparison with other methods (such as $F>0.1 \mathrm{MeV}, \bar{E}$, etc) showed that EDF's provide substantial improvements in estimating directly the nominal fluence limits in environments for which data are not available.

Perhaps more importantly, DFA can be used to estimate lower bound fluences by systematic propagation of data errors and uncertainties in solution uniqueness. Error propagation studies showed that the EDF solution is relatively insensitive to spectral shape errors, moderately sensitive to data errors and very sensitive to the number and response energy range of the test spectra. Fluence prediction errors will be relatively small and will be dominated by data errors if the spectra to which EDF's are applied are similar, or have similar response ranges, to those in which they were derived. Not surprisingly, in situations where large spectral extrapolations are demanded, the errors may be large and are dominated by solution nonuniqueness and questions of solution existence. Error magnification is possible in energy ranges of EDF's where there is low damage response. These results emphasize the need for material property change measurements in the widest range of available spectra, in order to minimize errors in fluence limit estimates.

DFA is most appropriate if the damage process is dominated in all spectra by one mechanism, such as displaced atoms. For example, an analysis might start with $G^{\circ}(E)=G_{D}(E)$ (a normalized displacement cross section), but the resulting EDF may vary from $G_{D}(E)$ in such a way as to indicate that clustered or freely migrating defects rather than total displacements are the primary source of damage. Interactions between more than one defect type, or rate effects, may invalidate the assumptions in DFA as contained in Equation ( 1$)$. That is, $a G(E)$ independent of flux-spectrum 
may not exist as such. The effect of such phenomena on the accuracy or appropriateness of DFA is difficult to quantify. A specific example investigated in this study involved first order interactions between helium and displaced atom damage. It indicated that DFA was still able to supply a reasonable and conservative estimate of the nominal fluence limits for FTR grid plate and vessel design spectra which are very different from the test environments from which the EDF was derived. This was true only if the appropriate $G^{\circ}(E)$ and thermal reactor data were used in the analysis. The prediction was very good for the FTR core spectrum, which is similar to the test spectra--a result which should have general validity.

It is not possible, however, to generalize extensively from this single interactive damage model. Uncertainties in DFA due to questions of existence can be evaluated only with the help of damage models or more data when available. Extensive spectral extrapolation (for example, direct use of fission EDFs in fusion spectra) naturally involves large uncertainties because the dominant damage mechanism may change, but may also be superior to other correlation techniques (this is suggested in this limited study). It is clear, however, that developing better damage models and generalized means of analyzing radiation effects data generated in meaningful experiments should continue to be a primary goal of radiation damage research. 


\section{RIGOROUS EXISTENCE OF $G(E)$}

It should be stated at the outset that the rigorous existence of $G(E)$ is not necessarily a prerequisite for useful application of DFA. In recognition of this fact the term effective damage function (EDF) will often be used in the following discussion.

The basic problem of damage function existence can be stated as follows: If neutrons of one energy can influence the damage efficiency of neutrons of other energies, then the approach is invalid in a rigorous sense $[G(E)$ and $\phi(E)$ cannot be separated as in Equation (1)]. The damage efficiency of a neutron is recognized to be dependent, in principle, not only on its energy, but also on the duration and temperature of irradiation, flux level, energies of other neutrons (spectrum), initial material condition, and existing damage state, including that which is a result of effects such as thermal aging.

Irradiation damage is the consequence of the neutron induced production of atomic level defects in the form of primary displacements, stable point or clustered defects, mobile defects, transmutants and microstructural alterations (such as phase precipitation or solutioning). Rigorous adherence of the damage function approach to Equation (1) implies that a property level change is uniquely related to the defect state which exists in the material. Furthermore, it implies that this state evolved as the result of the generation of a single dominant neutron induced type of defect (e.g., displaced atoms) with a definable energy response.

Another problem with damage function analys is is the inherent variation in flux levels even if it is possible to keep all other parameters except spectrum constant. That is, it is assumed that total fluence is the appropriate spectral dependent exposure parameter as opposed to independent treatment of flux and time. Rate effects may be significant and their influence should be carefully examined.

An example of a significant situation of interest where a damage function does not rigorously exist, but an EDF may be nevertheless usefuT, is when displacement damage and transmutant helium gas interact synergistically. 
To study first order helium-displacement interaction, a simple model was adopted which will subsequently be referred to as the "interactive model". It assumes that damage results only from the first order interaction between helium and vacancies (displaced atoms). The damage level is proportional to $(\Phi t)^{2} \cdot \bar{\sigma}_{H i} \cdot \bar{\sigma}_{D i}$ where $\bar{\sigma}_{H i}$ and $\bar{\sigma}_{D i}$ are spectral averaged cross sections for helium generation and displacements respectively. A material consisting of $1 \mathrm{ppm} B^{10}$ in pure iron was used in this study. An energy dependent form of this "interactive model" is

$$
G_{j}^{\prime}(E, \phi)=K \cdot \Phi t \cdot\left[\bar{\sigma}_{H_{j}} \cdot \sigma_{D}(E)+\bar{\sigma}_{D_{j}} \cdot \sigma_{H}(E)\right]
$$

where $\sigma_{H}(E)$ and $\sigma_{D}(E)$ are the energy dependent helium and displacement cross sections respectively, and $K$ is a proportionality constant (see Appendix A). It can be seen that no single energy dependent function exists for all spectra, but each depends explicitly on the $i^{\text {th }}$ spectral shape. Several of these functions are shown in Figure 1 for some of the spectra.

A damage function does not rigorously exist in this circumstance since one type of defect produced by neutrons of one energy can interact with different defects generated at other energies to produce damage.* To investigate the effectiveness of DFA in such a situation, a series of standard unfoldings was carried out. The data used in Equation (1) were damage level per unit fluence calculated from the "interactive model" for each of the $i$ test spectra (see Appendix A). Two types of noninteractive input (i.e., trial solution) were used in the analysis, viz., a "total displacement" cross section for stainless steel from Doran ${ }^{(3)}\left[G_{D}(E)\right]$ and a least squares fit to the data of the function

$$
G^{\circ}(E)=A \cdot W_{1}(E) \cdot E^{b}+C \cdot W_{2}(E)
$$

where $A, b$, and $C$ are fitted constants. In this case $W_{1}(E)=1.0, W_{2}(E)=1 / \sqrt{E}$. The weighting function $W_{2}(E)$ is used to account for He production by low energy neutrons.

*See Section on Damage Models and Energy Dependence. 


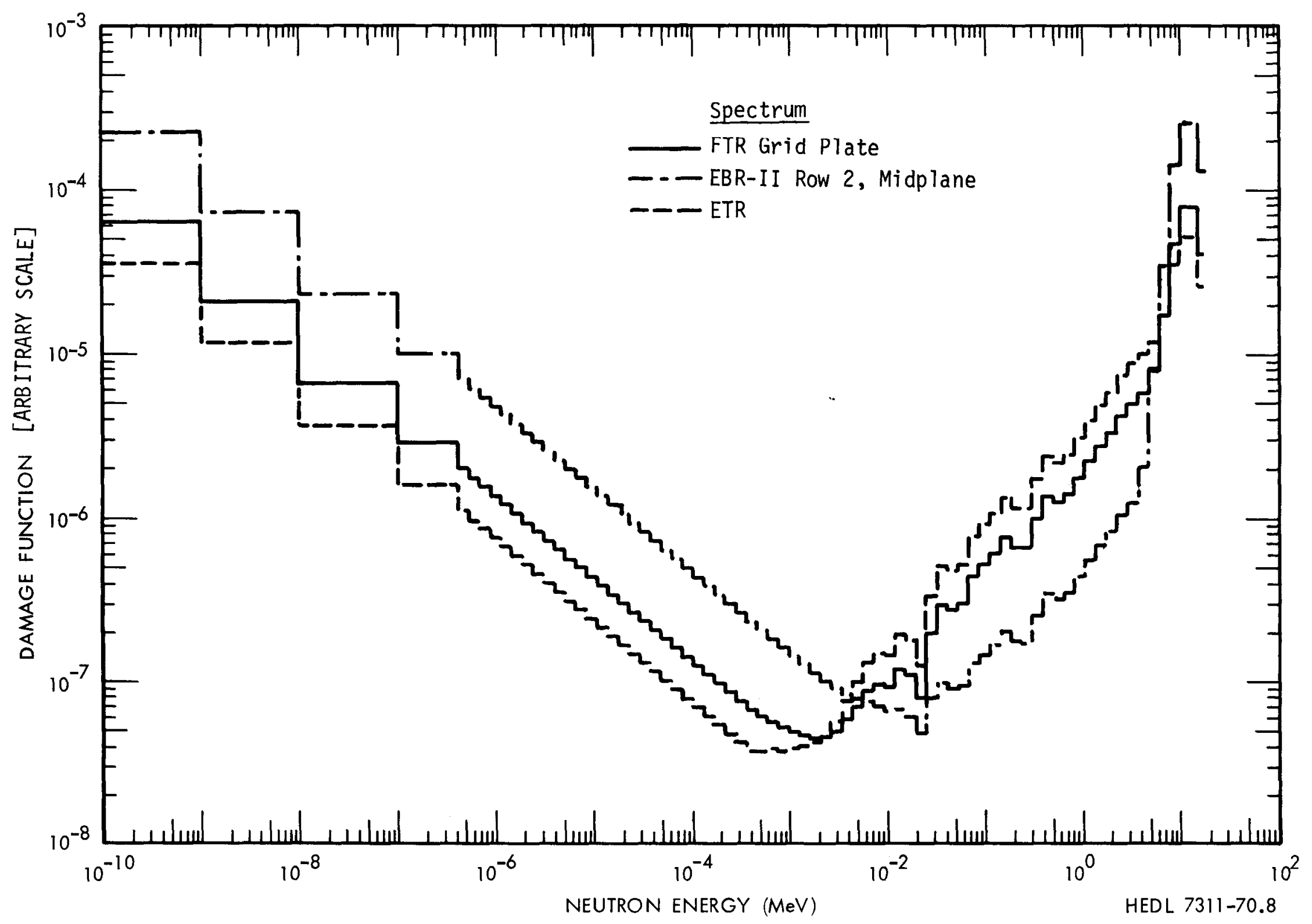

FIGURE 1. Calculated Interactive Mode1 Damage Efficiency Functions. 
An integral comparison of the effectiveness of the DFA method in predicting the fluence required to attain a given damage level in the design spectra are shown in Table 1. The comparison is made by taking the ratio of true damage/unit fluence (based upon interactive model) to estimated damage/unit fluence (based on EDF shape). Note that since the damage level is the same for both true and estimated results the ratio is that of estimated fluence to true fluence. A ratio of 1 indicates exact agreement between the EDF and the model, a ratio > 1 indicates a high predicted fluence limit (non-conservative) and a ratio $<1$ indicates a low predicted fluence limit (conservative). The comparison was made with several EDF's derived in this computer study. These included the solution starting from the displacement cross section input, the solution starting from the least squares fit input, and the least squares input itself. Table 1 also gives a comparison of other correlation procedures applied to the interactive model data. These include mean neutron energy $(E)$, total fluence ( $E>0 \mathrm{MeV})$, and fast fluence $(E>0.1 \mathrm{MeV})$. A regression analysis, based on Lynch's ${ }^{(2)}$ approach, was also studied with the aid of the OMNITAB regression analysis program. (16) This method uses a polynomial form where the independent variables are activation fluences (i.e., total fluence times the spectral averaged cross section) of various foil monitors, and temperature.

Since the seven test spectra represent the ideal situation in terms of spectral coverage, several cases were redone with the four fast reactor spectra only. These cases are also shown in Table 1.

The results of Table 1 can be understood by examination of Figure 2-4. In Figure 2, the derived effective damage functions and the calculated damage efficiency functions for representative hard, intermediate and thermal test spectra are shown. The calculated functions are plotted only in energy 
TABLE 1

A COMPARISON OF DAMAGE LEVEL/UNIT FLUENCE (TRUE/PREDICTED FROM EDF's)

IN FTR DESIGN SPECTRA CALCULATED FROM THE INTERACTIVE

DAMAGE MODEL AND THE EDF's*

Ratio of True to Calculated Damage Level/Unit Fluence

\begin{tabular}{|c|c|c|c|c|c|c|}
\hline \multicolumn{3}{|c|}{ For Different Starting $G^{\circ}(E)$} & \multicolumn{4}{|c|}{ For 0ther Correlation Procedures } \\
\hline $\begin{array}{c}\text { Displacement } \\
\text { Cross } \\
\text { Section } \\
\text { After } 50 \\
\text { Iterations }\end{array}$ & $\begin{array}{c}\text { Least } \\
\text { Squares } \\
\text { Fit** } \\
\text { After } 50 \\
\text { Iterations }\end{array}$ & $\begin{array}{r}\text { Least Squares } \\
\text { Fit Input** }\end{array}$ & $\begin{array}{c}\text { Mean } \\
\text { Energy } \\
\bar{E} \quad \mathrm{MeV}\end{array}$ & $E>0 \mathrm{MeV}$ & $E>0.1 \mathrm{MeV}$ & $\begin{array}{l}\text { Lynch's } \\
\text { Regression } \\
\text { Analysis }\end{array}$ \\
\hline 1.0 & 1.0 & 0.9 & 0.7 & 1.1 & .8 & .6 \\
\hline 1.0 & 1.0 & - & 1.0 & 1.7 & 1.3 & - \\
\hline 2.1 & 0.8 & 1.8 & 5.6 & 0.6 & 2.7 & .2 \\
\hline 2.1 & 0.5 & - & 7.5 & 1.0 & 4.2 & - \\
\hline 0.6 & 0.3 & 0.3 & 58.0 & 0.4 & 43.0 & .7 \\
\hline 0.9 & 0.1 & - & 79.0 & 0.6 & 67.0 & - \\
\hline
\end{tabular}

* Note that the nonconservative results are $>1$ and are underlined.

$\star \star G^{\circ}(E)$ least squares fit to form $G^{\circ}(E)=A \cdot G(E) \cdot E^{b}+C / \sqrt{E}-A, b$ and $C$ are parameters which are fitted. The coefficients from the seven spectra study were used for $G^{\circ}(E)$ in the reduced spectral coverage case (indicated by $* \star \star)$, since the latter was found to be subject to a convergence instability when thermal data were excluded.

***Fast spectra only used in the analysis. 


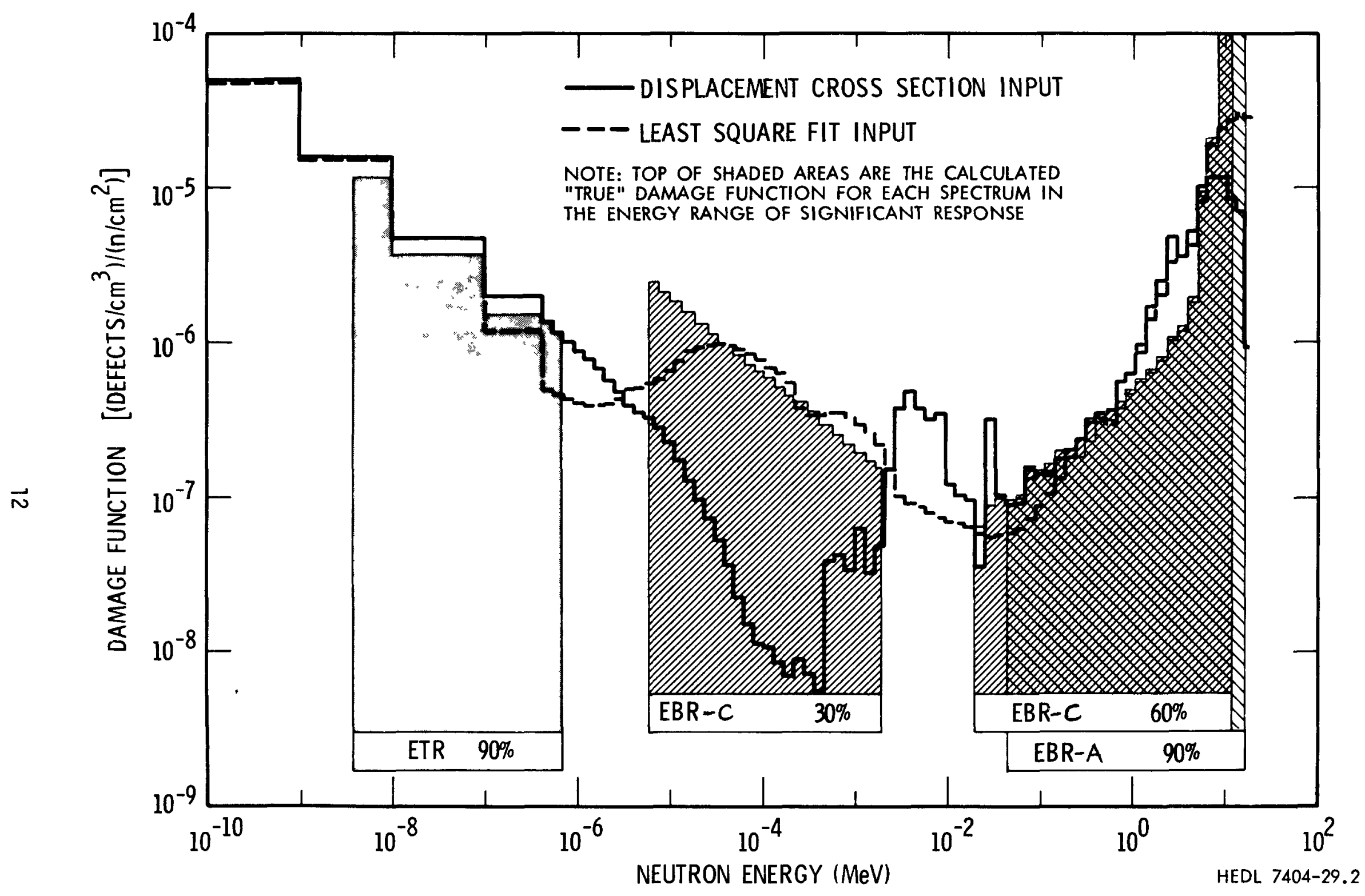

FIGURE 2. Calculated and Unfolded Effective Damage Efficiency Function for Production of $10^{16}$ Defects/ $\mathrm{cm}^{3}$ and Calculated Response Ranges for Three Test Spectra. 


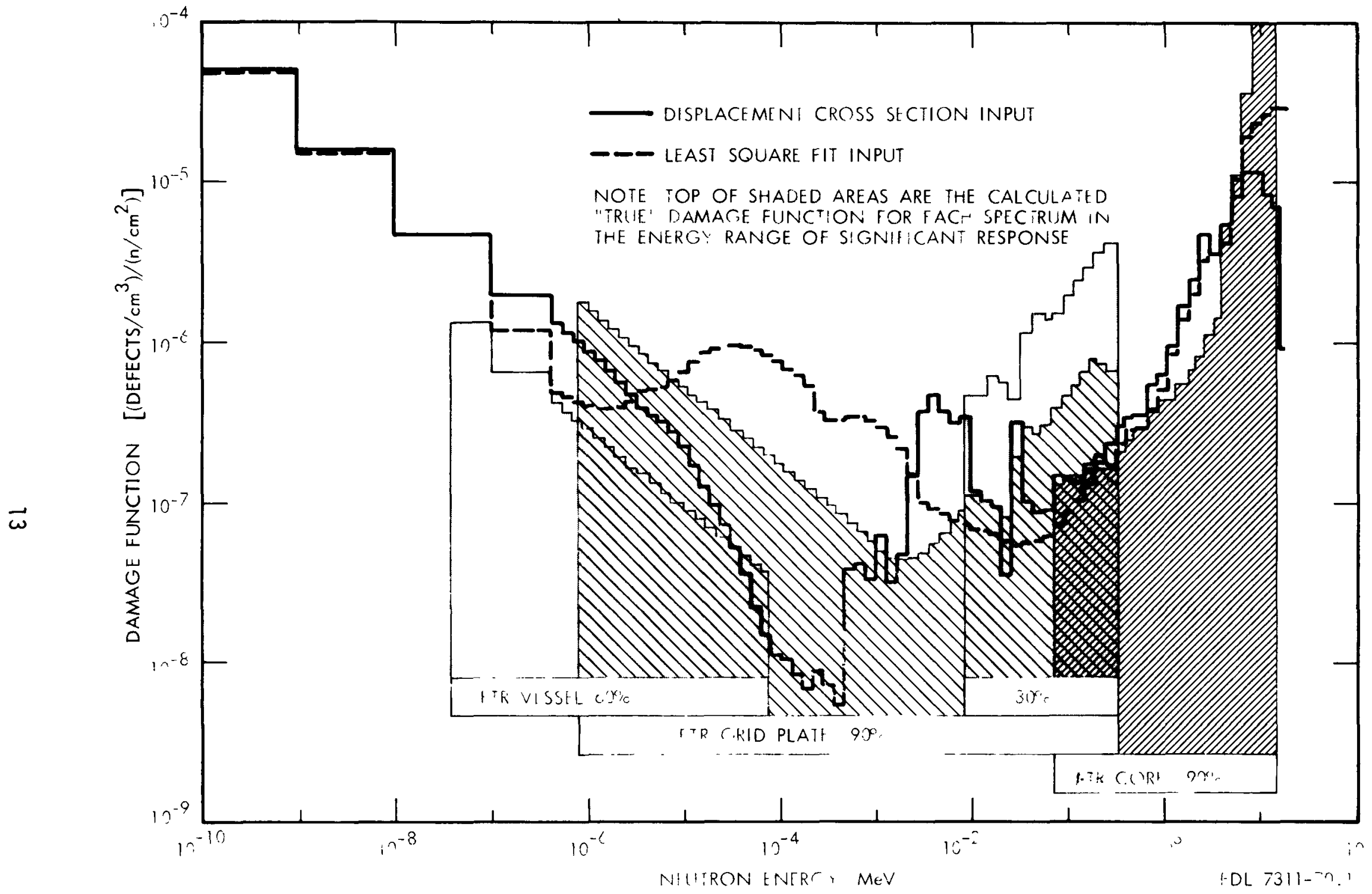

FIGURE 3. Calculated and Unfolded Effective Damage Efficiency Function for Production of $10^{16}$ Defects $/ \mathrm{cm}^{3}$ and Calculated Response Ranges for Three Design Spectra. 


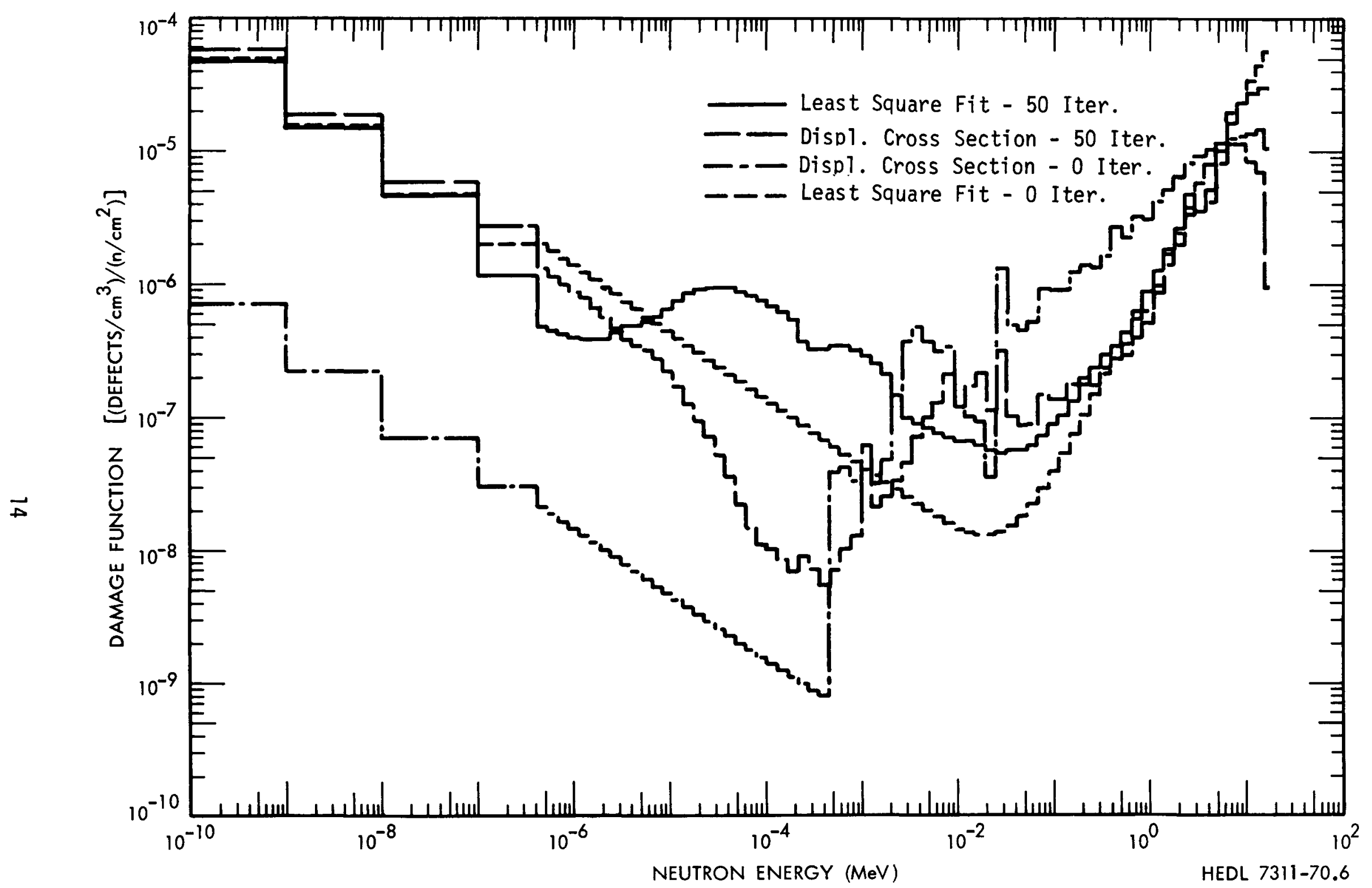

FIGURE 4. Input and Solution Effective Damage Efficiency Functions for Production of $10^{16}$ Defects/ $\mathrm{cm}^{3}$. 
ranges where an appreciable response* is generated. Figure 3 shows the calculated damage efficiency functions for the design spectra. Figure 4 shows the differences between the input $\left[G^{\circ}(E)\right]$ and output $[G(E)]$ for the two trial solution cases when seven spectra are used.

Figure 2 demonstrates that even if a damage function does not exist, as in the present case, an EDF will be defined in regions of appreciable damage response. In this case the ETR thermal reactor spectrum produced $90 \%$ of its damage from $\sim 5 \times 10^{-8}$ to $10^{-6} \mathrm{MeV}$, the intermediate spectrum EBR-C produced $30 \%$ from $10^{-5}$ to $10^{-3} \mathrm{MeV}$, and the hard spectrum EBR-A produced $90 \%$ of its damage above $\sim .1 \mathrm{MeV}$. The EDF, in the form $G_{j}^{\prime}(E)$ of Equation (3), was approximately established independently in each of these regions by the spectrum producing damage in that region. Thus the EDF is largely established from $5 \times 10^{-8}$ to $10^{-6} \mathrm{MeV}$ by the ETR spectra, from $10^{-5}$ to $10^{-3} \mathrm{MeV}$ by EBR-C and above $0.1 \mathrm{MeV}$ by EBR-A. However, this is only true if the $G^{\circ}(E)$ trial solution has sufficiently large magnitude in the energy region of interest to be subsequently adjusted in the unfolding analysis. The major significance of the magnitude of $G^{\circ}(E)$ in intermediate or low response regions (LRR's) is seen in the $10^{-5}$ to $10^{-3} \mathrm{MeV}$ energy range. Here the $G^{\circ}(E)$, based on the displacement cross section, is very low with respect to the $G_{j}^{\prime}(E)$ of EBR-C and undergoes little subsequent correction. In contrast, the least square fit based $G^{\circ}(E)$ is high enough to be fit closely to the EBR-2 form in this energy region.

*Damage response in an energy region from $E_{L}$ to $E_{U}$ is defined as

$$
\int_{E_{L}}^{E_{U}} G(E) \phi(E) d E / \int_{0}^{\infty} G(E) \phi(E) d E
$$

This is the fraction of the total damage due to neutrons in that energy region. Appreciable damage refers to any continuous energy region producing at least $20 \%$ of the damage (omitting the high and low $5 \%$ tails). We will refer to high response regions (HRR) of greater than $50 \%$, and low response regions (LRR) of less than $\sim 5 \%$ in subsequent discussions. 
Fluence limit predictions are accurate if the design spectra are generally similar to the test spectra as is seen in Table 1 and in Figure 3. The FTR core spectrum integral prediction results are nearly exact because of the similarity in the hard test spectra and this design spectrum, and the ability of the analysis to define the EDF close to the $\mathrm{G}^{\prime}(\mathrm{E})$ above $210^{-3} \mathrm{MeV}$. The thermally defined EDF at low energies is significantly larger than the FTR vessel $G^{\prime}(E)$ in the range of significant response. Thus, conservative results are predicted for the FTR vessel.

Figure 3 shows a high $G^{\prime}(E)$ response contribution in the range of 2.01-.2 MeV for the FTR vessel which is missed in the EDF. However, the dominance of the low energy end insures the conservative results. The grid plate spectrum responds significantly both in the high energy and low energy regimes. Clearly it is not well fixed by the EDF derived from the displacement trial function. However, the damage efficiency of the softest fast spectrum to which the trial solution is fitted in the least squares fit case is about 4 times larger than that of the grid plate in this low energy region. Thus, the least squares fit method produces a reasonable and conservative solution. Both effective damage functions fail to produce the response in the higher energy range called for by the interactive model for the gird plate spectrum.

No general conclusion can be drawn for the case of highly dissimilar test and design spectra. However, for this specific choice of damage model and test spectra, DFA provides a good to conservative estimate of the damaging efficiency of the dissimilar soft vessel and grid plate design spectra. Examination of Table 1 also indicates the importance of both thermal spectrum data and a reasonable choice of $G^{\circ}(E)$ to the derivation of EDF's. The influence of using fewer test spectra is shown in Figure 5. With no low energy response from the least square fit, the EDF input is simply reflective of an extrapolation of the form developed in the lower end of the EBR-2 response regions. Since the EDF is high in this region, the results are even more conservative than with thermal spectra included for the least square fit case. The EDF from the displacement function input is not conservative, being mainly determined by the low value of the input displacement function. 


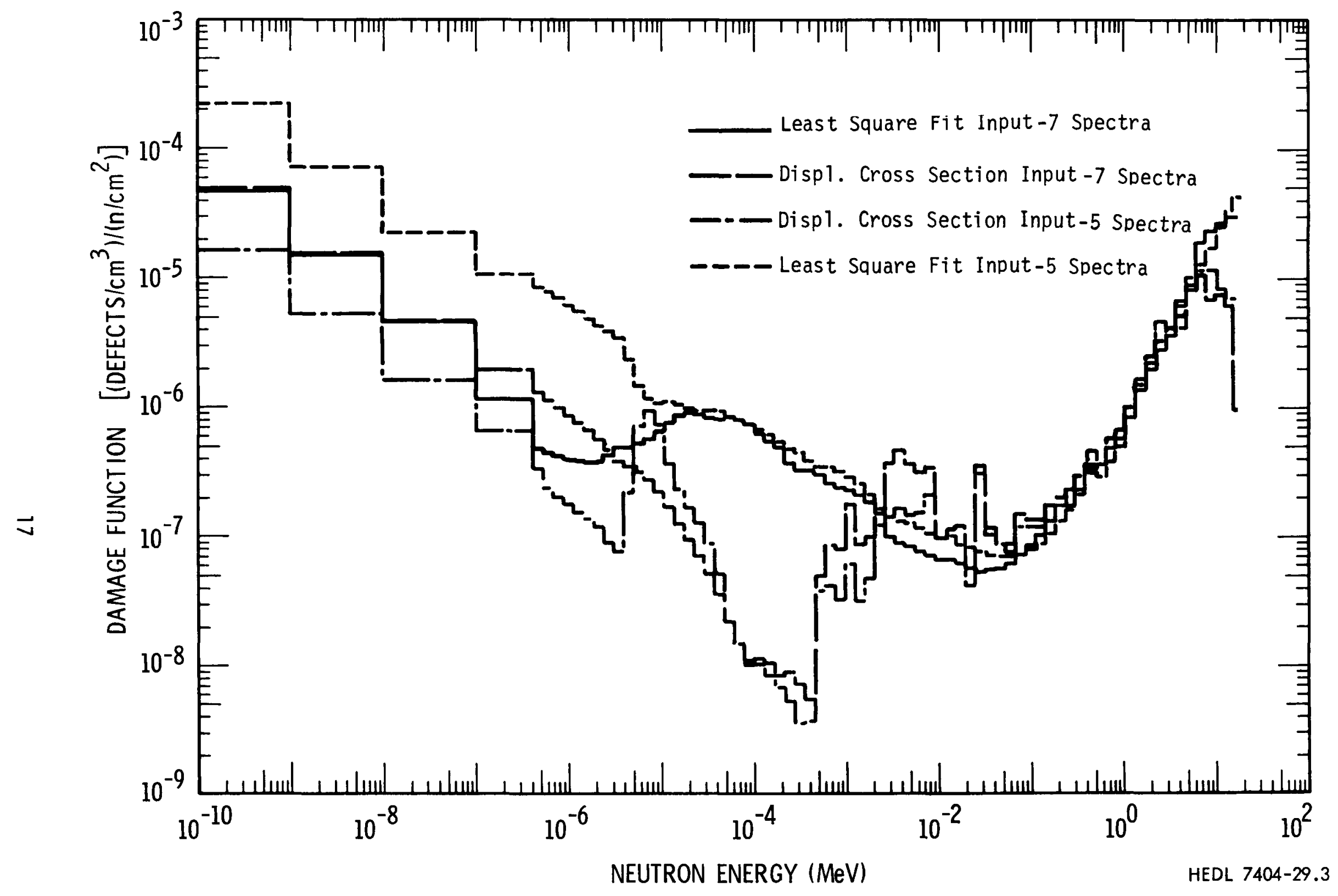

FIGURE 5. Solution Damage Functions to Produce $10^{16}$ Defects $/ \mathrm{cm}^{3}$ Using Reduced Spectral Coverage. 
Evidence of the inadequacy of the displacement function input trial function was shown by the initial disagreement between calculated and true $M_{i}$ of $\sim 130 \%$ (one standard deviation) compared to about $\sim 33 \%$ for the least square fit function. This would, in practice, indicate the inappropriateness of this form of input trial solution.

Finally and significantly, the substantial failure of other correlation procedures shown in Table 1 (mean neutron energy, total fluence, fluence $>0.1 \mathrm{MeV}$ and Lynch's method) is clearly evident.

This exercise has demonstrated that, while damage functions may not exist rigorously in all situations of interest, DFA may still be appropriate. Experimental evidence for this is the common observation of a similar fluence dependence of damage in various spectra, and the lack of observable rate effects. Further strong support for the use of DFA is the reasonable agreement often found between EDFs and predictions of noninteractive damage models, such as displacement cross section shapes.

Further direct experimental evidence that the conditions assumed in DFA are met for certain materials, properties, and spectral and temperature ranges is useful in assessing the utility of this technique. Previous EDF studies on the yield strength of stainless steel at $480^{\circ} \mathrm{C},{ }^{(4)}$ the yield strength of iron at $80^{\circ} \mathrm{C}(5)$ and the nil-ductility transition temperature of pressure vessel steels $(11)$ appear to be based on situations appropriate to DFA. On the other hand, the apparently enhanced thermal component of the total elongation damage function over the displacement function form in stainless stee $^{(4)}$ may indicate an additional effect of helium or a synergistic effect of He and displacements. In this case, the analysis may not be rigorous, but this study suggests that use of this damage function in spectra reasonably similar to those in which it was derived is nevertheless appropriate. However, extensive extrapolation between spectral shapes should not be done without further justification.

of course, there are other approaches to correlating irradiation effects data which might be more appropriate in such cases. For example, a modification of Lynch's ${ }^{(2)}$ multiple parameter correlation procedure to 
to attempt to account for interactive phenomena could be useful. Obviously one major drawback to his procedure is that there is no explicit physical information in either the activation fluence parameters or the polynominal correlation function. However a generalization of this process using nonlinear least squares analysis with more physically meaningful variables might be applied. Appropriate variables might be total, or rate of helium and displacement damage generation based on spectral information and experimental or analytical cross sections. Guidance from a damage model is needed to select the correct parameters and functional form. The least squares fit technique used in this study to obtain trial solutions for DFA (see Appendix B) represents a similar attempt based on physically reasonable functional forms. It has been incorporated into a version of SAND-II. 
$\bullet$

. 
IV ANALYTICAL MEHTODS AND DATA TREATMENT

A. SAND-II Algorithm

Since the details of the SAND-II procedure have been extensively documented elsewhere ${ }^{(17)}$, the present discussion will be limited to those points particularly germane to damage function analysis.

Basically, the procedure involves iterative perturbation of an initial input trial solution approximation in directions such that predicted integral responses successively agree more closely with the experimentally measured values. The iterations are stopped on solution stability or, alternately, when indicated errors are within expected limits. Use of the latter as an effective convergence criteria can be important in reducing error magnification through artificial introduction of spurious structure.

The basic set of nonhomogeous integral equations is replaced by a numerical quadrature in the SAND-II procedure,

$$
M_{i}=\frac{P}{\phi t_{i}}=\int \phi_{i}(E) G(E) d E=\sum_{j=1}^{m} \phi_{i, j} G_{j} \Delta E_{j} \quad i=1, n
$$

where $\phi_{i, j}$ and $G_{j}$ are assumed to be constant for each energy interval. The iterative algorithm can be written as

$$
\begin{aligned}
G_{j}(k+1) & =G_{j}^{(k)} C F_{j}(k) \\
j & =1, \ldots m
\end{aligned}
$$

where the correction factor for the $k^{\text {th }}$ iteration is

$$
C F_{j}(k)=\exp \left\{\sum_{i=1}^{n} \frac{w_{i, j}^{(k)} \ln M_{j} / M_{j}^{(k)}}{\sum_{i=1}^{n} W_{i, j}^{(k)}}\right\} \quad j=1, \ldots m
$$


and where

$$
\begin{aligned}
& W_{i, j}=M_{i, j}^{(k)} / M_{i}^{(k)} \\
& M_{i, j}^{(k)}=\phi_{i, j} G_{j}^{(k)}\left(E_{j+1}-E_{j}\right)
\end{aligned}
$$

and

$$
M_{i}^{(k)}=\sum_{j=1}^{m} M_{i, j}^{(k)} .
$$

Smoothing of the weighting function to reduce development of artificial structure can be carried out between iterations. Stating the algorithm in words, the current iterative value of the damage function in each energy interval is correctively adjusted to an amount proportional to the existing integral inconsistency weighted by the fractional contribution to the overall damage response in that interval.

Since $m>n$, the solution is obviously not mathematically unique. Thus additional information must be supplied to allow determination of a physically appropriate semi-empirical solution. This information is primarily in the form of physically based input approximations and estimates of the expected data uncertainty. Further physical insight may be gained by examining the details of the particular case being analyzed. For example, the damage response range(s) indicates something of both the range of solution appropriateness and the magnitude of the solution upper bound.

In this regard, a general observation on the nature of the damage function, gained from previous experience, can be made. There is usually a single region of appreciable response $\left(10^{-2}\right.$ to $\left.10 \mathrm{MeV}\right)$ with a secondary region at low energies $\left(10^{-8}\right.$ to $\left.10^{-6} \mathrm{MeV}\right)$ if thermal reactor irradiations are included. For the intermediate region with little response, experimental information is not presently available.

It should also be noted that the SAND-II procedure, no matter what the value of $\mathrm{m}$, has an inherently low resolution corrective ability. That 
is, finer structure might be validly applied as input information (e.g., cross sections), but cannot be accurately generated in the unfolding analysis itself. Thus, for damage functions, a quadrature of 75 lethargy groups, including 4 groups from 10 to $18 \mathrm{MeV}, 68$ groups of 1/4 lethargy from 10 to $4.4 \times 10^{-6} \mathrm{MeV}$, and 4 thermal groups is sufficiently refined. This compares with the 620 groups usually employed in spectral analysis. As in spectral analysis, group values are assumed constant and no interval averaging (fine structure flux weighting) between iterations is deemed worthwhile. Discussion of the modified SAND-II structure for damage function analysis is given in Appendix $B$.

The SAND-II procedure possesses the following characteristics, which are generally advantageous with respect to a number of other unfolding techniques:

1. Ability to incorporate a high degree of physical information in the analysis, both as trial solution and convergence criteria.

2. Physically sensible data weighting procedure based on expected contribution to spectral response.

3. Possibility of placing solution upper limits in regions of low sensitivity where approriate solutions cannot be obtained more exactly.

4. Relative insensitivity to ill-conditioning of equations and data errors as compared to techniques which rely on direct matrix inversion schemes. (This is a notorious problem in almost all such procedures.)

5. Direct numerical procedures for estimating solution uncertainties.

Disadvantages that are generally shared with other techniques include:

1. Relatively low resolution.

2. Potential for large solution errors especially in regions of low response. 
3. Solution nonuniqueness, i.e., sensitivity to trial solution selection.

\section{B. Data Extrapolation}

In order to determine the input data for damage function unfolding (i.e., fluence required to attain the desired property level), most property-fluence data must be extrapolated or interpolated to the desired property level. This has been done four different ways, depending on the data available for the analysis. These methods are discussed in the order of decreasing confidence in the results.

The most desirable procedure is to measure the total fluence dependence of damage accumulation (property change) for each spectrum. Fluence ranges must be covered which yield the property levels desired for engineering applications. In this case it is a simple matter of interpolating between data points using standard fitting procedures.

The second method is invoked when the above procedure cannot be used but when there is sufficient data from irradiation in similar spectra such that the data may be grouped according to spectrum hardness. For example, all spectra in EBR-II with mean neutron energies from 0.7 to 0.8 $\mathrm{MeV}$ could be considered as having the same damage accumulation rate. Similarly, another class could be 0.5 to $0.6 \mathrm{MeV}$. The dependence of damage on total fluence is determined for each group, and then the data for each group is extrapolated or interpolated to the desired property level. Other indices of spectrum hardness used to group data could be displacement rates, fraction of flux $>0.1 \mathrm{MeV}$, etc. However, mean neutron energy has been found to be the most useful index of those mentioned.

The third method is to correlate data with displacement rates or some other suitable damage index. The difficulty with this method is that it imposes a predetermined bias on the resulting damage function. It should be noted that displacement related damage is thought to be a dominant mechanism in many damaging environments, particularly in fast reactors. 
For extrapolation, a suitable functional form for the fluence dependence is used such as

$$
P=P_{0}+P_{S}\left(1-e^{-\beta G \phi t}\right),
$$

where $P_{0}, P_{S}$ and $\beta$ are fitted parameters. It is possible that the functional form could bias the extrapolated results. This particular form implies saturation of damage. If the functional form is not completely descriptive of the data, it will be difficult to fit the parameters.

The last method, used when only a few data points are available, is extrapolation by comparison with data of another parameter. For example, if a single data point is available at temperature $T_{2}$, and the fluence dependencies are known and are similar at $T_{1}$ and $T_{3},\left(T_{1}<T_{2}<T_{3}\right)$, then one could use the latter data to extrapolate the data for $T_{2}$. This method can introduce error due to other parameters that may not be evident with so few data points. 
$\bullet$ 


\section{TRIAL SOLUTIONS AND SOLUTION ERROR}

A. Uniqueness Uncertainty

A trial solution input, $G^{\circ}(E)$, is successively perturbed toward a solution along a path prescribed not only by the data but also the $G^{\circ}(E)$ selected. That is, the output is dependent on the input in a manner similar to the influence of the weighting function, expansion function, or trial function employed in other unfolding procedures. The SAND-II algorithm is currently the adjustment process used in DFA. (17) Solution nonuniqueness may be mitigated with physical information principally contained in the trial solution. Past experience indicates that in regions of appreciable response the damage function solution is relatively unique and independent of $G^{\circ}(E)$.

An important characteristic of the SAND-II code is a potential to estimate a conservative or high value of EDF in some regions of relatively low damage response (LRR). Such estimates can be obtained if the trial solution $G^{\circ}(E)$ has a high magnitude relative to the actual (or expected) damage function in the LRR. This generates a high to moderate response in what is actually a LRR. The result is an integral inconsistency of calculated to experimental $M_{j}$ values and the tendency of the iteration path is to lower the damage function in the LRR to remove the contradictory reponse. However, it is important to note that relatively small errors in $G(E)$ in high damage response regions (HRR) may result in large errors in LRRs. Thus generating a conservative EDF in a LRR depends on a fairly accurate and unique fit in the HRRs. The resulting conservative EDF may still be much higher than the true damage function in the LRR, but it will undergo no further adjustment due to the lack of response and the insignifiance of the remaining integral inconsistency. A low trial solution in a LRR, on the other hand, will usually undergo little adjustment. This situation should be avoided since it produces a low, nonconservative EDF in the LRR.

In the past the problem of uniqueness was treated by determining the solution envelope for a rather arbitrary set of input trial functions. This solution envelope was usually relatively narrow in HRRs, while merely 
reflecting the appropriateness of $G^{\circ}(E)$, or the the upper bound limitation discussed above, in LRRs. For displacement dominated damage, the LRR generally occurs in experimental spectra in the $10^{-6}$ to $10^{-2} \mathrm{MeV}$ energy range if thermal reactor data are included. If thermal reactor data are not used there is little damage below $10^{-2} \mathrm{MeV}$. Large uncertainties in LRRs are unavoidable if no estimates are available about the form of the solution, since there is little information in the data itself.

Input forms should reflect the general shape of theoretical predictions. Such forms include displacement, free defect, cluster, and transmutation cross sections. The use of damage models to provide physically based trial solutions is discussed in the next section. Another criterion is the initial integral consistency of $G^{\circ}(E)$ with the experimental data. If the trial solution is highly inappropriate, as indicated by the discrepancy between measured and calculated $M_{j} s$, then subsequent adjustment to an appropriate solution should be viewed as being unlikely. A reasonable rejection criterion might be that the standard deviation of calculated to experimental $M_{i} s$ is greater than the expected experimental error in the data or the standard deviation from the solution judged best. Another approach is to fit the data to an appropriate functional form, such as Equation (4). This function then serves as a starting guess for a standard interation run. Repeating the analys is while varying least squares parameters over a reasonable range gives a measure of solution uniqueness.

A computer study of uniqueness has been carried out based on $G_{D}(e)$. "True" displacement rates were calculated for the seven test spectra listed previously. These were used as data to generate solutions for a variety of input $G^{\circ}(E)$. Typical runs, shown in Figures $6 a$ and $b$, started from modified forms of $G_{D}(E)$. In both cases, the low energy $1 / \sqrt{E}$ tail was multiplied by 10 and the general slope in the high energy region $\left(\geq 10^{-4} \mathrm{MeV}\right)$ was increased for $6 a$ and decreased for $6 b$. It is seen in Figures 6a that the low energy tail and high energy region ( $>10^{-2} \mathrm{MeV}$ ) were nearly adjusted back to the true $G_{D}(E)$ form. This is because the response in the test spectra is predominately in these two energy regions. There is very little response in the intermediate energy range, and hence, the derived $G(E)$ remains similar to $G^{\circ}(E)$. 


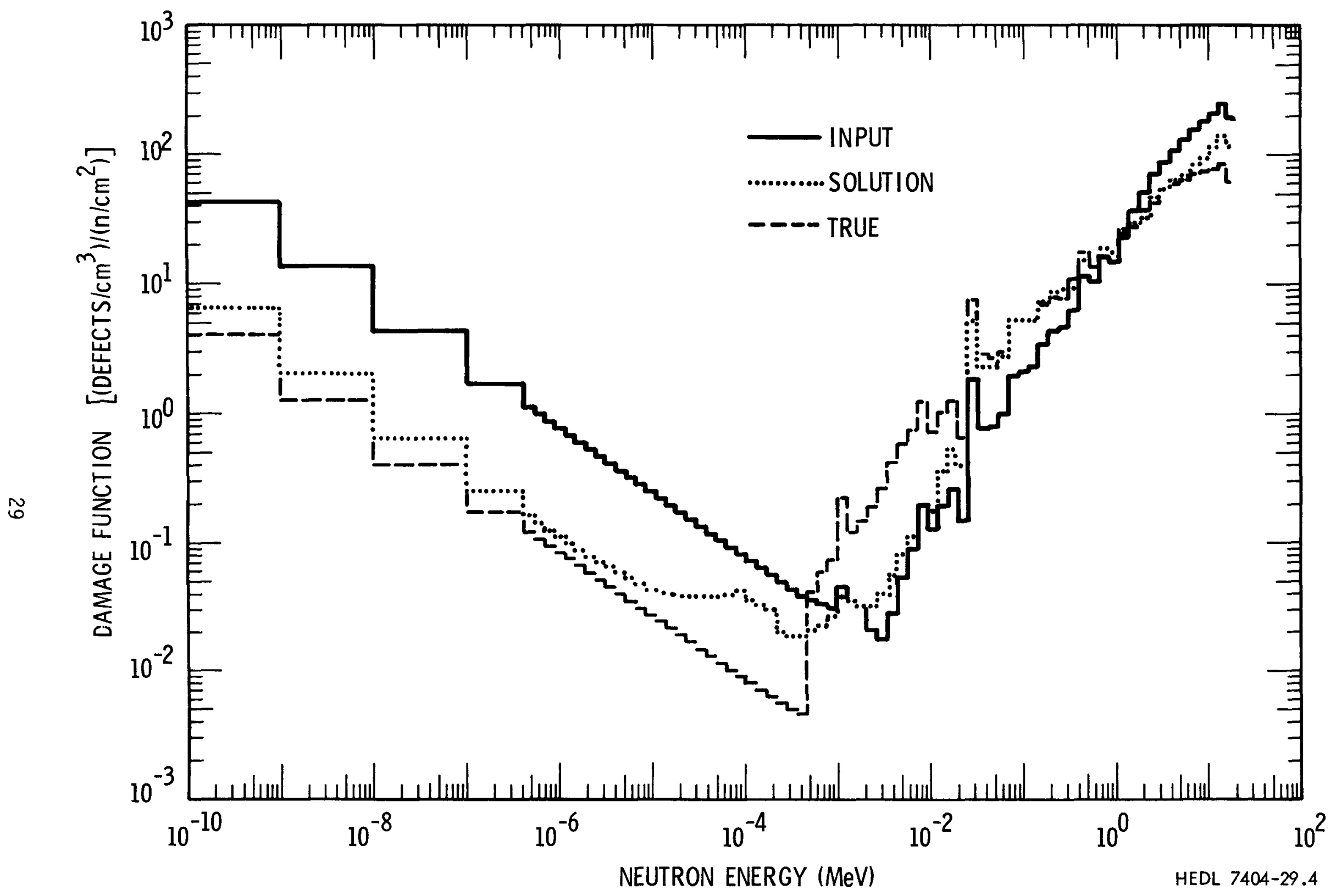

FIGURE 6a. Damage Efficiency Function Uniqueness Study, $b=+0.4$ and $c=10$. 


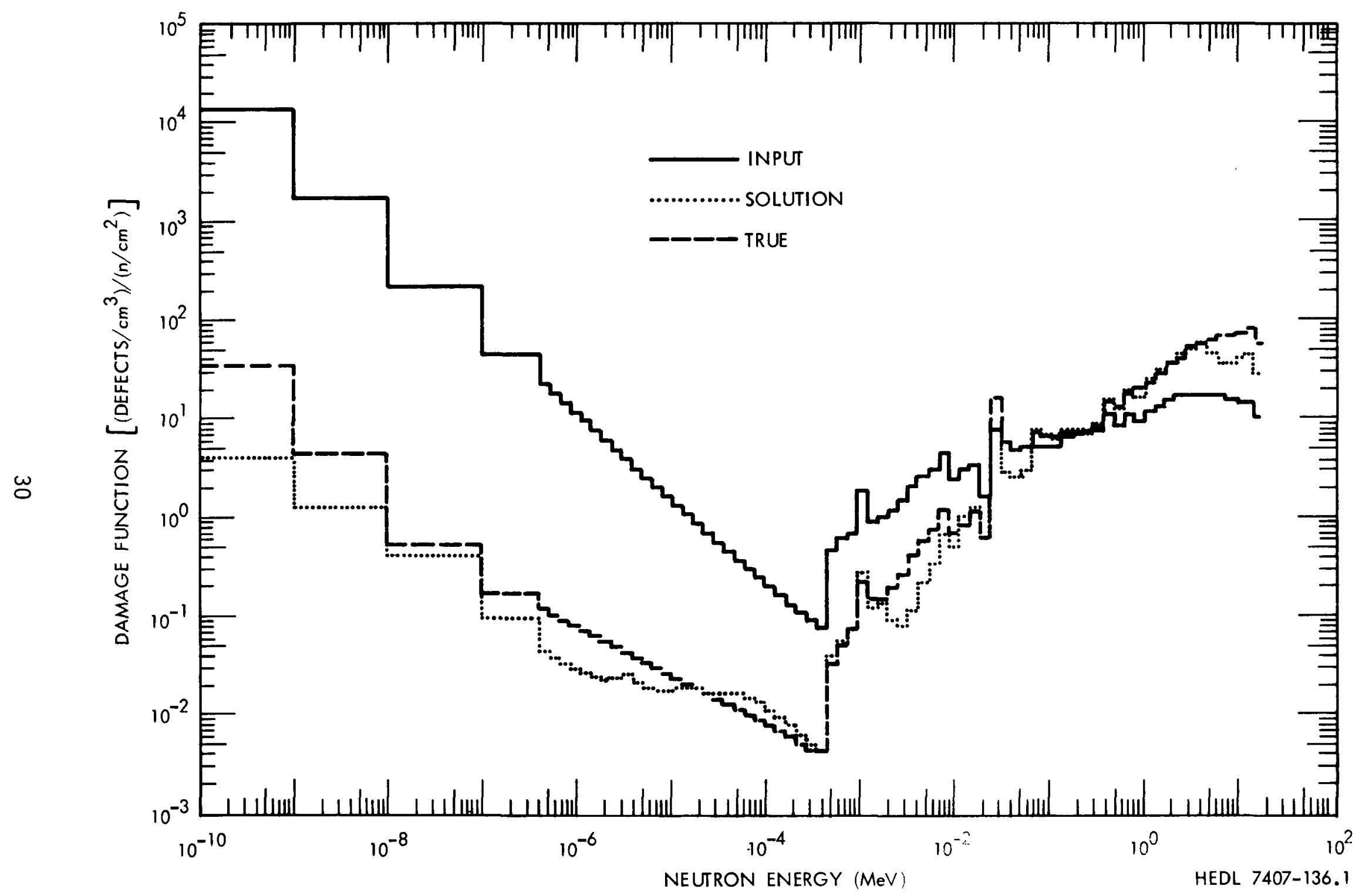

FIGURE 6b. Damage Efficiency Function Uniqueness Study, $b=0.4$ and $c=10$. 
The resulting solutions were then used to calculate fluence limits in the three FTR design spectra. The results are shown in Table 2 and Figure $7 a$ and $b$. Here $R_{I}$ and $R_{F}$ are ratios of fluence 1 imits based on input and solution damage functions respectively; i.e., $R_{I}=$ TRUE/INPUT, and $R_{F}=$ TRUE/SOLUTION. If $R_{I}$ or $R_{F}$ equals 1.0 , the input or the solution predict exactly the same fluence limit as the true fluence limit based upon the displacement cross section. If $R_{F}$ deviates from 1.0 more than $R_{I}$, then the solution is less accurate than the input.

Only in one case was the solution significantly (>1\%) less accurate, when all seven spectra were used, than the input form (least square fit solution with grid plate spectrum $R_{I}=1.00, R_{F}=1.04$ ). In the case of the core and grid plate test spectra, which are most similar to the experimental spectra, very small or large input $R_{I}$ were corrected to $R_{F}$ values of 0.89 to 1.13. For the very soft vessel spectrum, larger solution errors were encountered $\left(R_{F}=0.66\right.$ to 3.36$)$ due to the low experimental sensitivity in its dominant response range. However, the input errors in this case were even larger $\left(R_{I}=3\right.$ to 666$)$. High $G^{\circ}(E)\left(R_{I}>1\right)$ tended to produce high solution predictions $\left(R_{F}>1\right)$. In only three cases did a high $R_{I}$ give a solution prediction that was significantly (6-9\%) low.

The shape of the trial solution is very significant. For example, the constant input for which $R_{I}=63$ for the FTR vessel improved to $R_{F}=2.83$. On the other hand, a perturbed $G_{D}(E)$ input for which the low energy $1 / \sqrt{E}$ portion was increased arbitrarily by a factor of ten relative to the high energy component yielded $R_{I}=2.66$ and $R_{F}=1.00$. The solution envelope for the range of physically sensible input trial forms should represent a conservative estimate of uniqueness uncertainty. The upper bound of the solution envelope can be taken to represent a reasonable $95 \%$ confidence limit.

Accurate results can be had only if there is appreciable test spectra response in the dominant response region of the design spectra. The thermal spectra data are important in fitting the very low energy end of the response for the FTR vessel. This was demonstrated by repeating the comparison of predicted versus calculated behavior in the design spectra 
TABLE 2

SOLUTION UNIQUENESS STUDY BY COMPARING

THE FLUENCE LIMIT RATIOS TRUE/INPUT

$\left(R_{I}\right)$ AND TRUE/SOLUTION $\left(R_{F}\right)$ FOR THE FTR DESIGN SPECTRA

(Design Spectra Ratio's)

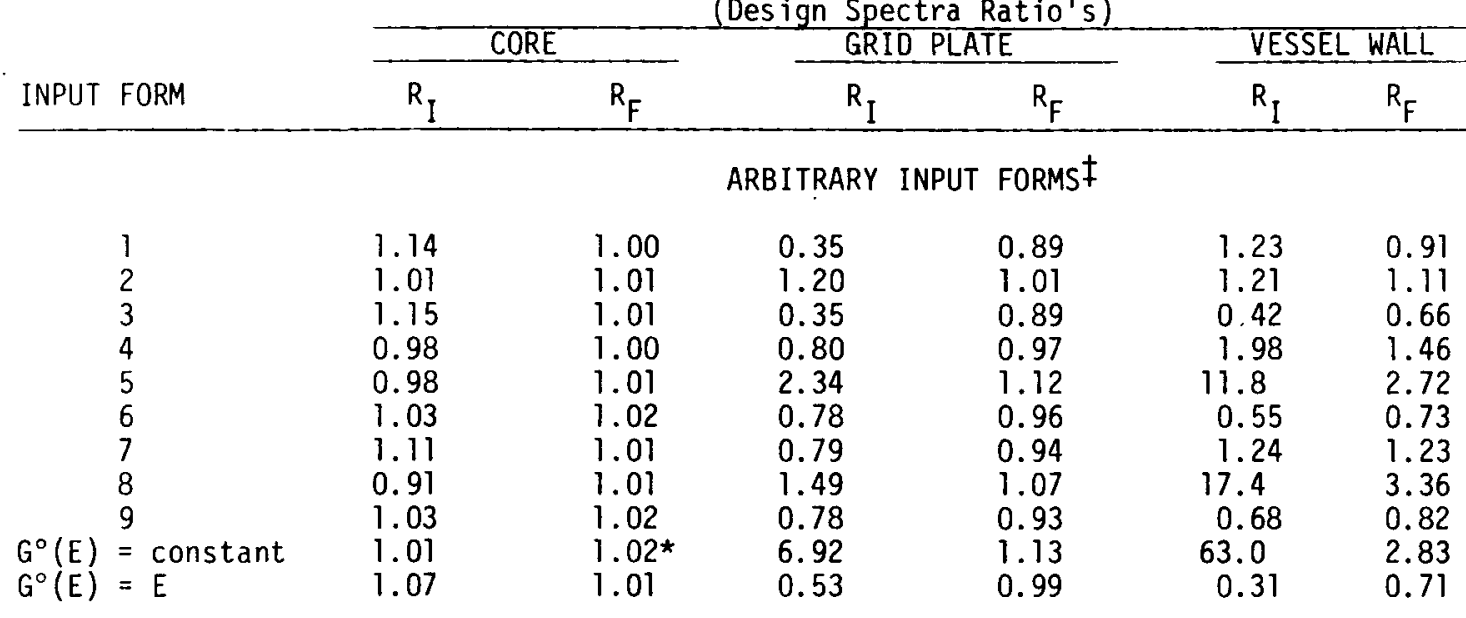

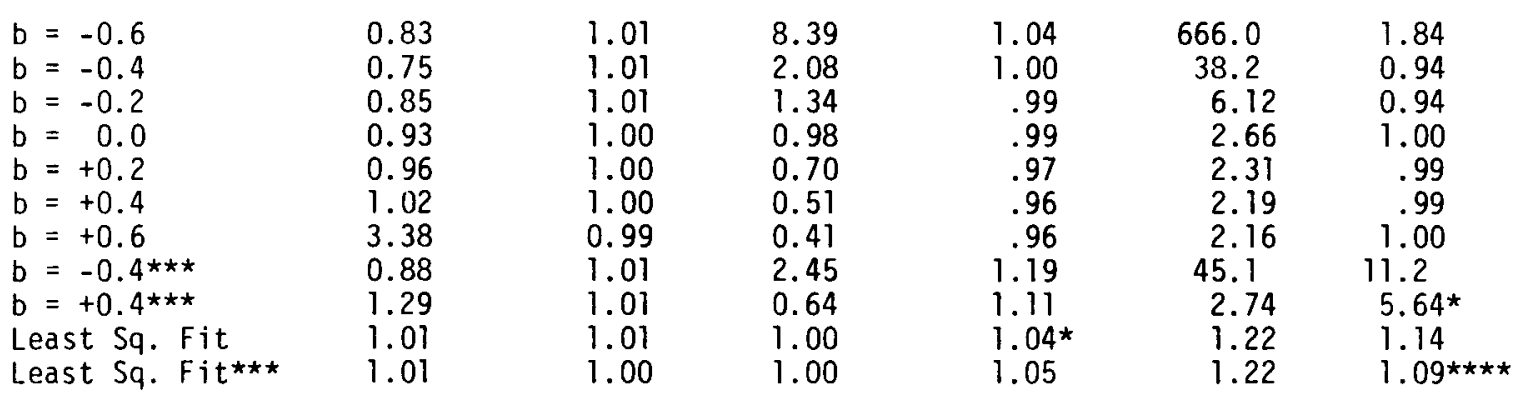

OTHER CORRELATION PROCEDURES

\begin{tabular}{|c|c|c|c|c|c|c|}
\hline $\begin{array}{l}\text { Lynch's Regres- } \\
\text { sion Analysis }\end{array}$ & -- & 0.63 & -- & 0.18 & -- & 0.72 \\
\hline$E>0.1 \mathrm{MeV}$ & -- & 1.20 & -- & 1.57 & -- & 0.71 \\
\hline$\underline{E}>0.0 \mathrm{MeV}$ & -- & 1.01 & -- & 0.14 & -- & 0.016 \\
\hline$\vec{E}(\mathrm{MeV})$ & -- & 1.07 & -- & 0.53 & -- & 0.31 \\
\hline $\mathrm{E}>0.1(\mathrm{MeV}) \star \star \star$ & -- & 0.90 & -- & 1.18 & -- & 0.53 \\
\hline$\underline{E}>0.0(\mathrm{MeV})^{\star \star \star}$ & -- & 0.89 & -- & 6.11 & -- & 55.6 \\
\hline 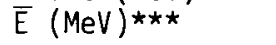 & -- & 1.24 & -- & 0.62 & -- & .36 \\
\hline
\end{tabular}

$\mp$ Combinations of piecewise continuous $G^{\circ}(E)$ segments to approximate various magnitudes of deformation of $G_{D}(E)$.

* This result shows a reduced correlation; i.e., the input gave a better prediction than the solution.

** Input $G^{\circ}(E)=G_{D}(E) E^{b}+10 / \sqrt{E}$ or least square fit to Equation 4 , where $W_{1}(E)=G_{D}(E)$ and $W_{2}(E)=1 /, \widehat{E}$.

$\star \star \star \quad$ Four EBR-II spectra only.

$\star \star \star \star$ The good fit in this case was fortuitous. Actually the least squares fit using only fast spectra was not stable and oscillated between this value and an order of magnitude larger error factor. 


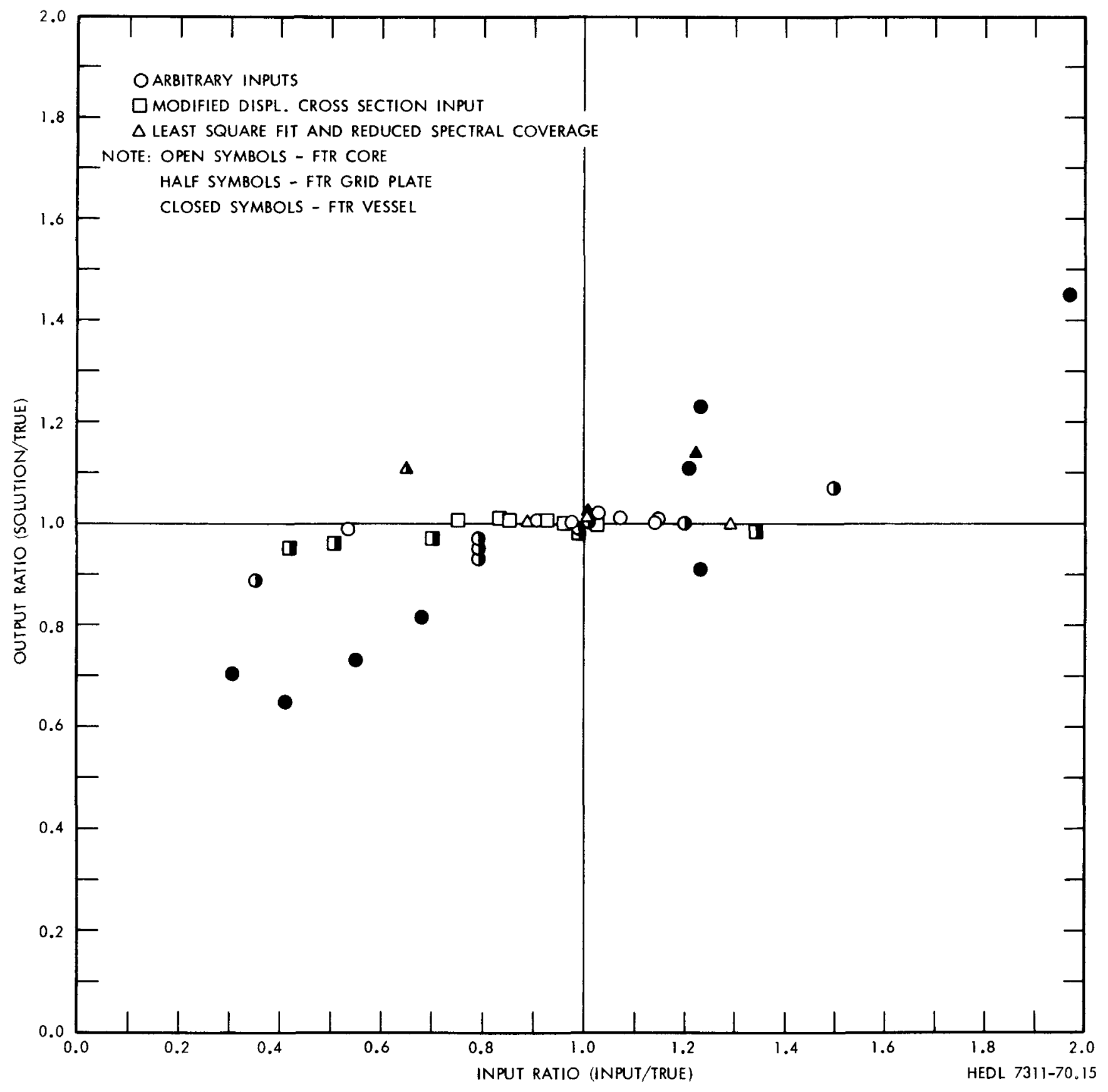

FIGURE 7a. Solution Uniqueness Study by Comparing the Fluence Limits Ratios of True/Input and True/Solution for the FTR Design Spectra. 


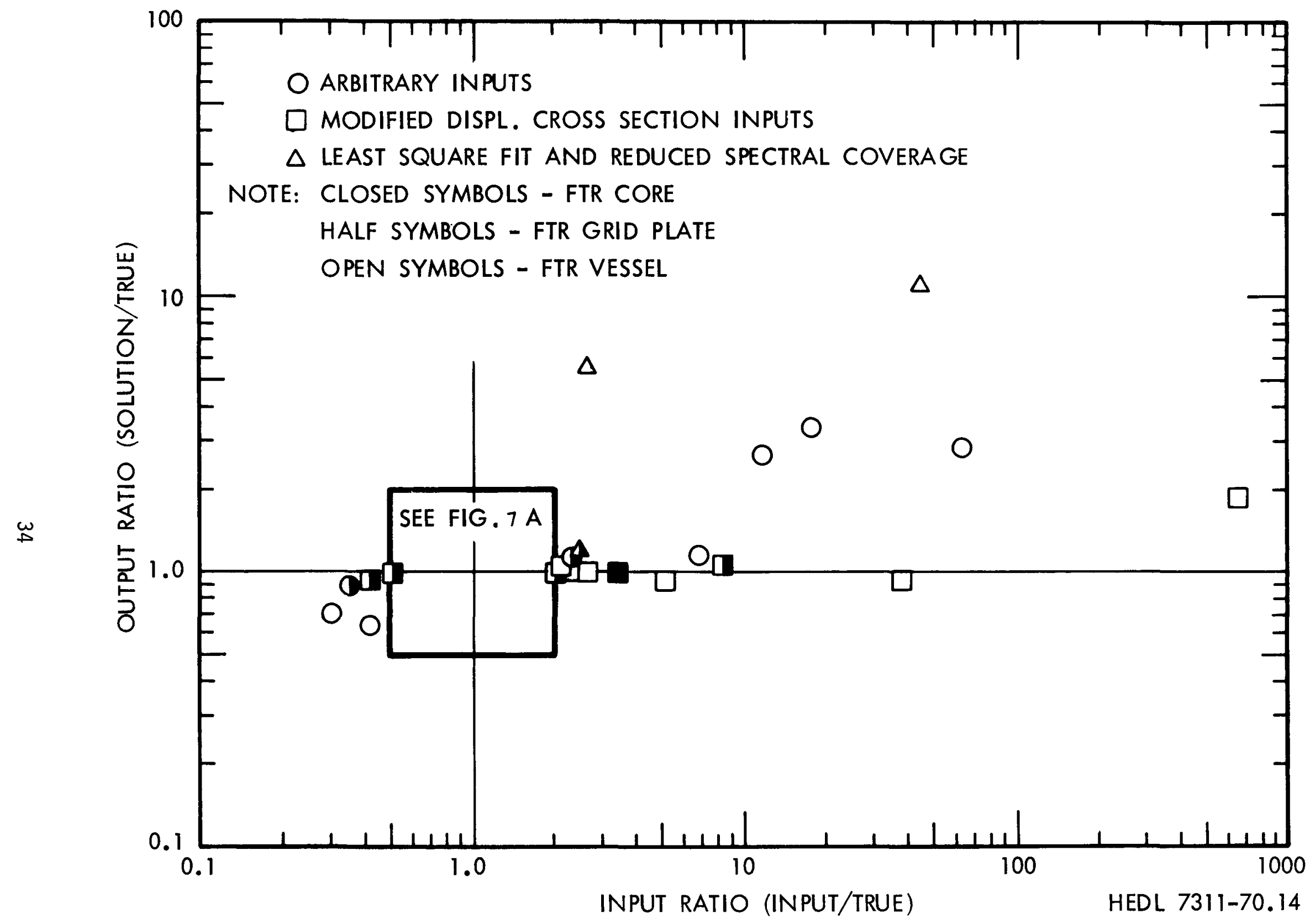

FIGURE 7b. Solution Uniqueness Study by Comparing the Fleunce Limit Ratios of True/Input and True/Solution for the FTR Design Spectra. 
using only the four EBR-II test spectra. The FTR vessel response has no test counterpart, making this the least accurately predicted displacement rate of the design spectra as shown in Table 2. Not surprisingly, a reduced correlation, from $R_{I}=2.74$ to $R_{F}=5.64$, was found for one vessel specimen case when only the fast spectra were used in the analysis.

The results obtained using other correlation procedures were generally found to be less satisfactory than the damage function approach shown in Table 2. The ratios are not too bad for the FTR core case, but as the design spectrum softens, the ratio of true to predicted deviates considerably from unity. The reasonable agreement for the FTR core is due to the similarity of the FTR core systems with the test spectra.

The results of this study of uniqueness can be summarized as follows. Only physically sensible trial solutions which are also reasonably consistent with the data should be used in DFA. In HRRs and where damage responses of a number of spectra overlap, solutions are relatively unique and independent of $G^{\circ}(E)$. In LRRs where the solution largely reflects the input trial solution, $G^{\circ}(E)$ should have a theoretical form to provide a reasonable means of interpolation or extrapolation. $G^{\circ}(E)$ should also have a magnitude in LRRs such that it is lowered on subsequent iterations. This allows estimation of the upper solution value. Thermal reactor data are needed to fix the low energy $\operatorname{EDF}\left(<10^{-3} \mathrm{MeV}\right)$ and for interpolation through the intermediate energy region $\left(10^{-6}\right.$ to $\left.10^{-3} \mathrm{MeV}\right)$. If only fast reactor data are used, extrapolation to low and intermediate energy regions $\left(<10^{-3} \mathrm{MeV}\right)$ is subject to large errors. If design spectra are generally similar to the test spectra, there should be little contribution of solution nonuniqueness to fluence limit uncertainty. Even in the case of dissimilar design spectra and inaccurate trial solutions, DFA provided substantial improvements in data correlation, and was superior to other suggested correlation procedures.

B. Damage Models and Energy Dependence

As discussed previously, trial solutions based on physical models of energy dependence are necessary to mitigate uniqueness uncertainty and to judge the appropriateness of DFA. 
Fortunately, such information about the damage energy dependence is usually available through predictions of radiation damage models. The energy dependence of damage production according to a model is generally expressed as cross sections for the production of various types of lattice defects or defect nuclei which provide a mechanism(s) for the property change of interest. In most cases, these defects will be isolated vacancies and interstitials or clusters of these point defects produced from atomic displacement cascades, transmutant gases (notably helium and hydrogen from $(n, \alpha)$ and $(n, p)$ reactions), and complexes of these defects with each other or with such impurities as carbon or nitrogen.

The damage process may be described in terms of several steps, including:

1. Generation of energetic recoiling atoms (PKA's) from a variety of neutron-nuclear interactions. It is important to specify carefully the energy spectra and density of the recoil species as the first step in damage calculations. This is probably the most tractable part of the problem.

2. Formation of some initial number and spatial distribution of displaced atoms in the displacement cascade. Among other things, partition of energy between electronic and atom motion, influence of pre-existing damage state, and the many-bodied nature of crystalline atom displacement at low energies should be included.

3. Generation of transmutant products, especially He and $\mathrm{H}$ from $(n, \alpha)$ and $(n, p)$ reactions. Location and mobility of gaseous transmutants may be an especially important factor.

4. Rearrangement of as-generated defects into stable structures (or at least metastable on an appropriate time scale). Kinetic effects should be considered.

5. The influence of this defect structure on the physical or mechanical property of interest. 
Obviously, this outlines a rather formidable task; and a detailed and complete solution is not near at hand. It might be seriously questioned by some whether the damage process in any complicated engineering material can be precisely modeled. However, it can be argued that considerably less modeling is needed when a reasonable estimate of the relative neutron energy dependence or confirmation that such an energy dependence exists is what is required. A physical rationalization of the EDF technique can be based on the following set of assumptions:

1. The production of primary defects is determined uniquely by the neutron energy. The primary defect may be, for example, displacements, free vacancies and interstitials, defect clusters or transmutant gases. The energy dependence, of course, applies only to the given set of conditions (i.e., temperature, background damage, etc.)

2. Qualitatively, the evolution of damage is unique, and the average damage efficiency per neutron determines the scale factor. For example, if the average damage efficiency in spectrum 1 is five times that of spectrum 2 then the same damage state exists when $5 \phi t_{2}=\phi t_{1}$. The properties would also be the same at this point. Relative (not absolute) damage efficiency remains constant as a function of damage accumulation; thus, a similar fluence dependence is implied.

3. If a single type of defect is not dominant, the effects must linearly superimpose, and their generation must possess similar fluence dependences. First or higher order interactive effects are not significant.

4. Kinetic phenomena or rate effects are not significant. The apparent success of damage functions in correlating data for many mechanical properties is an indication of the validity of the assumptions in these situations. These properties appear to be dominated by displacement damage as indicated by a general consistency between derived damage functions and displacement 
(or cluster) formation calculations. Preliminary evidence and theoretical considerations indicate a similar situation for the growth phase of void swelling in reactor irradiations, which is thought to be determined by free defect generation at least for moderate levels of He concentration. (31) Helium bubble swelling for materials with high He levels might be expected to have a damage function similar to the He generation cross section (corrected for possible kinetic effects).

There are several types of damage function shapes for defects or processes that might be of interest. Such a list would probably include: transmutation gases, lattice energy deposition, total displaced atoms, defect clusters of given magnitudes, and free defects. A number of such analytical damage function shapes have been calculated based on various simplifying assumptions and models and several are presented in the Appendix D.

There are, however, circumstances where an unique energy response does not exist, as illustrated by the case study of He presented earlier. The existence of synergistic interactions between defects implies a nonunique energy dependence of damage in different environments. This fact and the existence of kinetic phenomena invalidate the basic assumptions in damage function analysis. Experimental evidence indicates interactive effects might be especially significant between transmutant He and displacement damage (e.g., $500-600^{\circ} \mathrm{C}$ embrittlement of stainless steel).

A reasonable qualitative description of possible first order interactions between helium and displacements has been suggested by Straalsund and Holmes. In matrix notation, one can write

$$
M^{\prime}=\left[\phi_{j}\right]\left[\alpha_{j, k}\right]\left[\phi_{k}\right]
$$

where $[\phi]$ is the flux vector and $\left[\alpha_{j, k}\right]$ a damage matrix. The important interaction terms can be qualitatively discussed if we know something about the type of damage created in various neutron energy groups. A plausible five group matrix could be defined as follows: 
Group No.

1

2

3

4

5
Energy

Very high

High

Medium

Low

Very low
Effect

Creates helium cascades, vacancies $(V)$, and interstitials (I)

Cascades, V, and I, but no helium

$V$ and $I$, no helium

Less $V$ and I, some helium

No $V$ and $I$, much helium

With this reasonable, if not quantitative, definition of nature, the damage matrix would expand to:

$$
\left[\alpha_{i j}\right]=\left|\begin{array}{lllll}
\alpha_{11}^{\alpha_{11}} & \alpha_{12} & \alpha_{13} & \alpha_{14} & \alpha_{15} \\
\alpha_{21} & \alpha_{22} & \alpha_{23} & \alpha_{24} & \alpha_{25} \\
\alpha_{31} & \alpha_{32} & \alpha_{33} & \alpha_{34} & \alpha_{35} \\
\alpha_{41} & \alpha_{42} & \alpha_{43} & \alpha_{44} & \alpha_{45} \\
\alpha_{51} & \alpha_{52} & \alpha_{53} & \alpha_{54} & \alpha_{55}
\end{array}\right|
$$

The important interaction terms, which are enclosed in boxes, are helium in group 1 interacting with displacement damage from groups 1 and 2, and low energy produced helium from groups 4 and 5 interacting with displacement damage in groups 1 and 2 . The helium generation in group 1 corresponds to threshold helium reactions and the helium generation in groups 4 and 5 correspond to helium generation by boron or the two stage nickel reaction ${ }^{58} \mathrm{Ni}(n, \gamma){ }^{59} \mathrm{Ni}(n, \alpha){ }^{56} \mathrm{Fe}$. Displacement damage is most often attributed to high and intermediate energy reaction, groups 1 and 2 . The EDF approach assumes that all off diagonal matrix elements are zero. It is possible, in principle, to semiempirically determine $\left[\alpha_{i, j}\right]$ using mehtods similar to those applied in conventional damage function analysis. Such possibilities will be investigated in future work. Nevertheless, it is important to understand that none of the available approximate methods of data correlation, including DFA, are universally valid. Substantial 
improvement in the prediction of materials irradiation response will depend on both generation of data in well defined experiments and development of generalized damage models which are capable of extending the available data base to new conditions when required. Recent efforts to go from displacement to free or residual defect parameters for data correlation (18), and modeling of synergistic He-displacement interactions $(19)$ are examples of such work. Even though modeling alone is not sufficient, it can suggest new ways and parameters to correlate and apply experimental data. 


\section{DATA ERROR PROPAGATION}

The existence of inherent data errors usually precludes determination of unique solutions to the EDF, even if direct methods are used. These errors can be grouped into three classes: (1) random, uncorrelated, and normally distributed; (2) systematic; and (3) uniform biases. The last of these can be simply accounted for by assigning some scale error to the damage function solution and will not be discussed further.

The various error sources are collected into an integral and differential contribution. Taking the integral parameter as $M_{i}=P / \Phi t_{j}$, corresponding errors $\delta M_{i}$ can be assigned. This integral error should include contributions from uncertainties in the $\Phi t_{i}, P$, extrapolation to a common property level, and any data corrections (for temperature variations, etc.). Total random experimental fluence uncertainties are determined in the flux spectral analysis using procedures given elsewhere. ${ }^{(20)}$ These generally range from about 10 to $25 \%$ in magnitude. (21) Systematic errors in $M_{i}$ and $\phi_{i}$ for particular spectra can also be assigned. Differential errors in the flux spectra $\delta \phi_{\mathfrak{j}}(E)$ are also determined. ${ }^{(20)}$ It is assumed they have uniform magnitudes in each of a relatively small number of energy ranges (5 to 20). These errors generally are in the range of 10 to $40 \%$ (21) It is assumed that there is no correlation between the errors in the different energy ranges.

Having established error parameters $\delta \phi_{j}(E)$ and $\delta M_{j}$, a numerical procedure may be used to estimate the contribution of normally distributed random data errors to uncertainties in the fluence required to cause a given property change. The technique is nearly indentical to the error analysis procedure used in the flux spectral case. ${ }^{(20)}$ A Monte Carlo technique is used to make a large number (15-45) of recycles of unfolding runs, each with a new set of $M_{i}$ and $\phi_{j}(E)$ data appropriately selected from normal distributions characterized by the standard deviations (S.D.) $\delta M_{i}$ and $\delta \phi_{i}(E)$.

The best solution determined prior to the Monte Carlo error analysis may be used as the input trial solution at each recycle and as the "base 
solution" from which subsequent solution error analys is is accomplished. The best solution refers to the solution which is most consistent with both the experimental information and theoretical understanding of the damage process. Rapid convergence, and agreement between a physically based trial solution and the EDF would be an indication of a best solution. Using the base solution as input for each recycle allows separation of the uniqueness and data error propagation contributions to total solution uncertainty. Any number of base solutions may be analyzed. Positive and negative S.D.S for the damage function at each energy interval relative to the base solution are calculated for all Monte Carlo runs.

Since the error assignment is probably conservative with respect to the relative consistency with available integral foil data, the convergence in each unfolding is usually based on an iteration limit (20 to 50) rather than an integral error criterion. The latter might be achieved only after prolonged iteration, if at a11. Sensitivity of the results to the number of iterations should be examined. However, expected error magnitude is important physical information which should be included in the analysis if available. Artificial structure with no physical basis is likely to be generated if iterations are carried too far, or if solution error convergence criteria are set unrealistically small.

A computer study of the Monte Carlo error procedure was carried out in a similar fashion to the uniqueness study, using calculated displacement rates from $G_{D}(E)$ for the seven test spectra as input data and $G^{\circ}(E)=G_{D}(E)$. The three design spectra were used along with the EDFs to calculate displacement rates for each recycle. These displacement rates were subjected to a statistical analysis to determine standard deviation error parameters. Integral errors $\left(\delta M_{j}=0\right.$ to $\left.\pm 10 \%\right)$ and differential errors $\left(\delta \phi_{j}=0\right.$ to $\left.\pm 25 \%\right)$ in 10 energy intervals and combinations of both of these were considered. The energy region boundaries for differential errors used in this study were $10^{-10}, 9.61 \times 10^{-4}, 9.11 \times 10^{-3}, 1.11 \times 10^{-1} .6 .39 \times 10^{-1}, 1.35,2.23$, $3.68,6.07,7.79,18(\mathrm{MeV})$. Some differential results in terms of standard deviation uncertainties are shown in Figures $8 \mathrm{a}, \mathrm{b}$ and $\mathrm{c}$. 


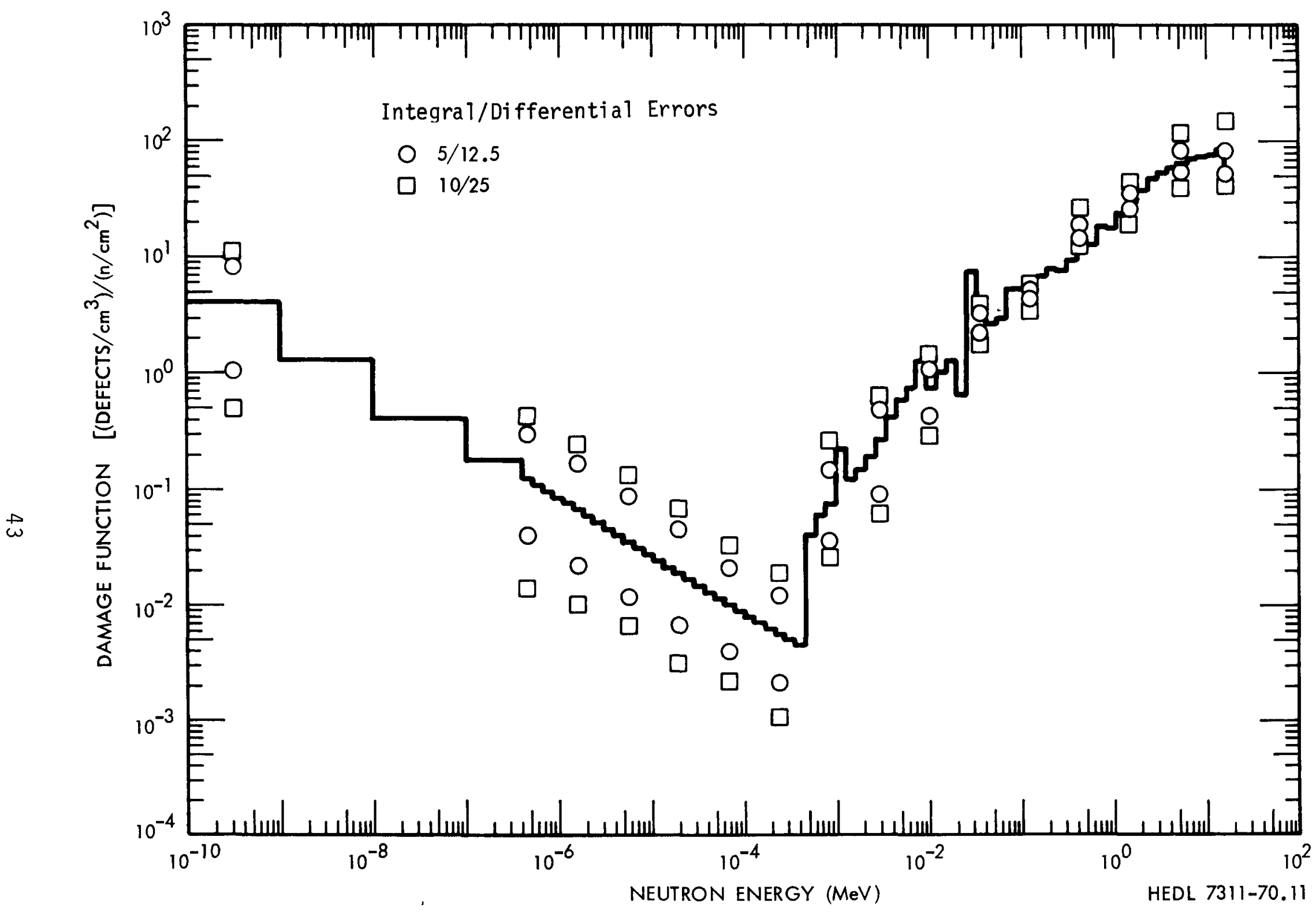

FIGURE 8a. One Standard Deviation Error Limits in the Displacement Cross Section Due to Various Combinations of Integral and Differential Errors. 


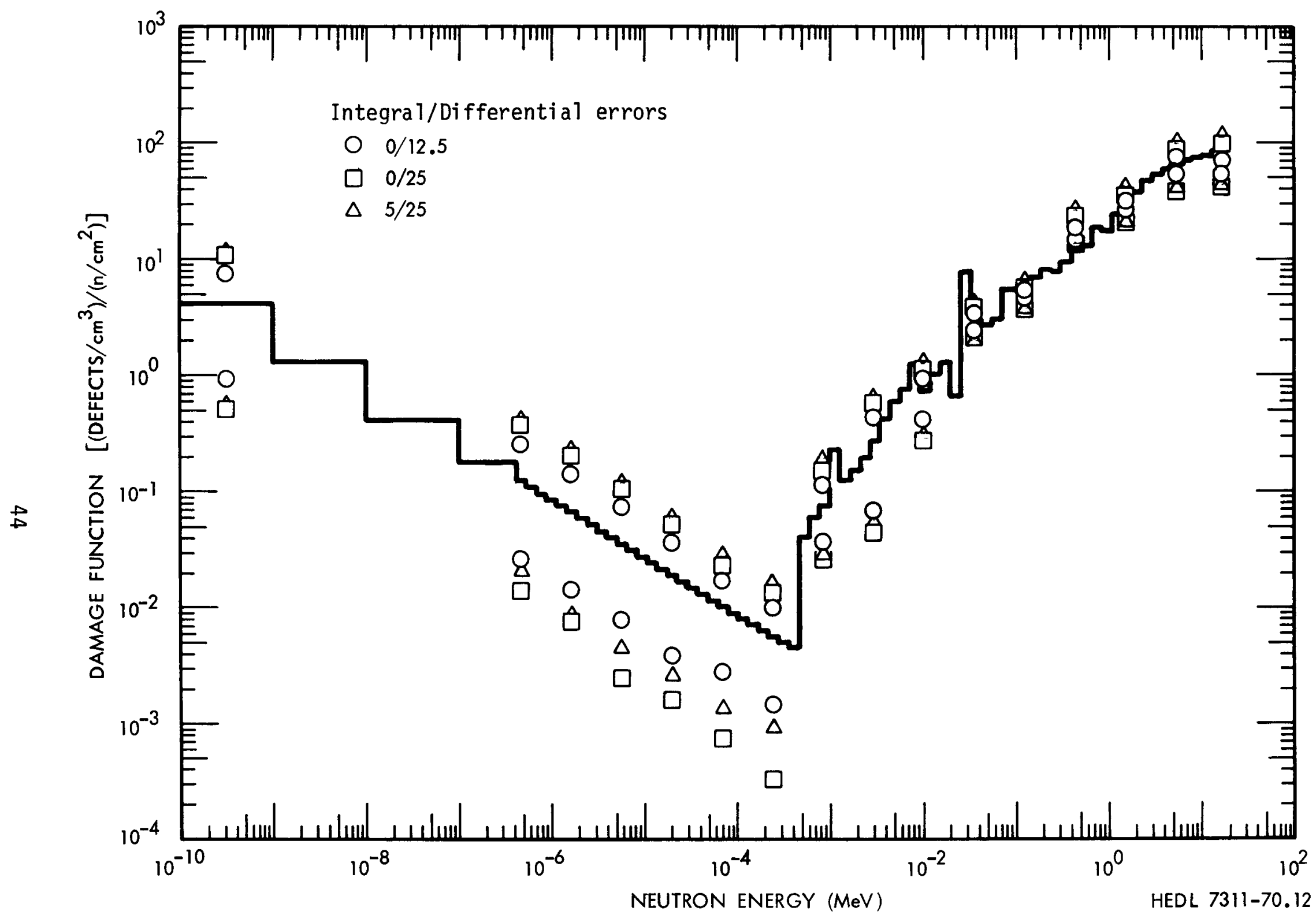

FIGURE 8b. One Standard Deviation Error Limits in the Displacement Cross Section Due to Various Combinations of Integral and Differential Errors. 


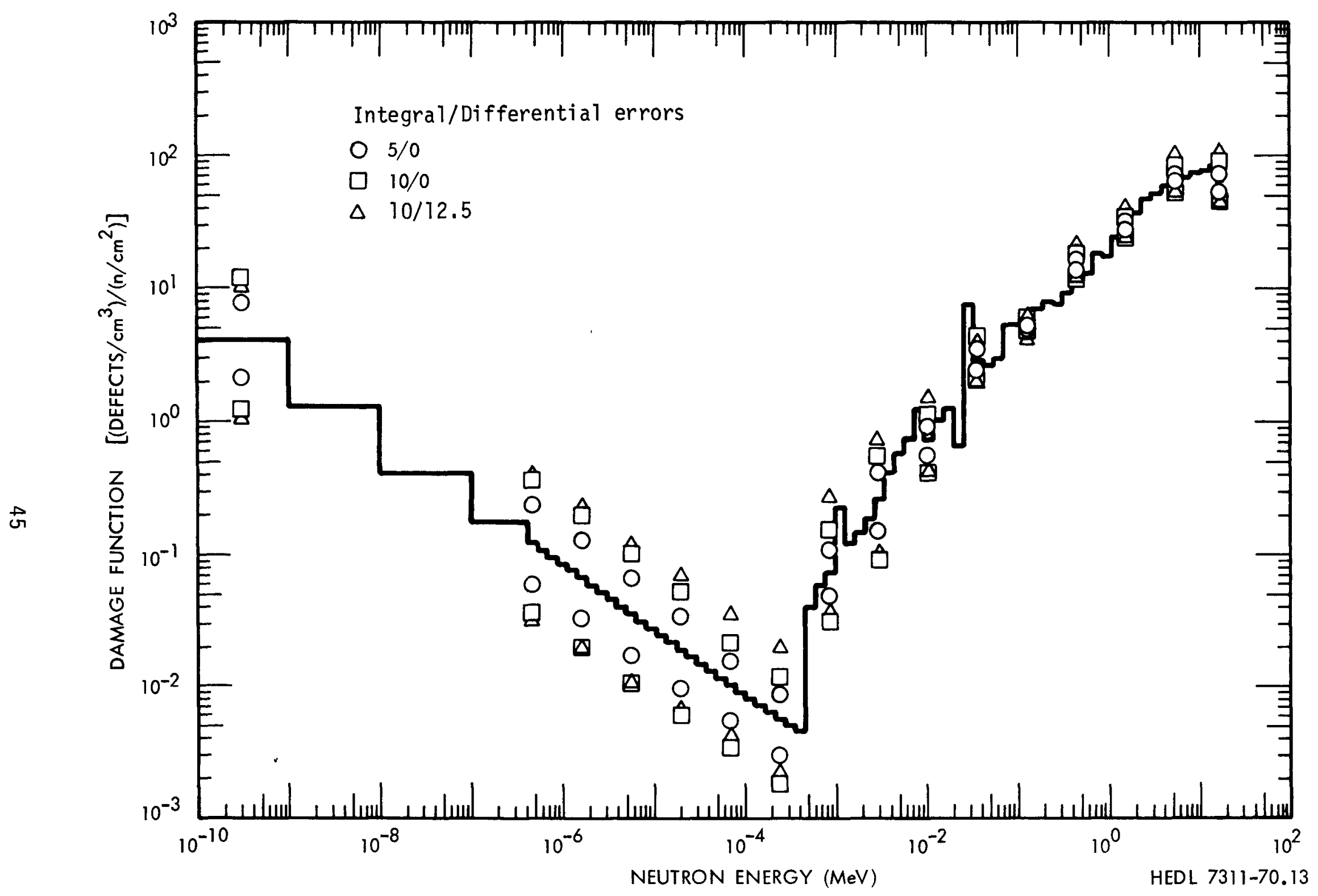

FIGURE 8c. One Standard Deviation Error Limits in the Displacement Cross Section Due to Various Combinations of Integral and Differential Errors. 
Negative predicted displacement rate S.D.s are shown in Table 3 . The largest negative predicted displacement rate errors, averaging about $40 \%$, are found in the FTR vessel spectrum which is the least similar to and has the least response overlap with the test spectra. These errors are somewhat larger than the input experimental reaction rate and spectral errors. The FTR grid plate spectrum displacement rate errors are smaller, averaging about $10 \%$, due to the greater similarity and response overlap between the design and test spectra in this case. These errors are roughly the same magnitude as the input errors. The negative errors in the FTR Core case are smal1, averaging on $1 y$ about $2 \%$. The similarity of this design spectrum to the test fast reactor spectra seems to result in random error cancellation both in determining $G(E)$ in a given energy group and group to group when $G(E) \cdot \phi(E)$ is integrated to generate displacement rates.

The displacement rate errors are surprisingly insensitive to the magnitude of the differential spectrum errors and seem to be only moderately sensitive to the integral errors. The effect of less optimal spectral coverage is also illustrated in Table 3 where the analys is is repeated using only EBR-II test spectra data for $\delta M_{i}=10$ and $\delta \phi_{i}=25$. The increase from 42 to $1800 \%$ error for the vessel spectrum displacement rate prediction again indicates the importance of thermal reactor data.

Certain types of systematic errors can contribute disproportionately to solution error. Such error magnification occurs in DFA when there is a single spectrum dominating a low to moderate response region, and its spectral shape in that region is in error. Then this energy region of the derived EDF will reflect such errors. A prime example is when thermal spectra data have large errors. To study this effect, a $G_{D}(E)$ study was carried out for cases in which only the thermal reactor displacement rate was increased and decreased by $20 \%$. The resulting solution [using $G_{D}(E)$ as an input], shown in Figure 9, is incorrect by nearly a factor of 10 in the thermal and intermediate energy regions for the positive perturbation, and a factor of 30 for the negative perturbation. This behavior is due to the fact that if the EDF is accurately fit in HRRs, the remaining integral inconsistency wi1l be removed, if required, by adjusting the EDF in LRRs. 
TABLE 3

MONTE CARLO PERCENT NEGATIVE STANDARD DEVIATIONS (\%) IN FTR DESIGN SPECTRA DISPLACEMENT RATES USING VARIOUS COMBINATIONS OF GROUP AVERAGED SPECTRAL ERRORS, $\delta \phi_{i}$, AND INTEGRAL DATA ERRORS, $\delta M_{i}$

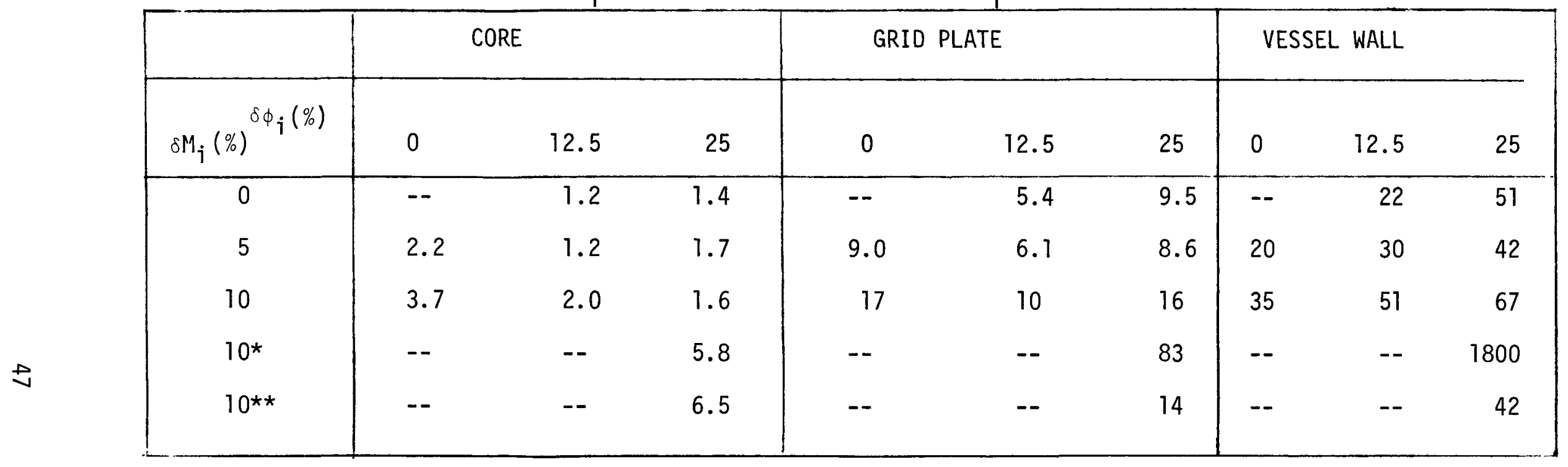

* Four EBR-II spectra only.

** Four EBR-II spectra + ETR. 


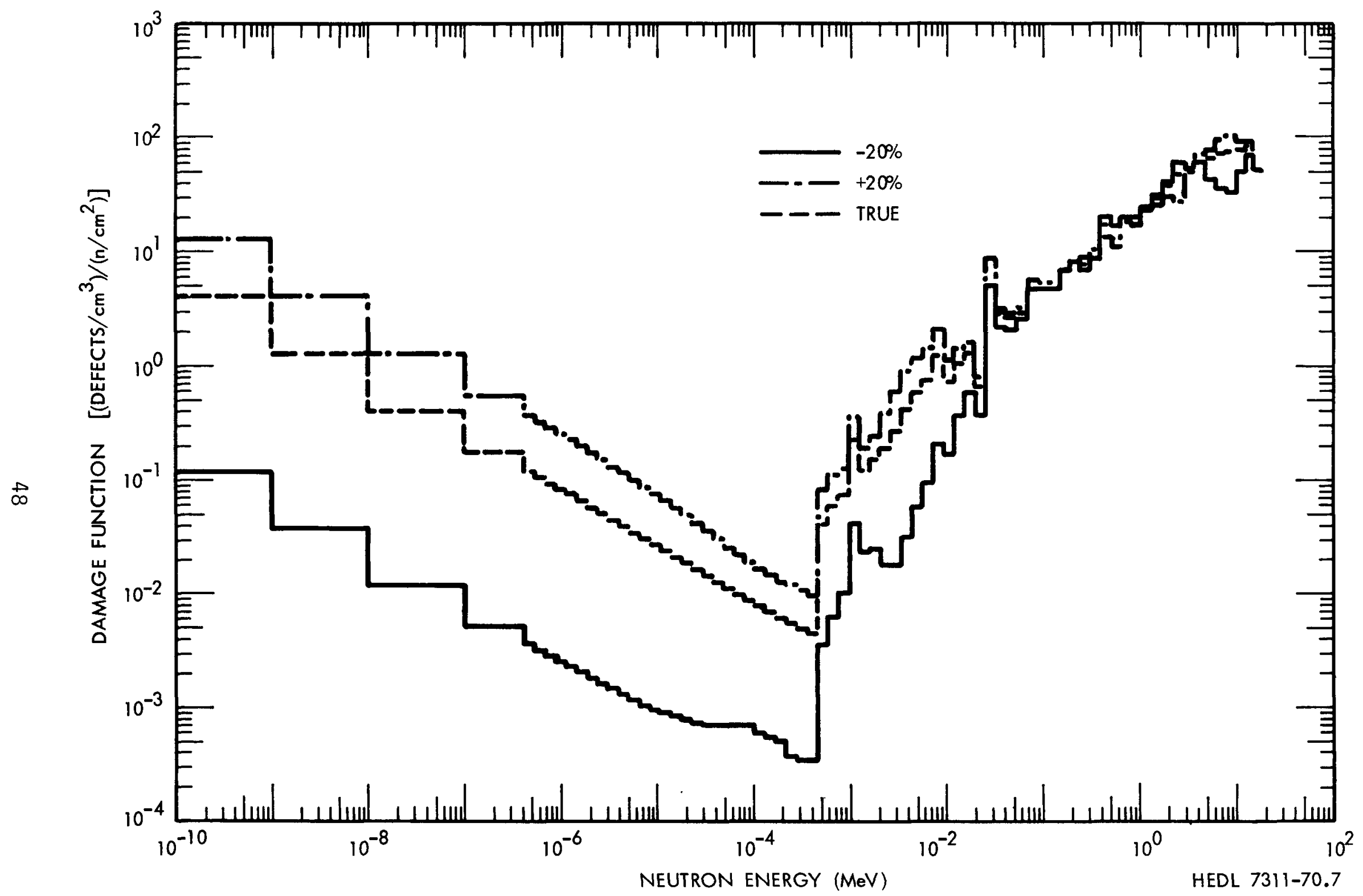

FIGURE 9. A Study of Systematic Errors by Perturbation of the Thermal Reactor. Data $\pm 20 \%$. 
For example, to el iminate a $20 \%$ error in a LRR of $5 \%$ would require a factor of four adjustment on the EDF in this region. If only a single spectrum has response in a LRR, there is nothing to prevent this behavior. However, realistic integral error convergence criteria in LRRs may mitigate this tendency to error magnification. It should again be emphasized that thermal reactor irradiation data may be the only way to establish the low energy end of the response function so that interpolation estimates of potentially important intermediate energy neutron effects in fast reactors can be made. 
-

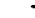




\section{TOTAL SOLUTION ERROR AND FLUENCE LIMIT ASSIGNMENT}

Having addressed, independently, damage function uncertainties due to questions of existence, uniqueness, and data error propagation, the problem remains of combining these into a total solution uncertainty and finally translating this into a lower bound fluence limit.

It is not possible to treat the question of existence explicitly. The EDF approach should be valid for situations of limited spectral extrapolation. The agreement between theoretical predictions and EDF solutions, the similar fluence dependences exhibited by radiation effects obtained in different spectra, and the lack of observed rate effects tend to support the appropriateness of DFA. If interactive effects seem to be significant and large spectral extrapolation is required, DFA is more approximate.

Systematic errors also may contribute to fluence prediction uncertainty especially when LRRs are dominated by a single spectrum. The magnitude of this effect can be estimated by artificially biasing data for that spectrum. Conservative biases might be retained in the subsequent analysis to insure that lower bound fluence limits are conservative. A large degree of spectral overlap and the use of moderately high trial solutions in LRRs are useful in reducing uncertainties due to systematic errors. Correlation uncertainty due to actual data errors can be artifically reduced by prolonged iteration; this should be avoided. It results in introducing unrealistic structures into the EDF solution.

The influence of both uniqueness and random data errors on fluence limit uncertainty can be treated in a variety of ways. One simple and conservative technique which has been used is to $f i x$ an EDF upper bound by adding twice the positive S.D., as determined in the Monte Carlo analysis based on the "best" solution, to the upper bound of the uniqueness study envelope. This allows for some undetermined covariance between uniqueness and data error propagation as contrasted with the root mean square error total. The upper bound of the EDF can then be used to predict the lower bound fluence limit in the particular test spectrum along with a nominal fluence limit from the best solution. 
A more direct technique involving a combined uniqueness - Monte Carlo study appears to be superior. A set of Monte Carlo analyses is carried out for a number $(T)$ of different trial functions. Possible bases for selection of this set were discussed in the section on uniqueness. For example, the basic damage function shape might be altered by multiplying by a function of energy such as $E^{b}$ and/or adding an energy dependent factor such as $\mathrm{C} / \sqrt{\mathrm{E}}$. Here $\mathrm{b}$ and $\mathrm{C}$ would be varied over appropriate limits. This method was generally adopted and is referred to as procedure A. Another possibility is using the perturbed data on each recycle to least square fit a function such as illustrated in Equation (4) to provide a new trial solution for each recycle. This will be referred to as procedure $B$. In any case, there are a total of $T^{\cdot} M$ EDF solutions for Monte Carlo runs of $M$ recycles. Statistical analysis based on the best solution is then carried out to establish the positive and negative S.D.S in each energy group. In Figures 10a and b are shown $G_{D}(E)$ [the best (and true) solution] and the positive and negative standard deviations in $G_{D}(E)$ for the combined errors resulting from applying procedures $A$ and $B$.

The upper bound of the EDF may be assigned as twice the positive S.D. and used to set the lower fluence limit (or in the present case, the upper bound displacement rate) at the $95 \%$ confidence level. The use of the upper bound $G(E)$ to define lower fluence limits is likely to be excessively conservative because of the integral consistency required by the data. A better procedure is to integrate the $M^{*} T$ solutions over the design spectra of interest to obtain M'T fluence limits. these fluence limits can be analyzed with respect to the nominal fluence limit to establish a two S.D. lower bound fluence limit. Table 4 shows S.D.s in predicted displacement rates for the three FTR design spectra due to variations in parameters in the combined uniqueness data error study of the displacement test case. Figure 11 shows the nominal and lower bound fluence limits at the $95 \%$ confidence level found using procedure $B$.

Comparing Tables 3 and 4 , it is evident that even for substantial perturbations ( $b=0.4$ to +0.4$)$ of the trial solution (procedure A) from the true displacement damage function $G_{D}(E)$ the total integral errors are not, on the 
average, significantly increased over the case where data errors are considered alone. Procedure B (least square fit to data on each recycle) approximately doubles the data propagation error; e.g., for FTR vessel the increase is from $67 \%$ to $134 \%$ relative to procedure $A$. Thus, in situations with good spectral coverage and when reasonably good estimates for the trial solution are available (as in the case of displacement dominated damage), data errors would be expected to cause the major uncertainty in fluence limit predictions. The effect of reduced spectral coverage is also shown in Table 4, again clearly demonstrating the importance of fixing the low energy EDF using thermal irradiations to allow extrapolation to the very soft FTR vessel spectrum.

Uncertainties in the spectra to which the EDF's are applied should also be treated. If the upper bound EDF is used to set fluence limits, normal error weighting propagation procedures can be used to account for uncertainties in the design (application) spectra. If the set of M'T EDF's are used, a Monte Carlo procedure can be used to combine the design spectrum error with the EDF uncertainty. This is accomplished by using a set (S) of appropriate perturbations (i.e., according to estimated group errors and integral consistency) of the design spectrum to generate a total of S.M.T fluence limits which can be statistically analyzed with respect to the normal unperturbed fluence limit to set lower fluence limit bounds.

This work has used primarily artificial data to study the appropriateness of damage function analysis for fluence limit estimation. Actual error analyses have been carried out on data taken from some mechanical property measurements on irradiated iron ${ }^{(5)}$ and stainless steel. ${ }^{(4)}$ The techniques used in those analyses to determine the uniqueness error and to combine it with the data error were not those recommended here. Nevertheiess, the results of those analyses are presented in Appendix $E$ for reference. The procedures used are also briefly noted there. 


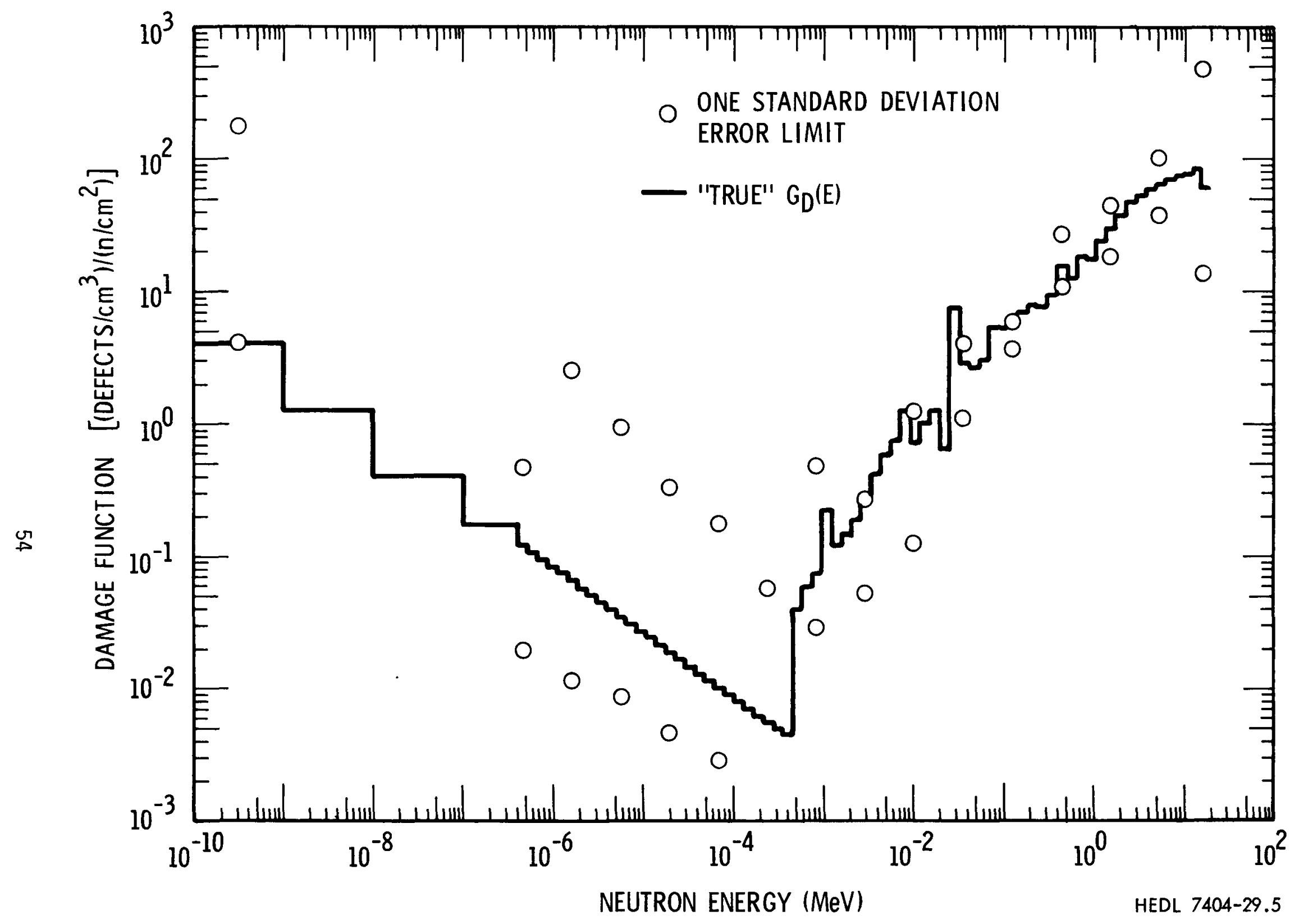

FIGURE 10a. Monte Carlo Analysis of Combined Data and Uniqueness Errors Using a Parameter Variation Approach (Procedure A). 


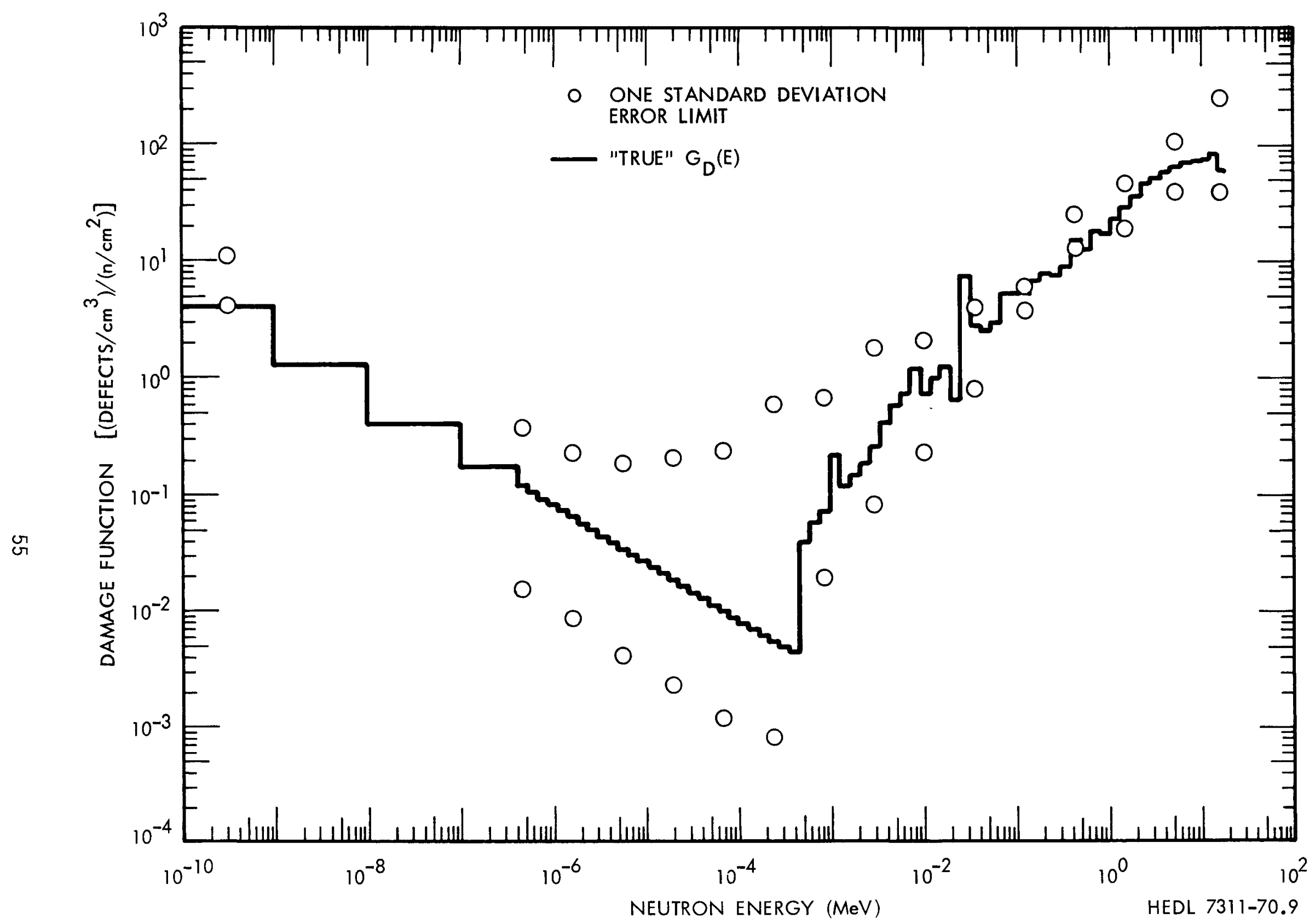

FIGURE 10b. Monte Carlo Analysis of Combined Data and Uniqueness Error Using a Least Square Fit Approach (Procedure B). 
TABLE 4

COMBINED MONTE CARLO AND UNIQUENESS PERCENT POSITIVE STANDARD DEVIATION (\%) ERRORS IN THE DISPLACEMENT RATES IN THE THREE FTR DESIGN SPECTRA

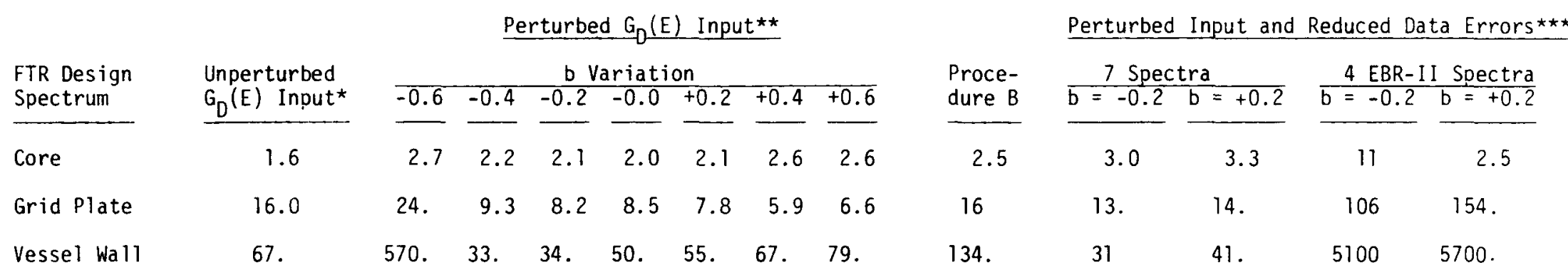

g

* Input damage function at each recycle, $G^{\circ}(E)=G_{D}(E), \delta \phi_{i}=25 \%, \delta M_{i}=10 \%$.

** Input damage function at each recycle, $G^{\circ}(E)=G_{D}(E) \cdot E^{b}+10 / \sqrt{E} ; \delta \phi_{j}=25 \%, \delta M_{j}=10 \%$, where b is varied parameter.

$\star \star \star$ Input damage function at each recycle, $G^{\circ}(E)=G_{D}(E) \cdot E^{b}+10 / \sqrt{E}, \delta \phi_{i}=12.5 \%, \delta M_{i}=5 \%$. 
MEAN NEUTRON

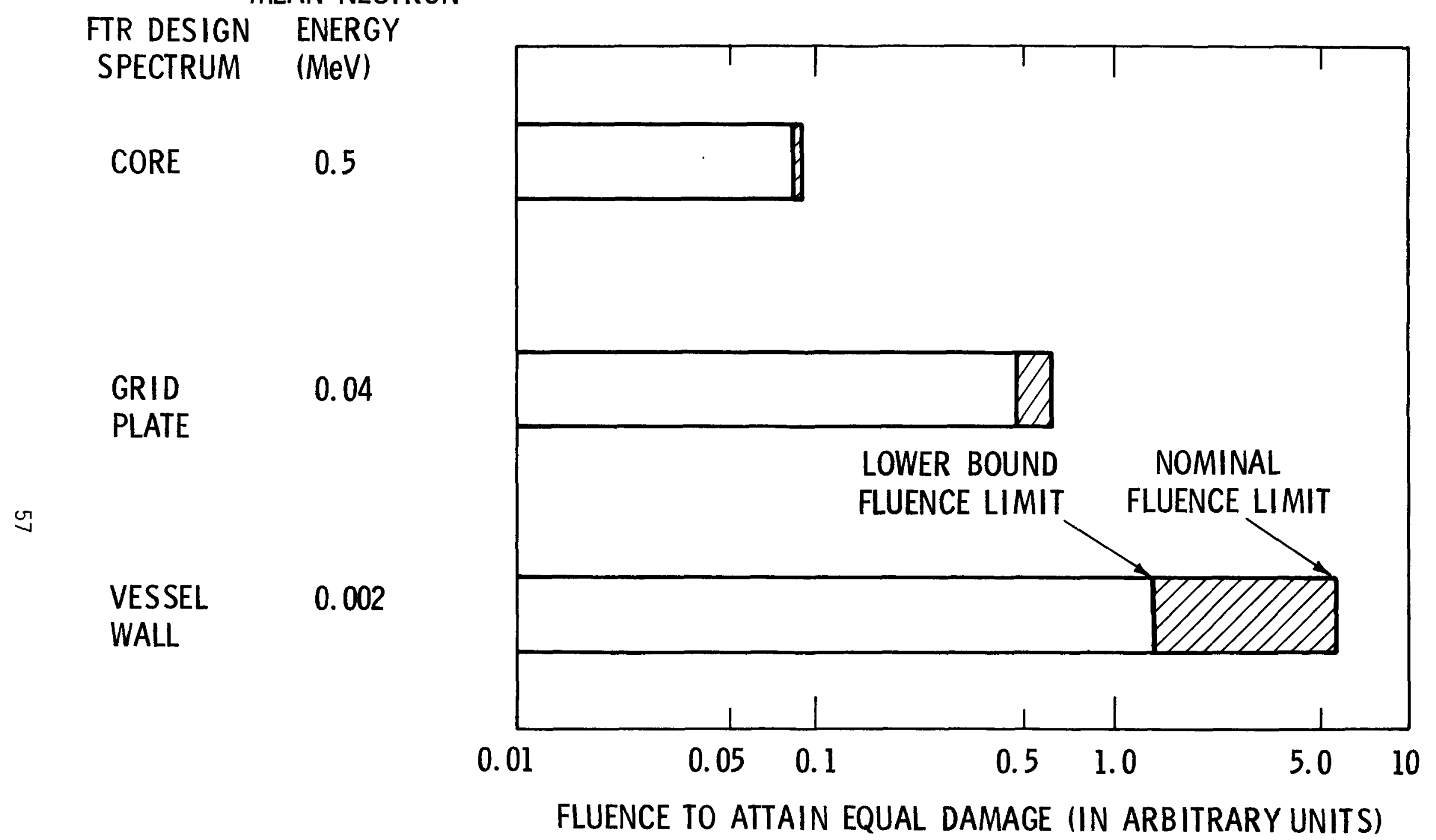

HEDL $7312-23$

FIGURE 11. Nominal and Lower Bound Fluence Limits to Attain Equal Displacement Damage in the FTR Design Spectra. 
$\bullet$

-

○ 
VIII. RECOMMENDATIONS FOR FUTURE WORK

This work has indicated several areas in which additional effort could substantially improve the correlation and application of irradiation effects data. Obviously, an extremely important one is increased accuracy of irradiation effects data, particularly in improved applied dosimetry for defining the environment. Basic dosimetry data development and testing work, such as that being accomplished by the Interlaboratory LMFBR Reaction Rate (ILRR) program ${ }^{(22)}$ in support of applied dosimetry, and use of fully instrumented fuels and materials test assemblies will be very important in working toward this end.

Another area illuminated by this work is the importance of using the widest available variation in spectra to define neutron energy effects.

Radiation damage modeling and development of basic data (e.g., He generation cross sections) are vital to reactor materials development and might be profitably accelerated. This work is needed not only for estimating spectral effects in damage function analysis, but also in analyzing other irradiation effects experiments such as charged particle simulations of neutron damage.

Finally, a generalization of damage function analysis to treat interactive effects and the exploration of using physically based multiple regression analysis techniques would be useful. 
-

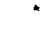

0 
IX. ACKNOWLEDGEMENTS

The authors are grateful to D. G. Doran, H. H. Yoshikawa, and

T. K. Bierlein of HEDL for helpful discussions and review of the manuscript.

G. E. Shook and N. Graves were helpful in carrying out some of the computations. The work was supported by the U. S. ERDA. 
$\bullet$

.

○ 


\section{$X . \quad$ REFERENCES}

1. Odette, G. R., "Damage Function Data Correlation for the Yield Stress of Iron," Proceedings of IAEA Specialists Meeting on Radiation Damage Units in Ferritic and Stainless Steels, Seattle, WN, Oct. 1972. Also to be published in Nucl. Eng. and Design.

2. Lynch, J. H., "Correlation of Irradiation Data Using Activation Fluences and Irradiation Temperature", Nuc. Tech., 15, 411 (1972).

3. Doran, D. G., "Neutron Displacement Cross Sections for Stainless Steel and Tantalum Based on a Lindhard Mode1", Nuc. Sci. Eng., 49-2, 130 (1972).

4. Simons, R. L., McElroy, W. N., and Blackburn, L. D., "Damage Function Analysis of Austenitic-Steel Neutron Induces Mechanical Property Change Data", Nuc. Tech., 10, 14 (1972).

5. Odette, G. R. and Ziebold, T. 0., "The Yield Stress Radiation Damage Function of Iron", Nuc. Sci. and Eng., 49-1, 72 (1972).

6. MCElroy, W. N., Dah], R. E. and Serpan, C. Z., "Damage Functions and Data Correlation", Nuc. Applic. and Tech., 7, 561 (1969).

7. McElroy, W. N., Simons, R. L., and Blackburn, L. D., "Yield Strength and Creep Strain Estimates for Irradiated Stainless Steel in Thermal and Fast Reactors," Trans. Am. Nuc1. Soc., 13, 144 (1970).

8. McElroy, W. N., Dahl, R. E., and Gilbert, E. R., Neutron Energy Dependent Damage Function for Analys is of Austenitic Steel Creep Data," Nucl. Eng. Design, 14, 319 (1970).

9. McElroy, W. N., Simons, R. L., Doran, D. G., and Straalsund, J. L., "Damage Function for Swelling in Type 304 Stainless Steel," Trans. Am. Nucl. Soc., 15, 249 (1972).

10. Yoshikawa, H. H., McElroy, W. N., and Simons, R. L., "The Determination of Damage Functions From Test Data," Proceedings of IAEA Specialists Meeting on Radiation Damage Units in Ferritic and Stainless Steels, Seattle, WN, Oct. 1972. Also to be published in Nucl. Eng. Design.

11. Serpan, Jr., C. Z., "Engineering Damage Cross Sections for Neutron Embrittlement of A302-B Pressure Vessel Stee1, "Proceedings of IAEA Specialists Meeting on Radiation Damage Units in Ferritic and Stainless Steels, Seattle, WN, Oct. 1972. Also to be published in Nucl. Eng. Design.

12. Serpan, Jr., C. Z. and McElroy, W. N., "El evated-Temperature Damage Functions for Neutron Embrittlement in Pressure Vessel Steels," Nucl. Tech., 13, 185 (1972).

13. Serpan, Jr., C. Z. and McElroy, W. N., "Damage Function Analys is of Neutron-Energy and Spectrum Effects Upon the Radiation Embrittlement of Steels," Reactor Materials Vol. II, 35-51, IAEA, Vienna (1969). 


\section{REFERENCES (Cont'd)}

14. Serpan, Jr., C. Z. and Steel, L. E., "Damaging Neutron Exposure Criteria for Evaluating the Embrittlement of Reactor Pressure Vessel Steels in Different Neutron Spectra," ASTM STP 426, Am. Society for Testing \& Materials, 594 (1967).

15. Gold, R. D., "An Iterative Unfolding Method for Response Matrices," ANL-6984 (1964).

16. Hilsenrath, J., et al., "OMNITAB - A Computer Program for Statistical and Numerical Analysis," NBS Handbook 101 (1968).

17. McElroy, W. N., Berg, S., and Crockett, "A Computer Automated Iterative Method for Neutron Flux Spectra Determined by Foil Activation," AFWL-TR-67-41, Vo1. 1-4 (1967).

18. Doran, D. G., Simons, R. L., and McElroy, W. N., "Spectral Effects on Neutron and Charged Particle Irradiations," Properties of Reactor Structural Alloys After Neutron or Charged Particle Irradiation, ASTM STP 570, 1975.

19. Odette, G. R. and Free, M. W., "Potential Effects of Interactions Between Transmutants Displaced Atoms in Controlled Thermonuclear Reactor First Walls," First ANS Topical Meeting on the Technology of Controlled Thermonuclear Fission, San Diego, California, CONF-740402-P2 485 (1974).

20. Oster, C. A., McElroy, W. N., and Marr, J. M., "A Monte Carlo Program for SAND-II Error Analysis," HEDL-TME 73-20 (1973).

21. McElroy, W. N., Jackson, J. L., Ulseth, J. A., and Simons, R. L., "EBR-II Dosimetry Test Data Analysis (Reactor Runs $31 \mathrm{E}$ and 31F)," BNWL-1402 (1970).

22. W. N. McElroy and L. S. Kellogg, "Fuels and Materials Fast Reactor Dosimetry Data Development and Testing," Nuclear Technology, 25-2, 180 (1975).

23. J. B. Scarborough, "Numerical Mathematical Analysis," John Hopkins Press, Sixth Edition, p. 545 (1966). 
APPENDIX A

A MODEL FOR HELIUM-DISPLACEMENT INTERACTION 
In order to study the validity of DFA in a situation where a unique damage function does not exist, a simplified model was postulated to describe He - displacement damage interaction. The model is not intended to accurately describe any particular physical phenomena, but it is, nevertheless, useful in determining possible roles of such interactive effects on the spectral dependence of radiation damage.

The material in this study was taken to be iron containing $7 \mathrm{ppm}^{10} \mathrm{~B}$. Spectral-averaged cross sections for He production were determined for the seven test and three design spectra and are shown in Table A-1. The energy dependent displacement cross section calculated by Doran ${ }^{(3)}$ for stainless steel was used to determine the spectral-averaged displacement cross sections, which are also shown in Table A-1. Cross sections for displacements and He generation are given in Appendix $D$ and the spectra are given in Appendix $C$.

The assumptions in the model included:

1. He is produced continuously via nuclear reactions in $\mathrm{Fe}$ and ${ }^{10} \mathrm{~B}$; the concentration increases linearly with time, with loss of mobile He to all possible sinks neglected.

2. Sma1l vacancy clusters are produced within displacement cascades at a rate of one cluster for every 1000 displaced atoms. These clusters are unstable and will rapidly dissolve into the lattice at a rate characterized by a time constant $\tau$.

3. If a diffusing He atom collides with a vacancy cluster, the cluster is stabilized and will not dissolve.

4. The number density of such stabilized clusters is the measure of the amount of radiation damage.

Using these assumptions, it is simple to derive an equation for the number density of stabilized clusters as a function of time in various spectral environments. The accumulation of lattice helium is described by

$$
\frac{d \mathrm{He}_{i}}{\mathrm{dt}}=\bar{\sigma}_{\mathrm{He}_{i}}{ }^{\mathrm{N} \phi_{i}}
$$


or

$$
\mathrm{He}_{i}(t)=\bar{\sigma}_{\mathrm{He}_{i}} \mathrm{~N} \phi_{i} \mathrm{t}
$$

where $\mathrm{He}_{i}$ is the hel ium volume density in the $i^{\text {th }}$ environment, $N$ the number of atoms $/ \mathrm{cm}^{3}, \phi_{i}$ the total flux, $\bar{\sigma}_{\mathrm{He}}$ the spectral-averaged helium generation cross section and $t$ the total time. The steady state unstabilized cluster population can be determined from

$$
\frac{d V C_{i}}{d t}=\frac{N \bar{\sigma}_{D_{i}} \phi_{i}}{1000}-\frac{V C_{i}}{\tau}=0
$$

or

$$
V C_{i}=\frac{N_{\tau}{ }^{\bar{\sigma}} D_{i}{ }^{\phi_{i}}}{1000}=\text { const., } \quad \frac{N_{\tau} \bar{\sigma}_{D_{i}{ }^{\phi}}}{1000}
$$

where $V C_{i}$ is the vacancy cluster volume density, $\bar{\sigma}_{D_{j}}$ the spectral averaged displacement cross section, and $\tau$ the cluster dissolution time constant.

The reaction rate between He and vacancy clusters to produce stabilized clusters is given as

$$
\frac{\mathrm{dC}}{\mathrm{dt}}=\frac{4 \pi R D N^{2}}{1000} \phi_{i}^{2} \tau \sigma_{\mathrm{D}_{i}} \overline{\mathrm{H}}_{\mathrm{He}_{i}} \mathrm{t}
$$

thus

$$
C_{i}(t)=\frac{2 \pi R D N^{2}}{1000} \bar{\sigma}_{D_{i}} \bar{\sigma}_{H e_{i}} \tau \phi_{i}^{2} t^{2}
$$

or

$$
C_{i}(t)=k_{i}\left(\phi_{i} t\right)^{2}
$$


where $K_{i}=\frac{2 \pi R D N 2 \bar{\sigma}}{1000} D_{i}{ }^{\bar{\sigma}} \mathrm{He}_{i}{ }^{\tau}$ and $\mathrm{R}$ is the characteristic vacancy cluster radius and $D$ is the He lattice diffusion coefficient.

The total fluence $\phi_{i} t$ needed to produce a given concentration, $C$, of stabilized clusters can be found from Equation A-7 as

$$
\phi_{i} t=\left(c / k_{i}\right)^{T / 2}
$$

Total fluences to produce $10^{16}$ stabilized clusters $/ \mathrm{cm}^{3}$ were calculated for the seven test and three design spectra and are shown in Table A-1. An arbitrary set of parameters of $D=4.5 \times 10^{-16} \mathrm{~cm}^{2} / \mathrm{sec}, \tau=1 \mathrm{sec}, \mathrm{R}=10 A^{\circ}$ and $\mathrm{N}-8.5 \times 10^{22} \mathrm{~cm}^{-3}$ were assumed in the calculations.

TABLE A-1

DATA FOR AND PREDICTIONS OF INTERACTIVE MODEL

Spectrum i

APFA III

EBR-A

EBR-B

EBR-C

EBR-D

ETR

CPS DUM

FTR CORE

FTR GRID PLATE

FTR VESSEL

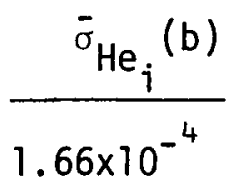

$5.02 \times 10^{-5}$

$2.33 \times 10^{-5}$

$7.35 \times 10^{-6}$

$1.24 \times 10^{-5}$

$1.23 \times 10^{-3}$

$1.24 \times 10^{-3}$

$3.12 \times 10^{-5}$

$6.83 \times 10^{-5}$

$2.64 \times 10^{-4}$

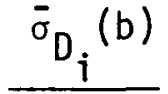

1071

654

461

279

208

358

109

434

45.7

6.95 $\phi_{j} t \times 10^{-22} \star$

.525

1.22

2.13

5.52

4.35

3.33

6.00

1.90

3.37

5.16

*For $C=10^{16}$ stabilized clusters $/ \mathrm{cm}^{3}$ 


\section{APPENDIX B}

MODIFICATION TO THE SAND-II CODE 
The damage function is determined by iteratively solving the set of integral equations

$$
M_{i}=\int_{0}^{\infty} G(E) \phi_{j}(E) d E
$$

where $\phi_{j}(E)$ is the $i^{\text {th }}$ neutron spectrum and $G(E)$ is the energy dependent damage function. The SAND-II code is used to solve the above set of equations. The SAND-II code was developed to unfold neutron spectra using reaction rate data. In order to properly account for fine resonance structure, the code was set up with 620 energy groups from $10^{-10} \mathrm{MeV}$ to $18 \mathrm{MeV}$. It is not necessary to have such a fine group structure when unfolding damage functions; consequently, a modified version of SAND-II was developed to use 80 or fewer groups. The 75 group structure presently used includes four groups above $10 \mathrm{MeV}, 1 / 4$ lethargy width groups down to $0.414 \mathrm{eV}$ and four energy groups below $0.414 \mathrm{eV}$ down to $10^{-10} \mathrm{MeV}$. With such a small number of groups, it was then possible to store the spectral data within the computer core memory as opposed to scratch drums or tapes. The combination of reduced group number and in-core storage of spectral data enabled the computer running time for unfolding runs to be reduced to $1 / 3$ that of the 620 group SAND-II. Typical running time for the 75 group structure is 240 seconds for a 50 iteration unfolding. The same modifications were incorporated into the SAND-II Monte Carlo code which is used to propagate data errors. The time savings with this code is approximately a factor of 10. Typical running times for the Monte Carlo version were reduced from 30 minutes down to 3 minutes.

A simple least square fitting subroutine was incorporated into the SAND-II code in order to provide an option for improved starting approximations to the SAND-II unfolding procedure. It is assumed that the shape of $G(E)$ is reasonably approximated by a modified displacement of helium cross section. The form chosen for $G(E)$ was

$$
G(E)=A^{2} \cdot G_{D}(E) \cdot E^{b}+B^{2} \cdot H(E)
$$


where $G_{D}(E)$ is a displacement cross section and $H(E)$ is a helium cross section. The constants $A, b, B$ are least square fit parameters. The term $E^{b}$ is incorporated to take into account the effects of annealing of point defects produced in the displacement cascade. In general, it would be expected that $b$ is small and hence the change in the displacement cross section's general slope would be small. A value of 0.2 or less might be appropriate. The constants $A$ and $B$ are squared so that no negative solutions are obtained. The absolute value of these constants are used.

The helium cross section $H(E)$ is generally taken as proportional to $1 / \sqrt{E}$. However, if the cross section producing helium were ${ }^{59} \mathrm{Ni}$, then $H(E)$ would result from a two stage reaction $\left[{ }^{58} \mathrm{Ni}(n, \gamma){ }^{59} \mathrm{Ni}(\mathrm{n}, \alpha){ }^{56} \mathrm{Fe}\right]$ with cross sections that have resonance structure superimposed on the $1 / \sqrt{E}$ form.

The least square fit technique is described by Scarborough. ${ }^{(23)}$ In symbolic form (assuming the helium cross section is of the form $1 / \sqrt{E}$ ),

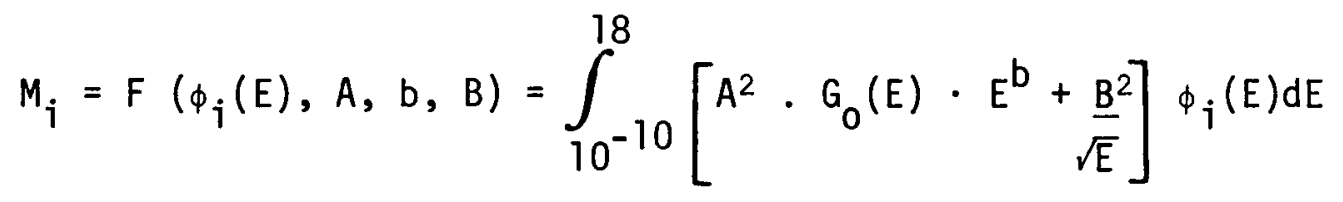

The constants are

$$
\begin{aligned}
& A=A_{0}+\alpha \\
& b=b_{0}+\beta \\
& B=B_{0}+\gamma
\end{aligned}
$$

where $A_{0}, b_{0}$, and $B_{0}$ are approximations to the constants $A, b$, and $B$ and $\alpha, \beta, \gamma$ are correction factors. The procedure is to expand the function by Taylor series and keep only first order terms to obtain,

$$
u_{i}-M_{i}=F\left({ }_{i}(E), A_{0}, b_{0}, B_{0}\right)+\left(\frac{\partial F}{\partial A}\right)_{0}+\beta\left(\frac{\partial F}{\partial b}\right)_{0}+\gamma \frac{\partial F}{\partial B_{0}}
$$


where the partial derivatives are evaluated at $\phi_{i}(E), A_{0} b_{0}, B_{0}$, and $v_{i}$ is the residual between measured and calculated values of $M_{j}$. This yields $i$ equations in $\alpha, \beta, \gamma$, which are solved by the least square method also described by Scarborough.

This procedure is applied in an iterative manner with the best fit coefficients from the previous iteration as the best guess for the current iteration. Generally 3 or 4 iterations are sufficient to obtain convergence, i.e., when the standard deviation of the residuals changes by less than $1 \%$ between iterations. If the errors in some of the data are large, the iterative procedure may nearly converge but then diverge to an unstable solution. When this condition is detected, the constants for the preceding iteration are taken as the best estimate.

The partial derivatives required for the fitting procedure are given below.

$$
\begin{aligned}
& \left(\frac{\partial F j}{\partial A}\right)_{0}=\int_{10^{-10}}^{18} 2 A_{0} \cdot G_{D}(E) \cdot E^{b_{\circ}} \phi_{j}(E) d E \\
& \left(\frac{\partial F i}{\partial b_{0}}\right)_{0}=\int_{10^{-10}}^{18} A^{2} \cdot G_{D}(E) \cdot E^{b_{\circ}} \cdot \ln (E) \cdot \phi_{j}(E) d E \\
& \left(\frac{\partial F i}{\partial B}\right)_{0}=\int_{10^{-10}}^{18} \frac{2 \cdot B_{0} \cdot \phi_{j}(E) d E}{\sqrt{E}}
\end{aligned}
$$

The residuals are the differences between measured and calculated $M_{i}$ 's and these are

$$
v_{i}=M_{i}-F\left(\phi_{j}(E), A, b, B\right)
$$

The Monte Carlo procedure used in damage analysis is the same as that used for spectral analysis, but the names of some variables or parameters have been changed to be more descriptive of damage analysis. Figure B-1 
shows a logic flow diagram for the Monte Carlo procedure. The procedure has been described in detail elsewhere. ${ }^{(20)}$ Briefly, it involves a large number of SAND-II unfolding runs yielding recycle damage functions. When the prescribed number of recycles is obtained, the set of recycle damage functions are statistically analyzed to determine the standard deviation of the recycle solution at each energy interval. Generally, at least 20-40 recycles are needed to provide adequate statistics. 


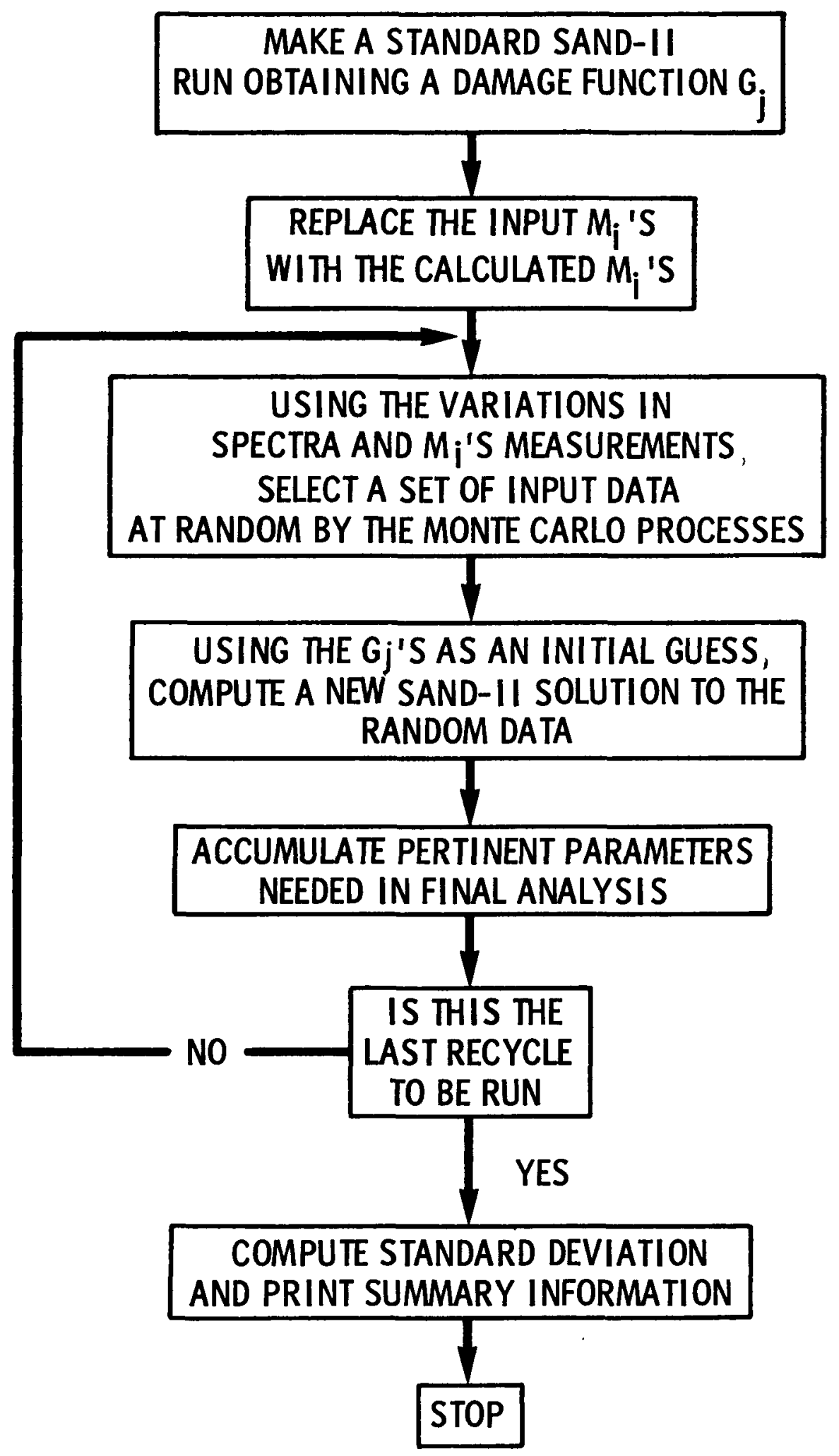

HEDL 7509-198.1

FIGURE B-1. Logic Used in the SAND-II Monte Carlo Analysis. 


\section{$\underline{\text { APPENDIX C }}$}

OVERVIEW OF THIS STUDY AND CHARACTERISTICS

OF SPECTRA EMPLOYED 


\section{Test and Experimental Spectra Information}

Damage functions are used to take into account the effect of neutron spectrum on the fluence required to attain specified property changes. They are needed because of neutron spectrum differences between irradiation facilities where data are obtained and the service position where the data are used. The damage function, which is determined by the data obtained from test reactor irradiations, and the neutron spectrum at the service location are combined to determine the fluence required to attain the specified property level. The procedure for deriving and applying damage functions is outlined by Yoshikawa, McElroy, and Simons $(10)$ and is shown in Figure $\mathrm{C}-1$. The following text serves as a guide through the figure.

1. DOSIMETRY Methods for determining the

2. SPECTRUM (the energy distribution) and FLUENCE (The total number) of neutrons during an irradiation test.

This experimental information is compared with

3. CALCULATED SPECTRUM AND FLUENCE, derived from

4. TEST REACTOR DESIGN INFORMATION, and

5. CALCULATION METHODS. The comparison of experimental and calculated spectra and fluences provide a basis for judging the adequacy of the calculation techniques. These techniques are used with information on the projected

6. REACTOR DESIGN to derive the

7. SPECTRUM AND FLUENCE expected at the point of application. In order to assess the damaging effectiveness of irradiations during application,

8. IRRADIATION EFFECTS DATA from irradiation tests, the fluences and spectra, and results from

9. DAMAGE MODELING, involving analytical studies and computer simulation, are used in the 
10. DAMAGE ANALYSIS to generate a

11. DAMAGE FUNCTION which gives the effectiveness of a neutron in causing the irradiation effect as a function of its energy. This damage function and the spectrum and fluence at the point of application are used to make a

12. DAMAGE PREDICTION. This prediction is the fluence required to cause the level of irradiation induced property change for which the damage function was derived.

The purpose of this document was to investigate the damage analysis procedure (step 10) and determine the effect of damage modeling (step 9) and errors in the data on the damage function (step 11) and damage prediction (step 12). The two damage models used in this investigation are described in the text and Appendix A. Figure $\mathrm{C}-2$ shows a schematic flow diagram of the investigation.

1. DAMAGE MODELS and

2. TEST SPECTRA were used to generate

3. ARTIFICIAL DATA. The artificial data and test spectra were input to the

4. DAMAGE FUNCTION ANALYSIS along with physically realistic

5. INPUT FORMS and

6. DATA ERRORS to generate

7. DAMAGE FUNCTIONS.

8. APPLICATION SPECTRA were combined with the damage function to

9. PREDICT INTEGRAL PERFORMANCE. The application spectra were also combined with the damage models to determine the

10. MODELED INTEGRAL PERFORMANCE. The modeled and predicted integral performances were compared to determine the

11. VALIDITY OF DAMAGE FUNCTION ANALYSIS FOR REACTOR MATERIAL FLUENCE LIMIT PREDICTIONS. 
IRRADIATION TESTS

APPLICATIONS

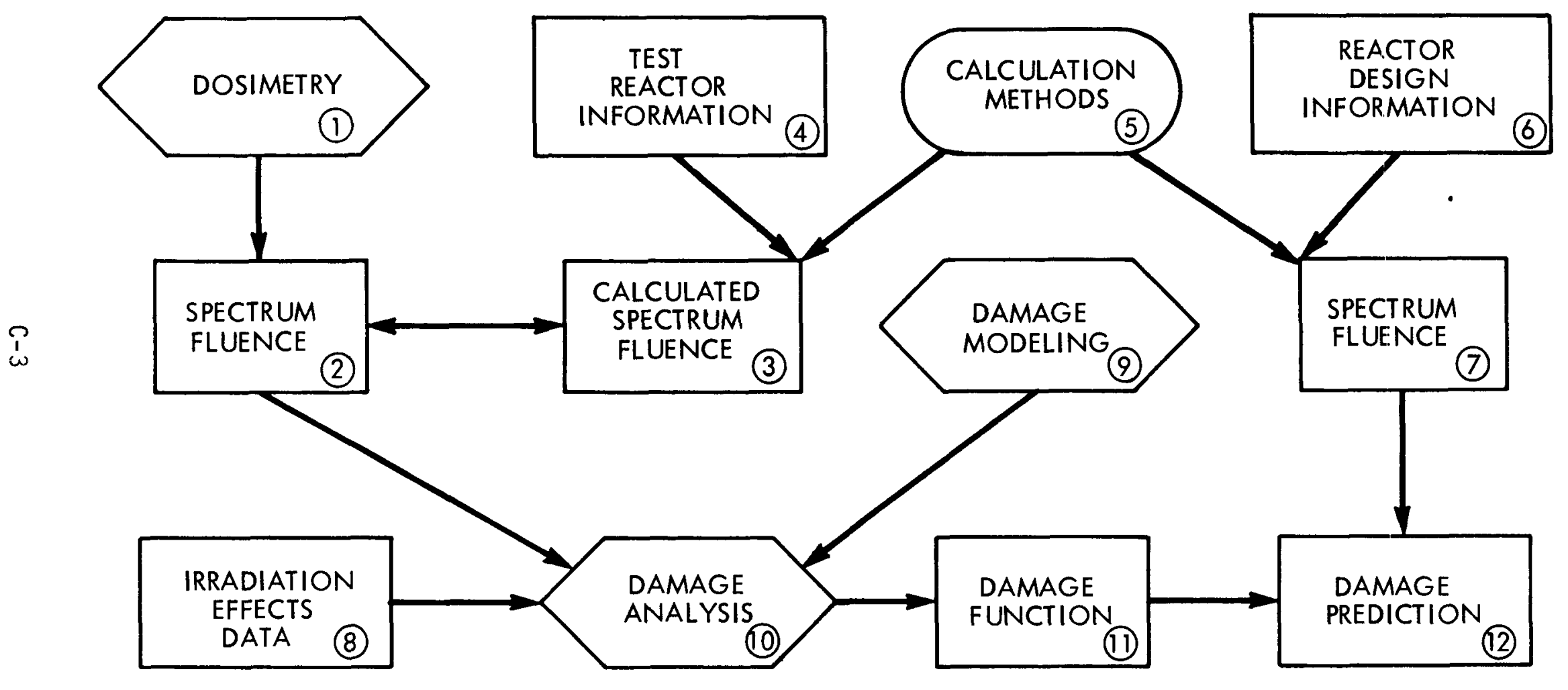

HEDL $7407-136.2$

FIGURE C-1. Goal: Predictions From Test Irradiation Data of Material Performance During Applications. 


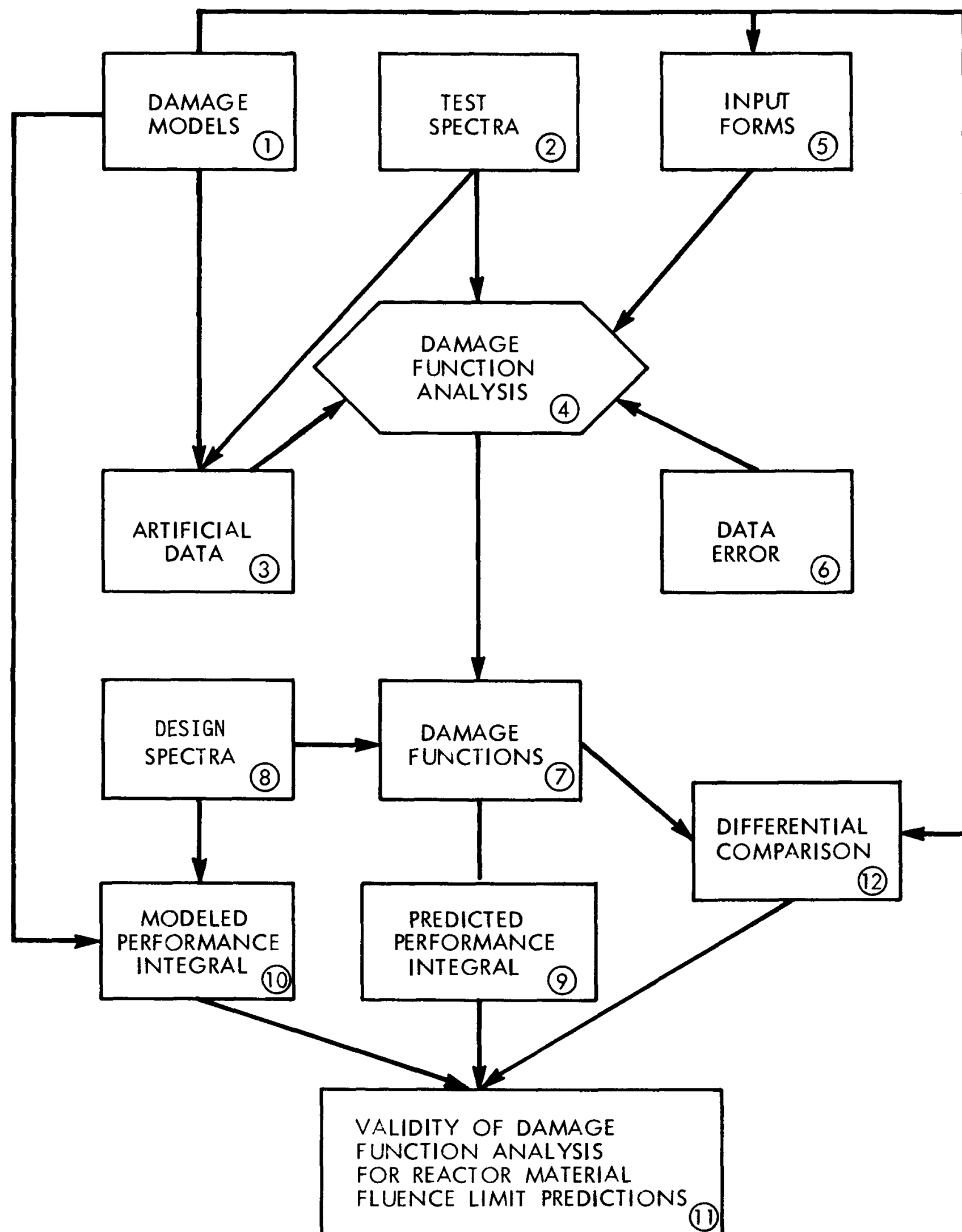

FIGURE C-2. Schematic Flow Diagram of the Investigation. HEDL 7407-136.3 
12. DIFFERENTIAL COMPARISONS were made of the damage function shapes, the physically realistic inputs and damage model predictions to determine the validity of the damage function on a differential basis.

There were seven spectra chosen to represent typical test reactor spectra used in irradiation experiments. The performance comparisons were made using three widely different, calculated FTR spectra. The ten spectra are listed in Table $\mathrm{C}-1$ along with the input data used in the computer studies and integral parameters $(\bar{E}, F>.1, \bar{D})$ which characterize the spectra.

The four EBR-II spectra represent the extremes found in the irradiation test locations in the EBR-II. The APFACC spectrum is that of a critical sphere of ${ }^{235} \mathrm{U}$. The two thermal reactor spectra, ETR and CP5 dummy, represent irradiation facilities for light water and heavy water reactors, respectively. The FTR spectra ranged from the hardest to the softest spectra found in the design of the reactor.

Figures $\mathrm{C}-3$ through $\mathrm{C}-12$ are plots of flux per unit lethargy* versus energy for the ten spectra used in the study. The figures also show the $90 \%$ response regions for displacement and helium production in Type 316 stainless steel.

*]ethargy $=\ln [10 / \mathrm{E}(\mathrm{MeV})]$. 
TABLE C-1

INTEGRAL PARAMETERS CHARACTERIZING THE SPECTRA USED IN THE COMPUTER STUDIES

\begin{tabular}{|c|c|c|c|c|}
\hline \multirow[b]{2}{*}{ Spectra } & \multicolumn{2}{|c|}{ Artificial Data } & \multirow[b]{2}{*}{$\begin{array}{c}\text { Mean Neutron } \\
\text { Energy } \\
\text { (MeV) }\end{array}$} & \multirow[b]{2}{*}{$\begin{array}{l}\text { Flux Fractio: } \\
\text { E. } 0.1 \mathrm{Me}:\end{array}$} \\
\hline & $\begin{array}{c}\text { Interactive } \\
\text { Mode] } \\
{\left[\left(\text { Defects } / \mathrm{cm}^{3}\right) /\left(\mathrm{n} / \mathrm{cm}^{2}\right)\right.}\end{array}$ & 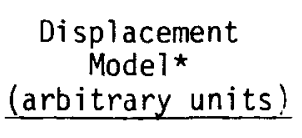 & & \\
\hline \multicolumn{5}{|l|}{ Test Spectra } \\
\hline APFACC & $1.90 \times 10^{-6}$ & 27.24 & 1.6 & .97 \\
\hline EBR-II Row 2 Midplane (EBR-A) & $0.820 \times 10^{-6}$ & 16.74 & 0.8 & .86 \\
\hline EBR-II Row 7 Midplane (EBR-B) & $0.469 \times 10^{-6}$ & 11.75 & 0.5 & .73 \\
\hline EBR-II Row $2, Z=59 \mathrm{~cm}(E B R-C)$ & $0.181 \times 10^{-6}$ & 5.66 & 0.2 & .36 \\
\hline EBR-II Row $7, z=59 \mathrm{~cm}$ (EBR-D) & $0.230 \times 10^{-6}$ & 5.31 & 0.2 & .37 \\
\hline ETR G-7 Loop & $0.300 \times 10^{-6}$ & 9.11 & 0.5 & .29 \\
\hline CP5 Dummy Fuel Element & $0.167 \times 10^{-6}$ & 2.49 & 0.1 & .099 \\
\hline \multicolumn{5}{|l|}{ Application Spectra } \\
\hline FTR Core & $0.526 \times 10^{-6}$ & 11.04 & .5 & .61 \\
\hline Grid Plate & $0.297 \times 10^{-6}$ & 1.61 & .04 & .11 \\
\hline Vessel Wall & $0.194 \times 10^{-6}$ & 0.177 & .002 & .0045 \\
\hline
\end{tabular}

*Due to a normalization error discovered late in the study, this quantity is proportional to, but not identical to, the microscopic displacement cross section for stainless steel. 


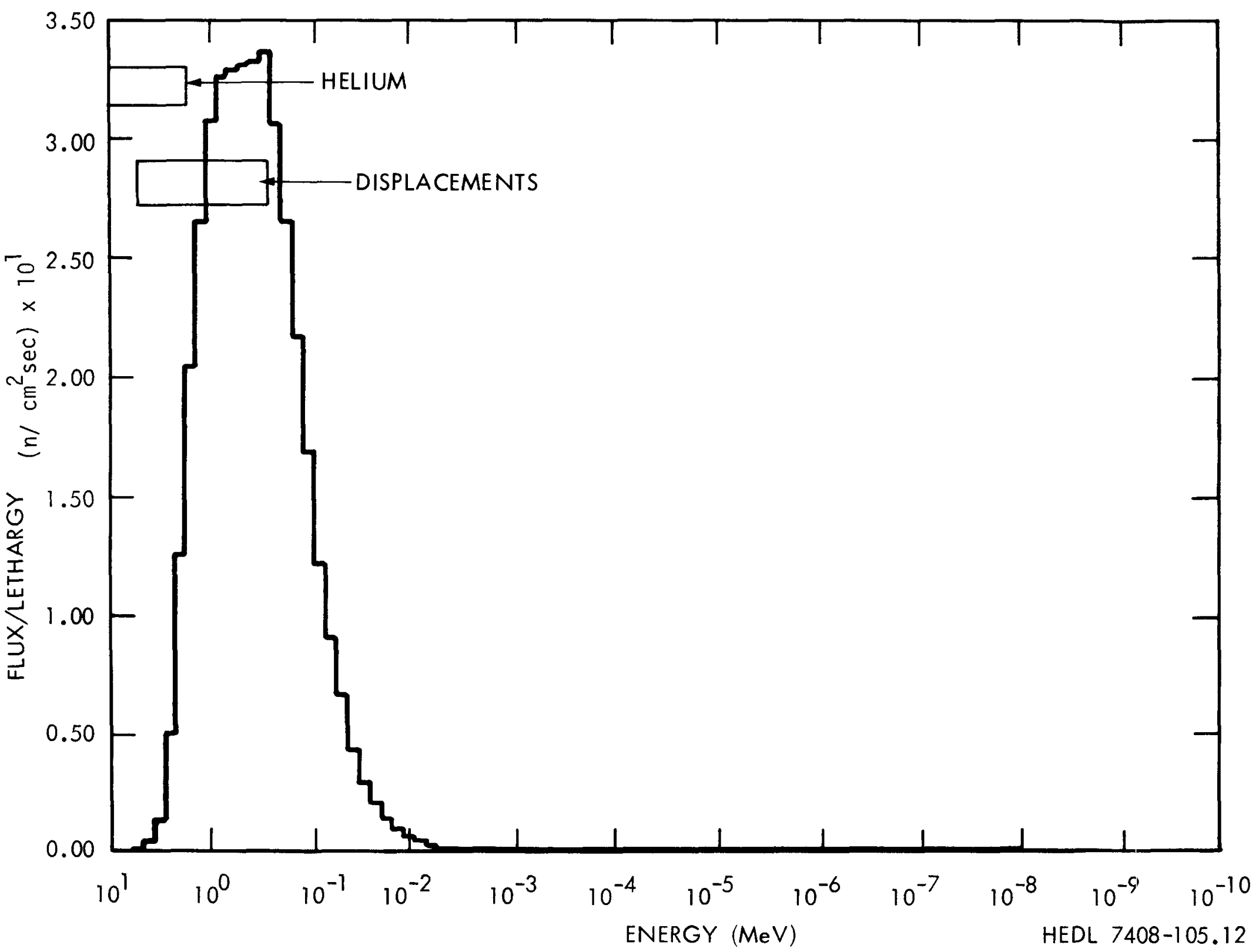

FIGURE C-3. APFACC--Normalized Flux Per Unit Lethargy Versus Energy. 


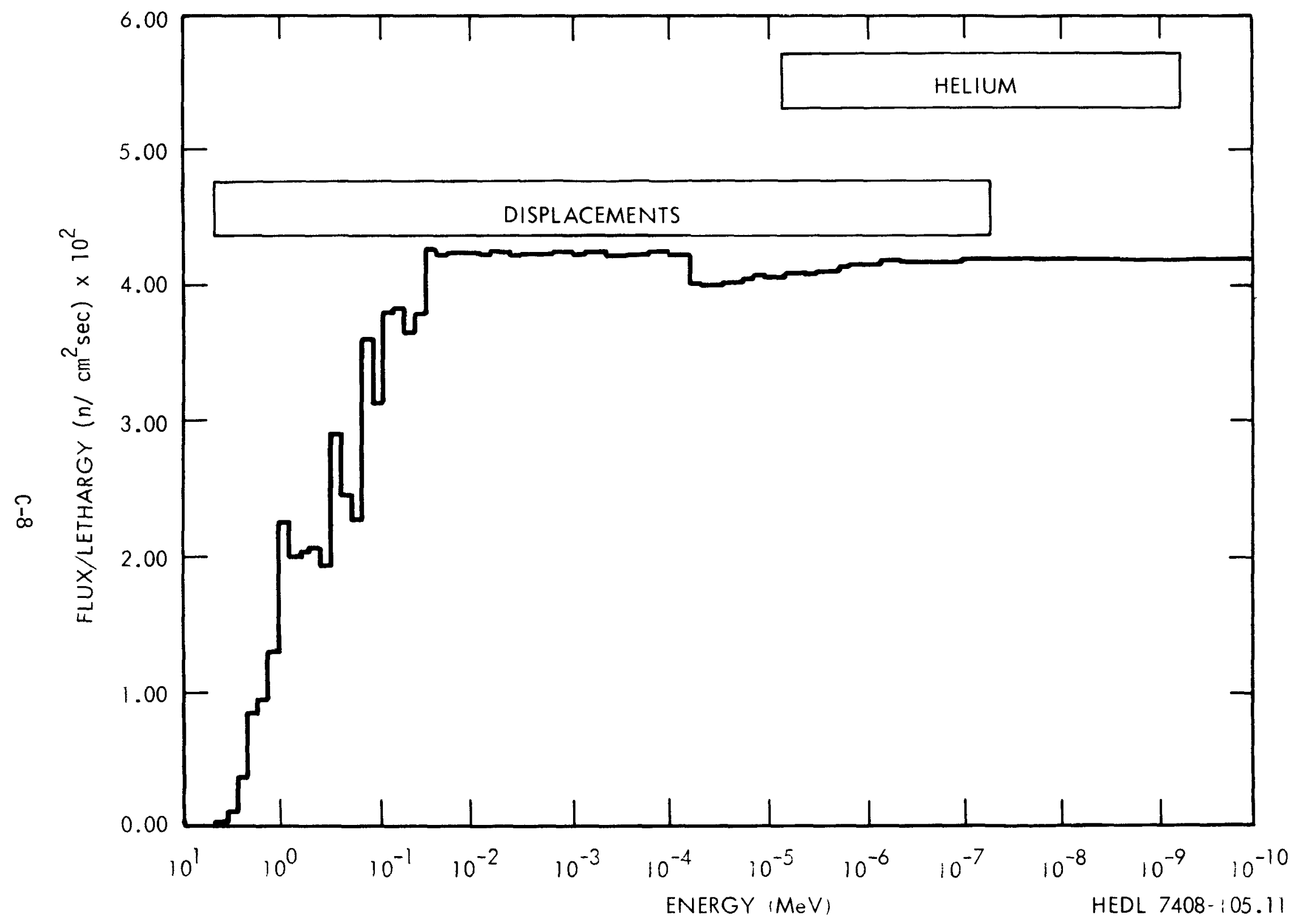

FIGURE C-4. EP5DUM--Normalized Flux Per Unit Lethargy Versus Energy. 


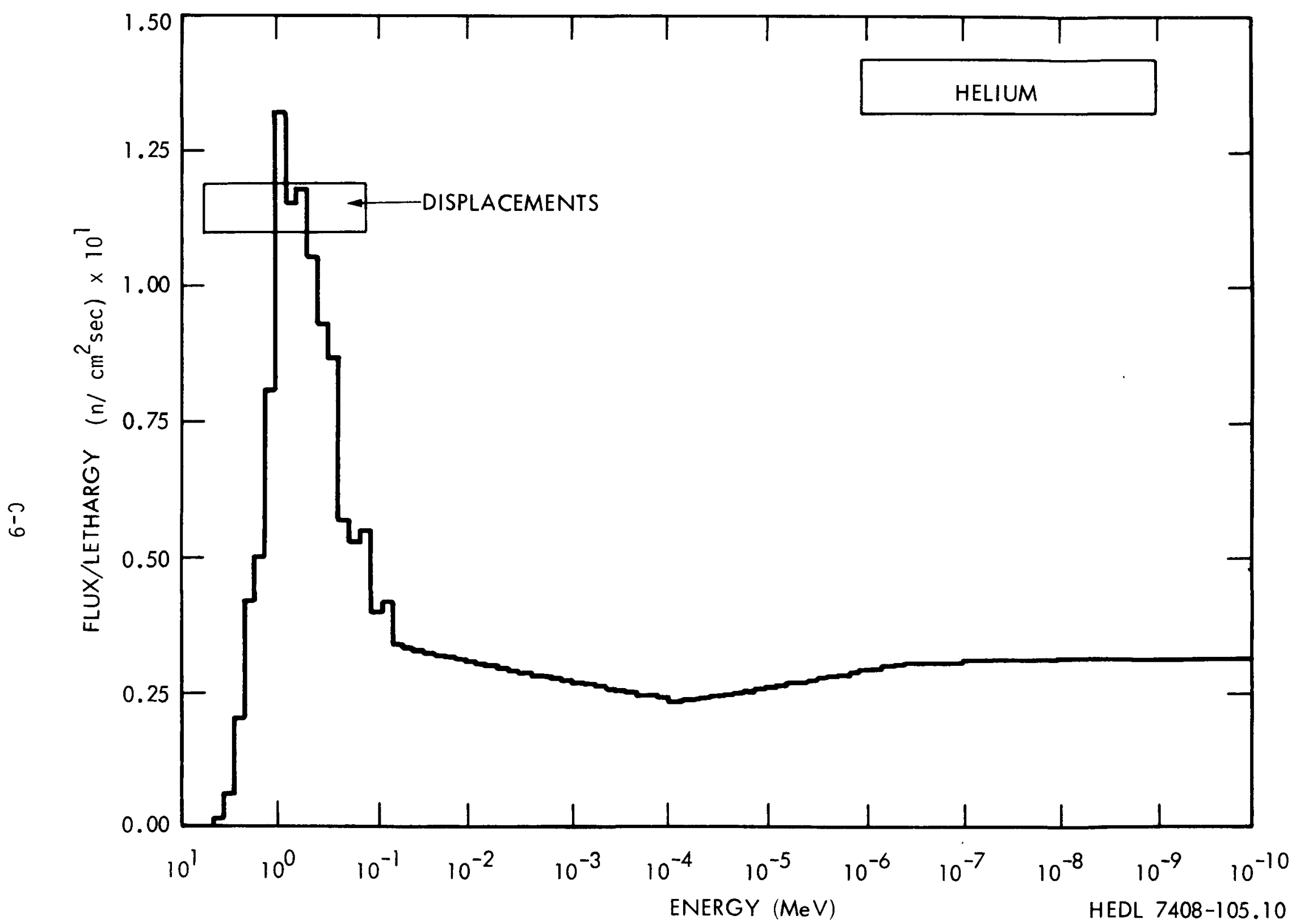

FIGURE C-5. ETR--Normalized Flux Per Unit Lethargy Versus Energy. 


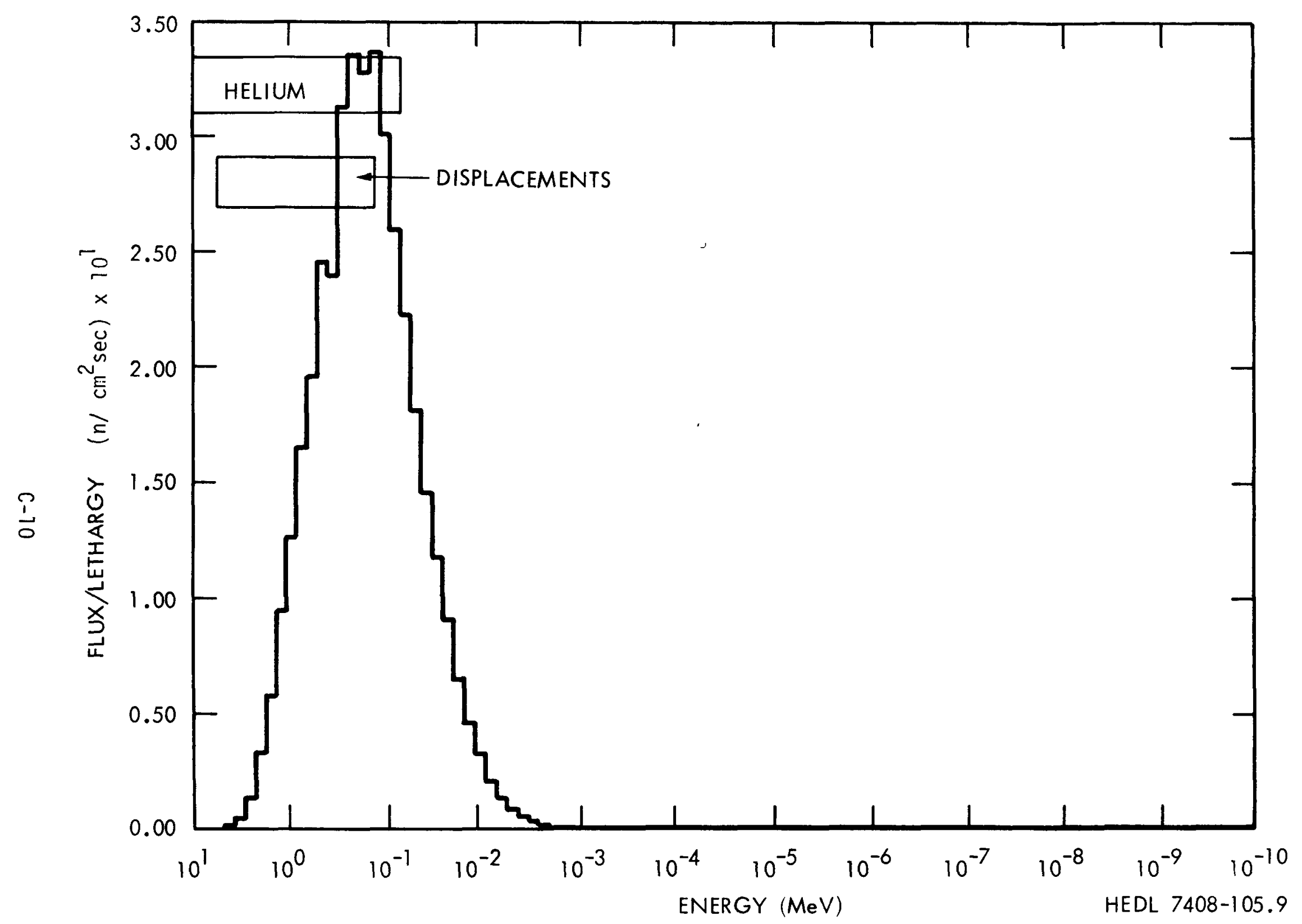

FIGURE C-6. EBR-A--Normalized Flux Per Unit Lethargy Versus Energy. 


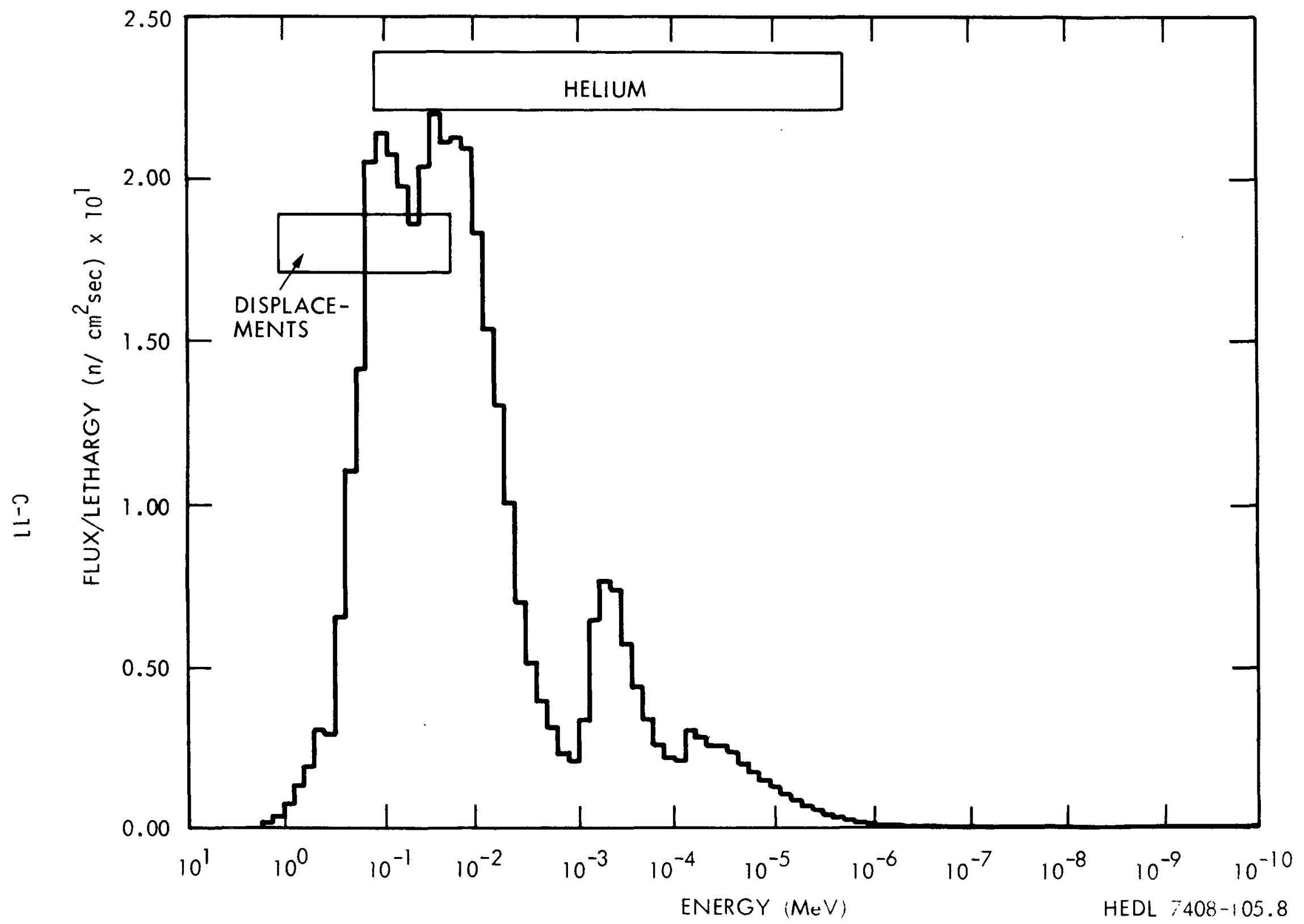

FIGURE C-7. EBR-D--Normalized Flux Per Unit Lethargy Versus Energy. 


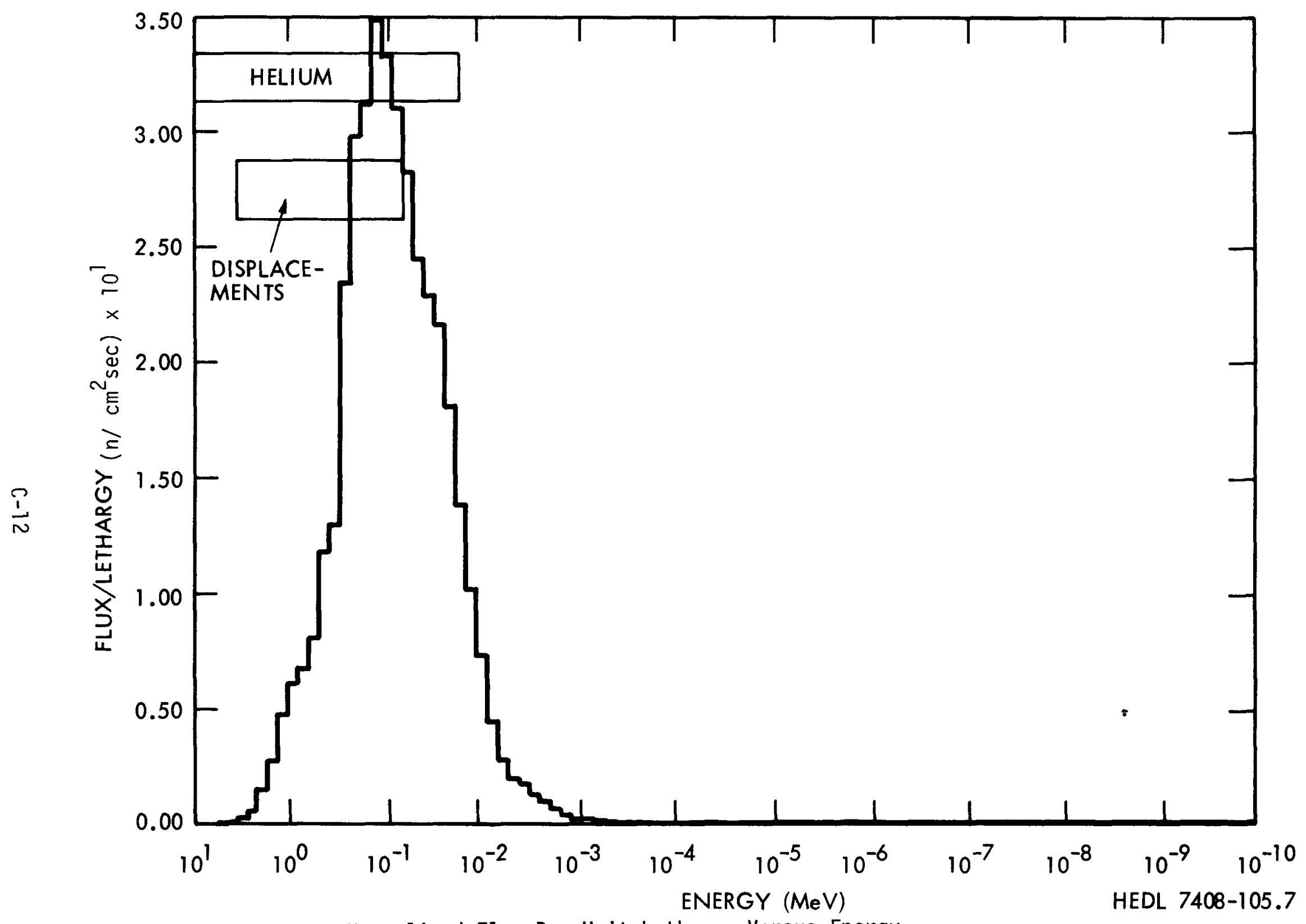

FIGURE C-8. EBR-B--Normalized Flux Per Unit Lethargy Versus Energy. 


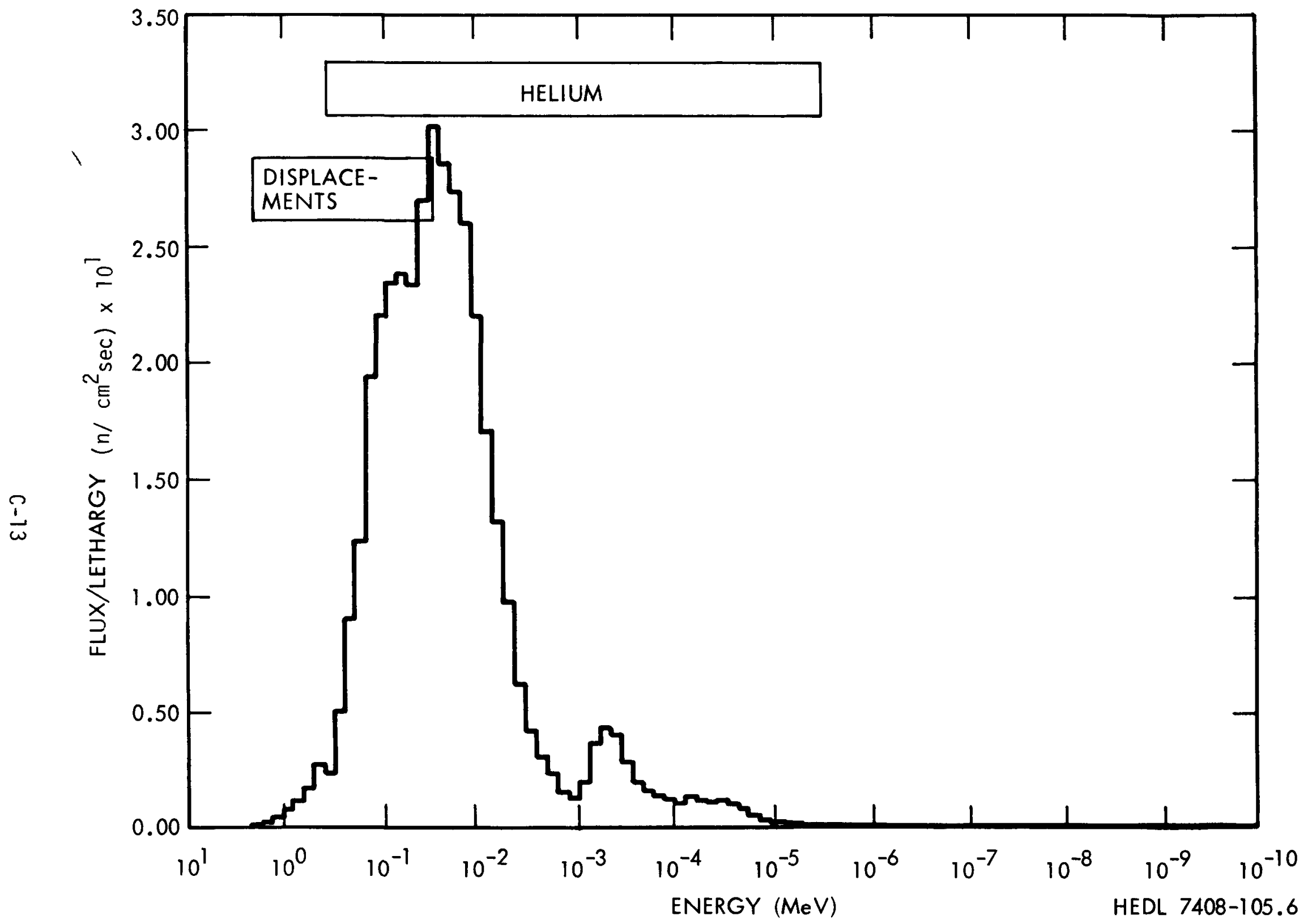

FIGURE C-9. EBR-C--Normalized Flux Per Unit Lethargy Versus Energy. 


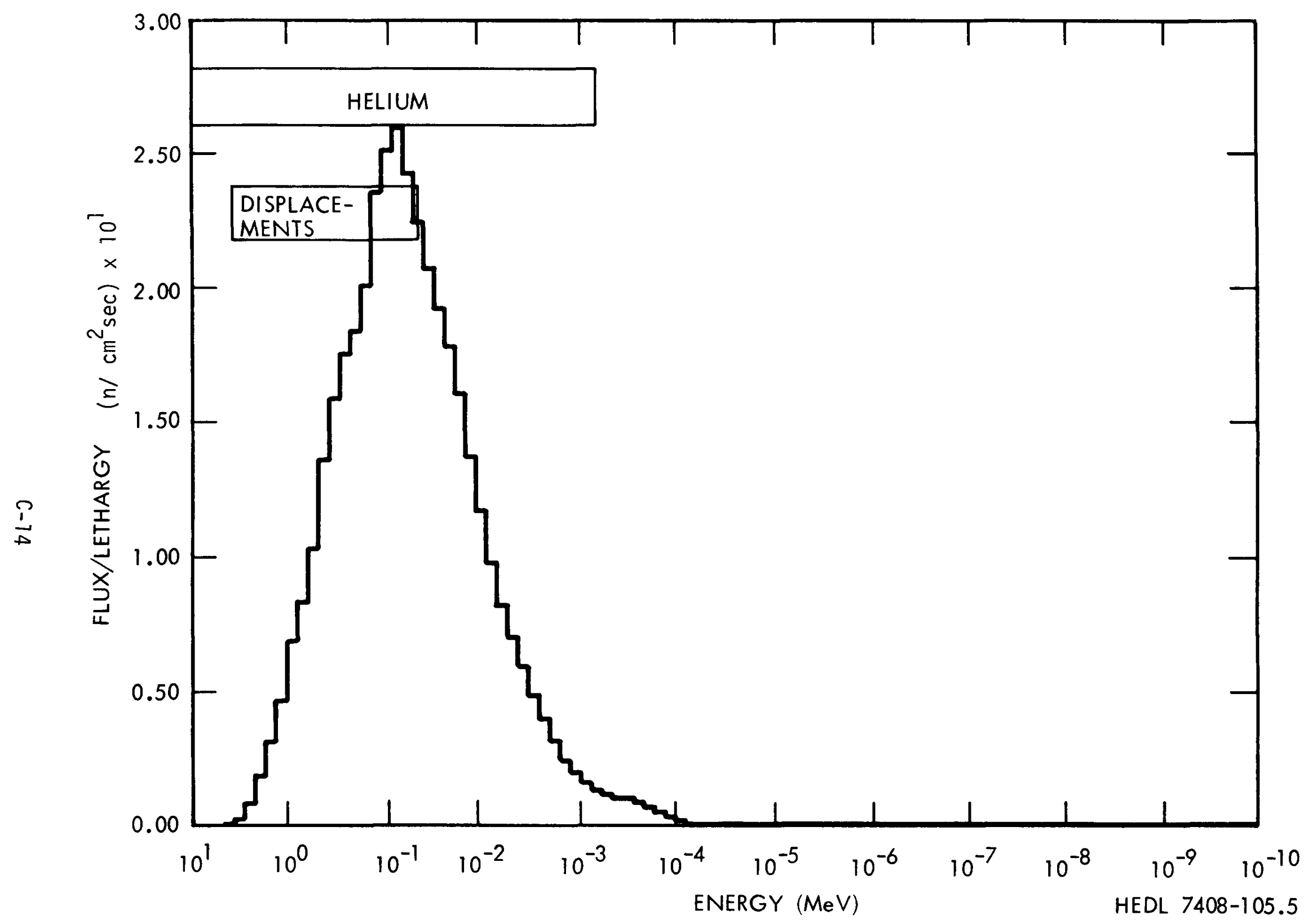

FIGURE C-10. FTRCORE--Normalized Flux Per Unit Lethargy Versus Energy. 


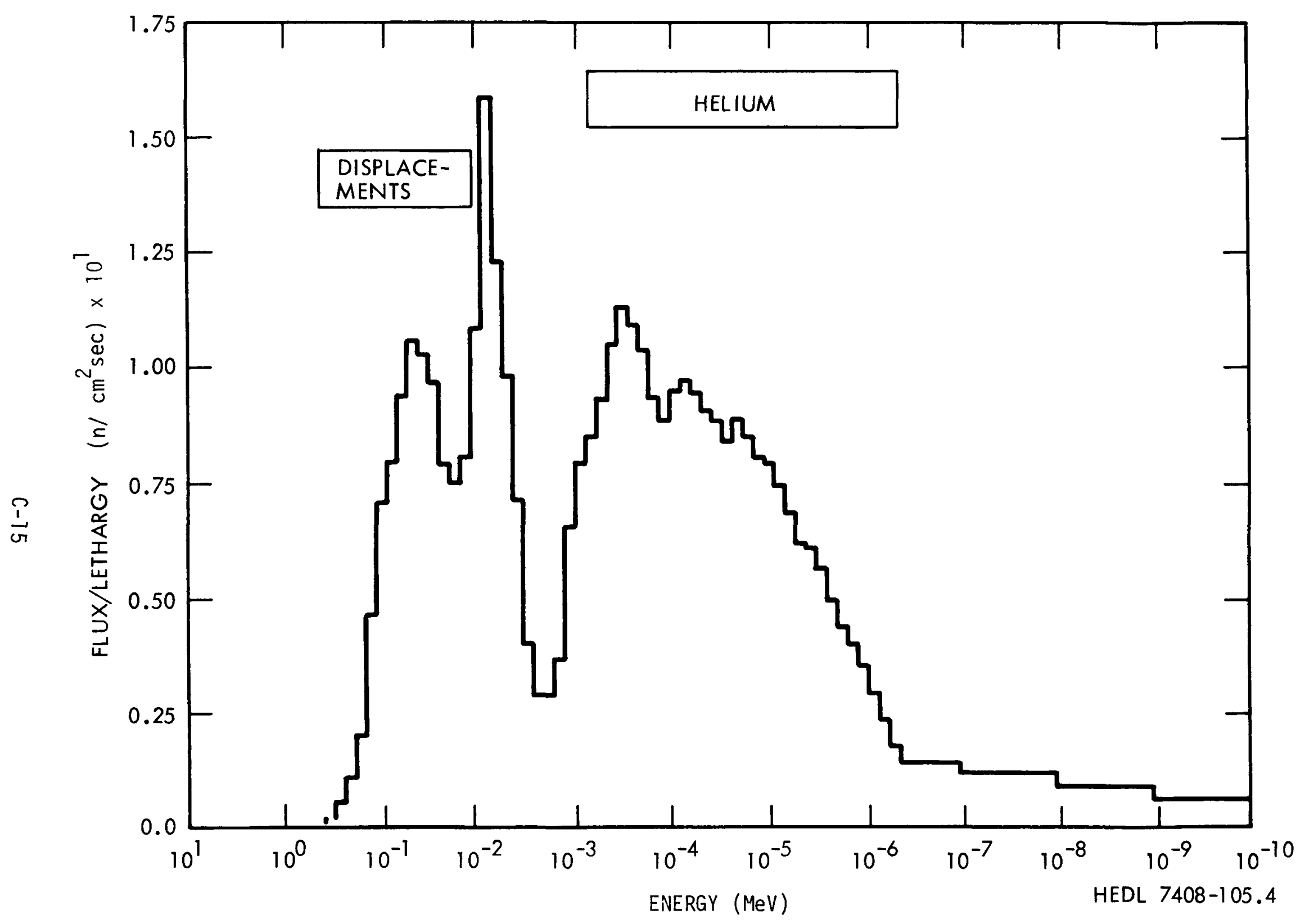

FIGURE C-11. FTRGDP--Normalized Flux Per Unit Lethargy Versus Energy. 


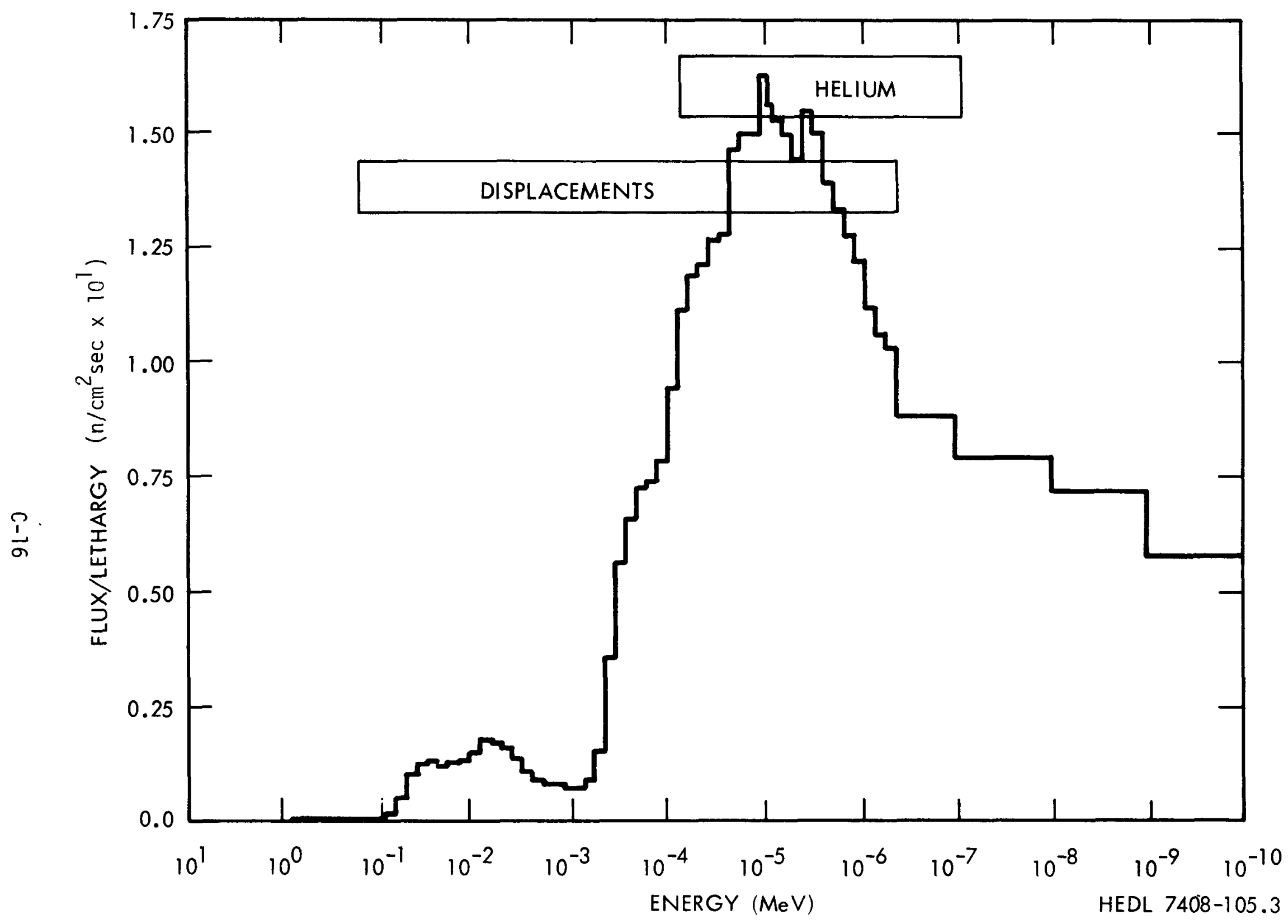

FIGURE C-12. FTRVESS--Normalized Flux Per Unit Lethargy Versus Energy. 


\section{APPENDIX D}

SOME TRIAL SOLUTIONS FOR

DISPLACEMENT AND HELIUM GENERATION PROCESSES 
In the text, it was pointed out that the solution damage function is dependent on the input approximation. There are a number of variables which can be considered when selecting the input approximation. Figure D-1 shows two displacement cross sections. (18) The difference between the two cross sections is the minimum displacement threshold. In one case, the value of $40 \mathrm{eV}$ was used, while in the second case $10 \mathrm{keV}$ was used. The latter could be associated with the formation of large clusters in a displacement cascade. This could, for example, be important in pinning glissile dislocations which would affect the yield strength.

Another energy dependent effect is obtained by computer simulation shown in Figure D-2. The displacement cascade produces a large number of displacements which undergo rapid annealing. The total number of residual defects given by a particular annealing model is described by the upper curve. Many of the vacancies produced in a high energy cascade are contained in immobile clusters, and hence the net number of mobile vacancies increases less rapidly with energy as shown in the lower curve. At sufficiently high temperatures, all vacancy clusters dissolve and only the total number of defect pairs will be important.

If both helium and displacements are important damage mechanisms, then a combination of the two defect sources could be linearly combined to produce a suitable input approximation. Figures $D-3$ and D-4 show $(n, \alpha)$ cross sections for Type $316 \mathrm{SS}$ with 1 ppma boron and the Fe- ${ }^{10} \mathrm{~B}$ cross section used in the interactive model described in the text.

Other energy dependent cross sections which should be considered for very soft spectra are the $(n, \gamma)$ recoil displacements and the two stage nickel reaction for production of helium; i.e., ${ }^{58} \mathrm{Ni}(\mathrm{n}, \gamma)^{59} \mathrm{Ni}(\mathrm{n}, \alpha){ }^{56} \mathrm{Fe}$.

The response function for the displacement and helium production cross section of SS are shown in Figures D-5 and D-6 for the three FTR spectra. It is evident that the bulk of the displacement damage is due to high energy neutrons, although there is a small contribution in the vessel spectrum due to low energy neutrons. The helium production is due primarily to low and intermediate energy neutrons in the grid plate and vessel spectrum. 
Figure D-7 shows the response of the displacement and helium cross sections for the ETR spectrum. Displacements are due to high energy neutrons while helium production is due to thermal neutrons. 


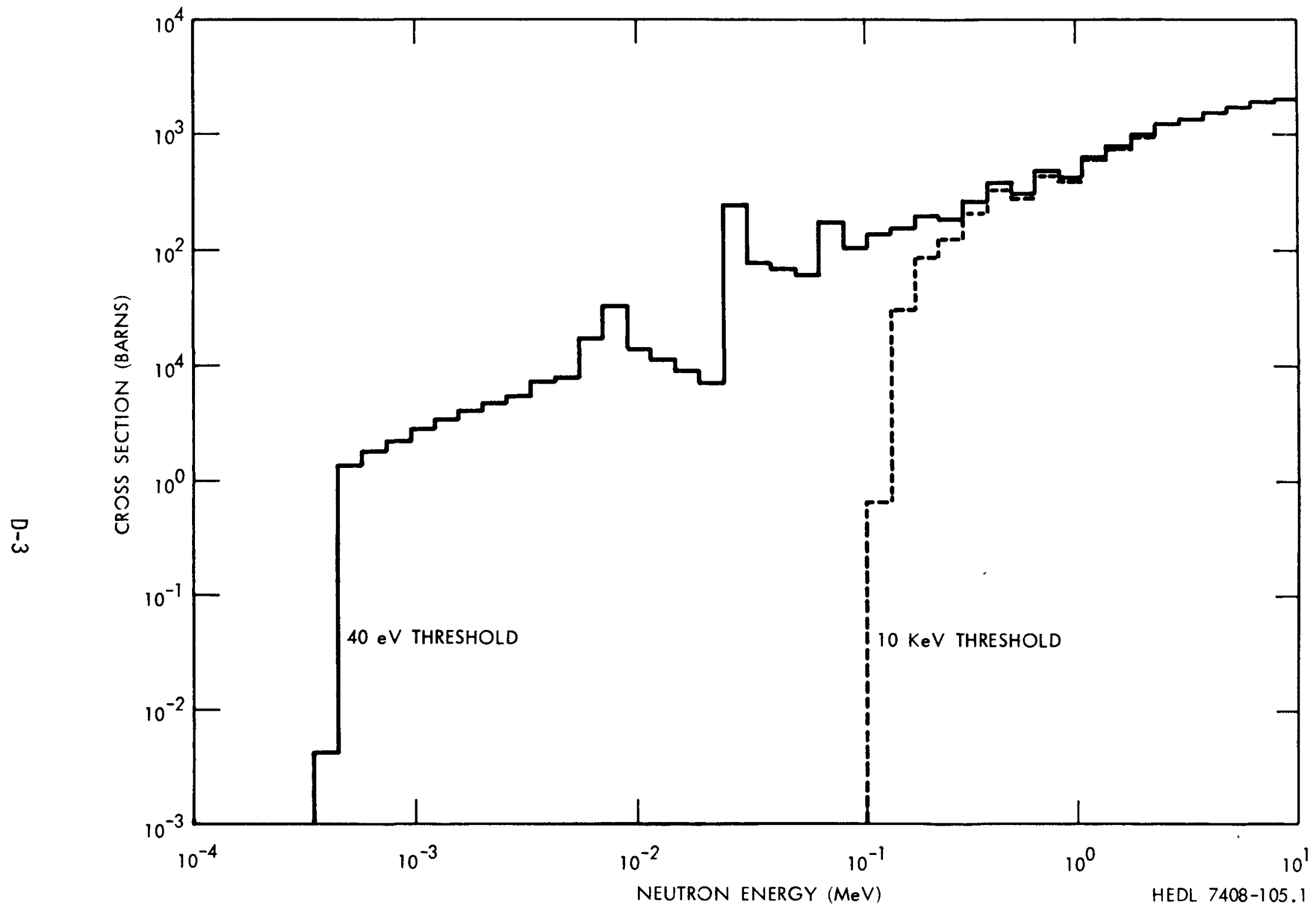

FIGURE D-1. Displacement Cross Section for $18 / 8(\mathrm{Cr} / \mathrm{Ni})$ Stainless Steel Using $40 \mathrm{eV}$ and $10 \mathrm{keV}$ Thresholds. 


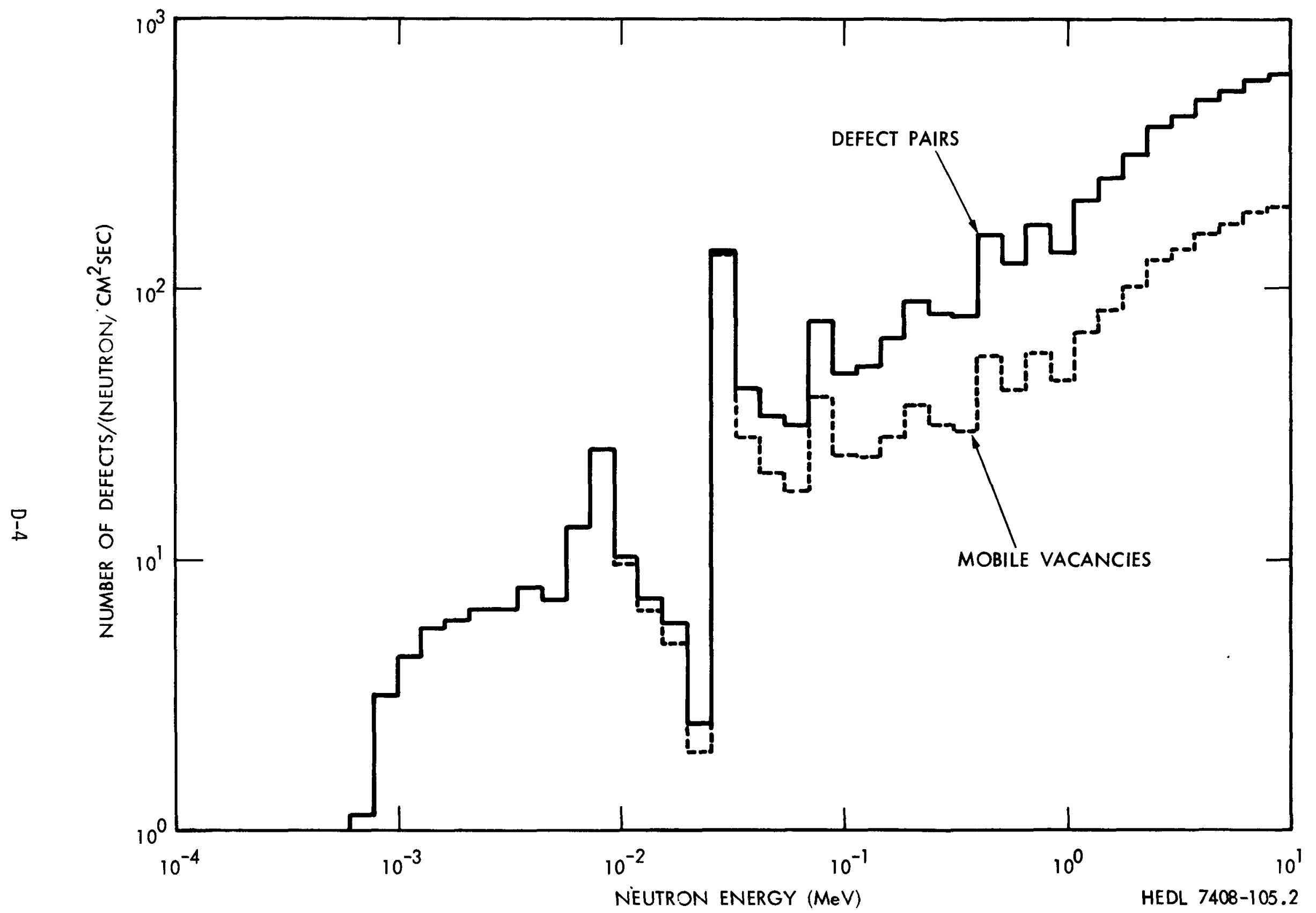

FIGURE D-2. Residual Defect Cross Sections for Stainless Steel. 


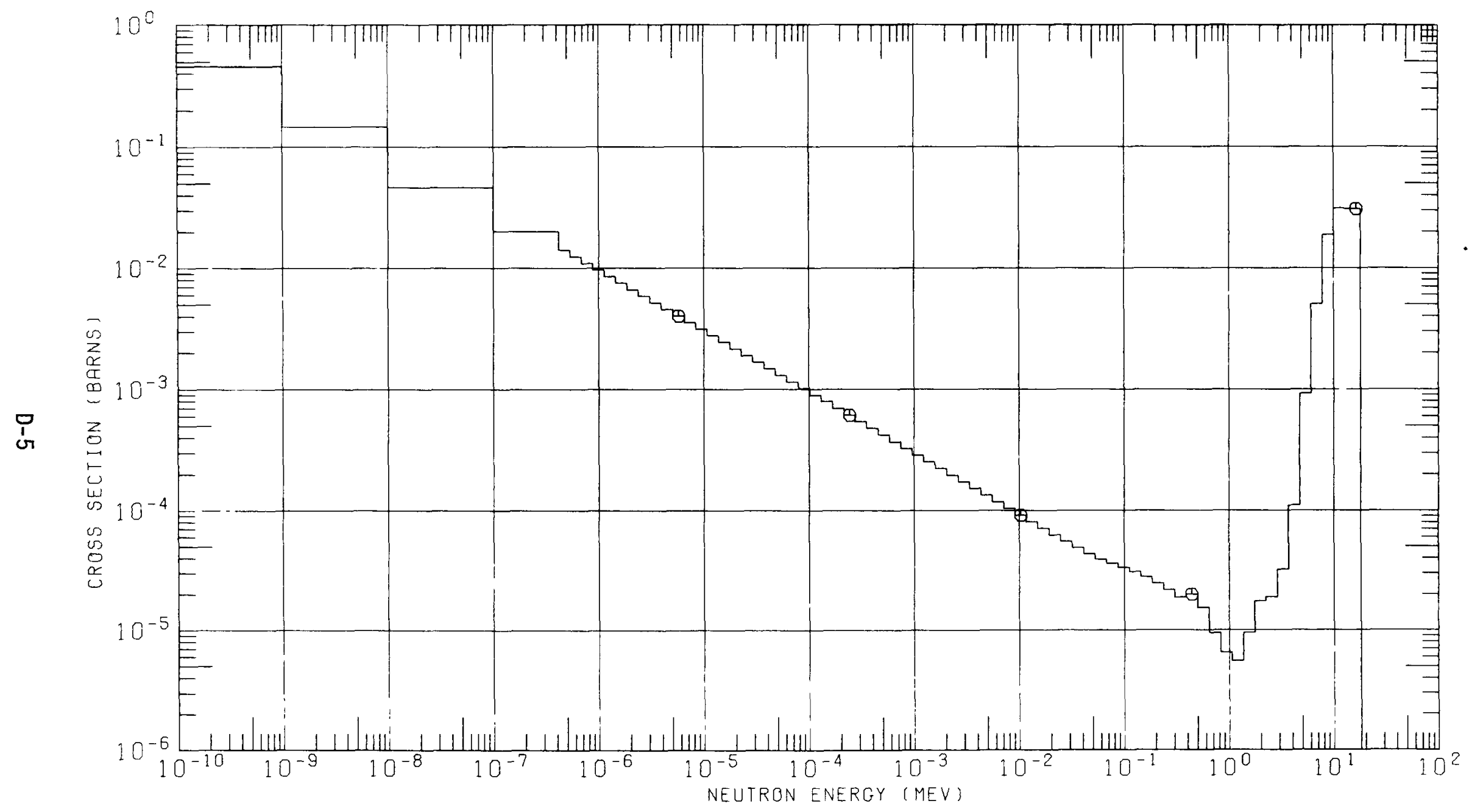

FIGURE D-3. Type 316 Stainless Steel $(n, \alpha)$ Cross Sections. 


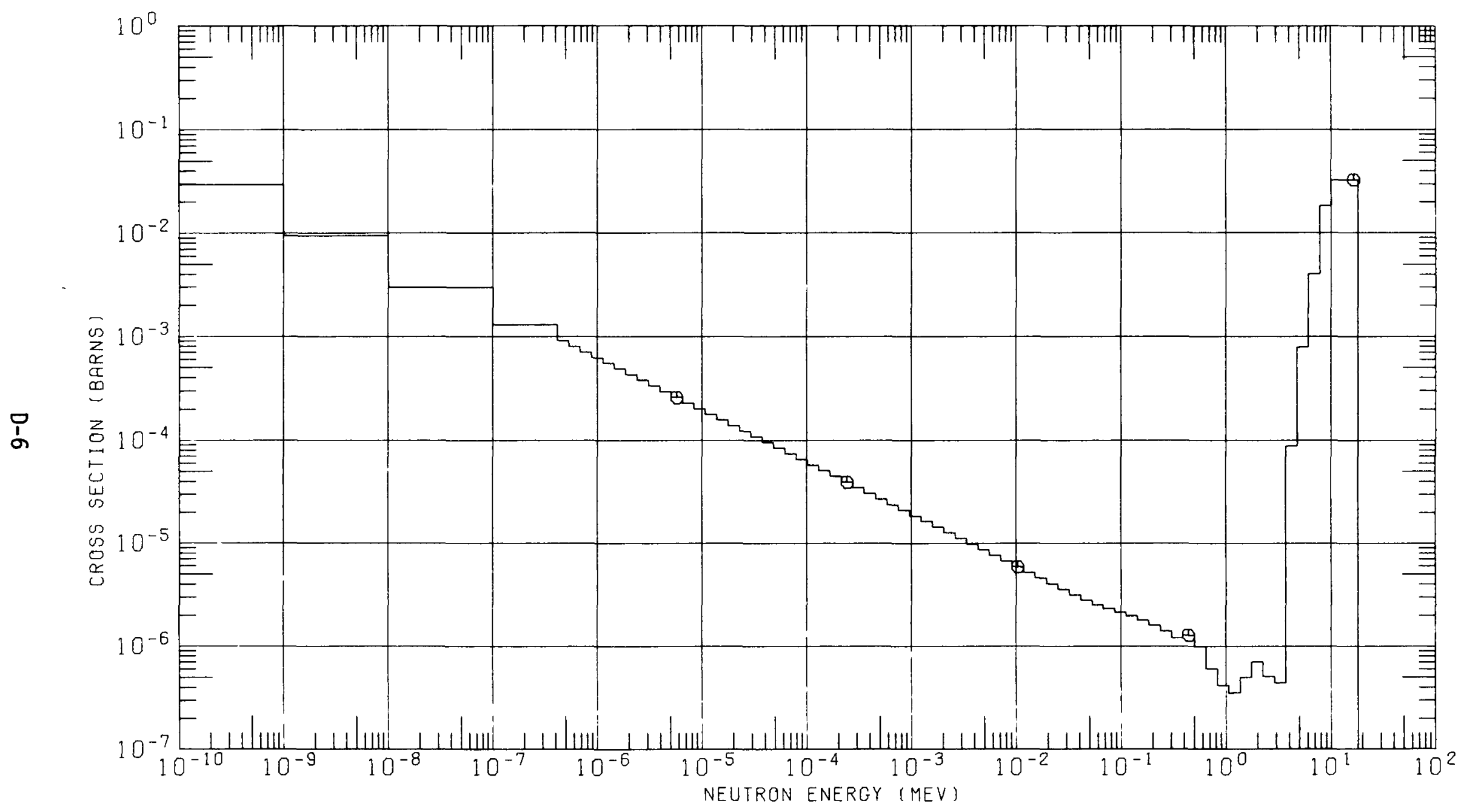

FIGURE D-4. $\mathrm{Fe}^{-10^{10}}(n, \alpha)$ Cross Section. 


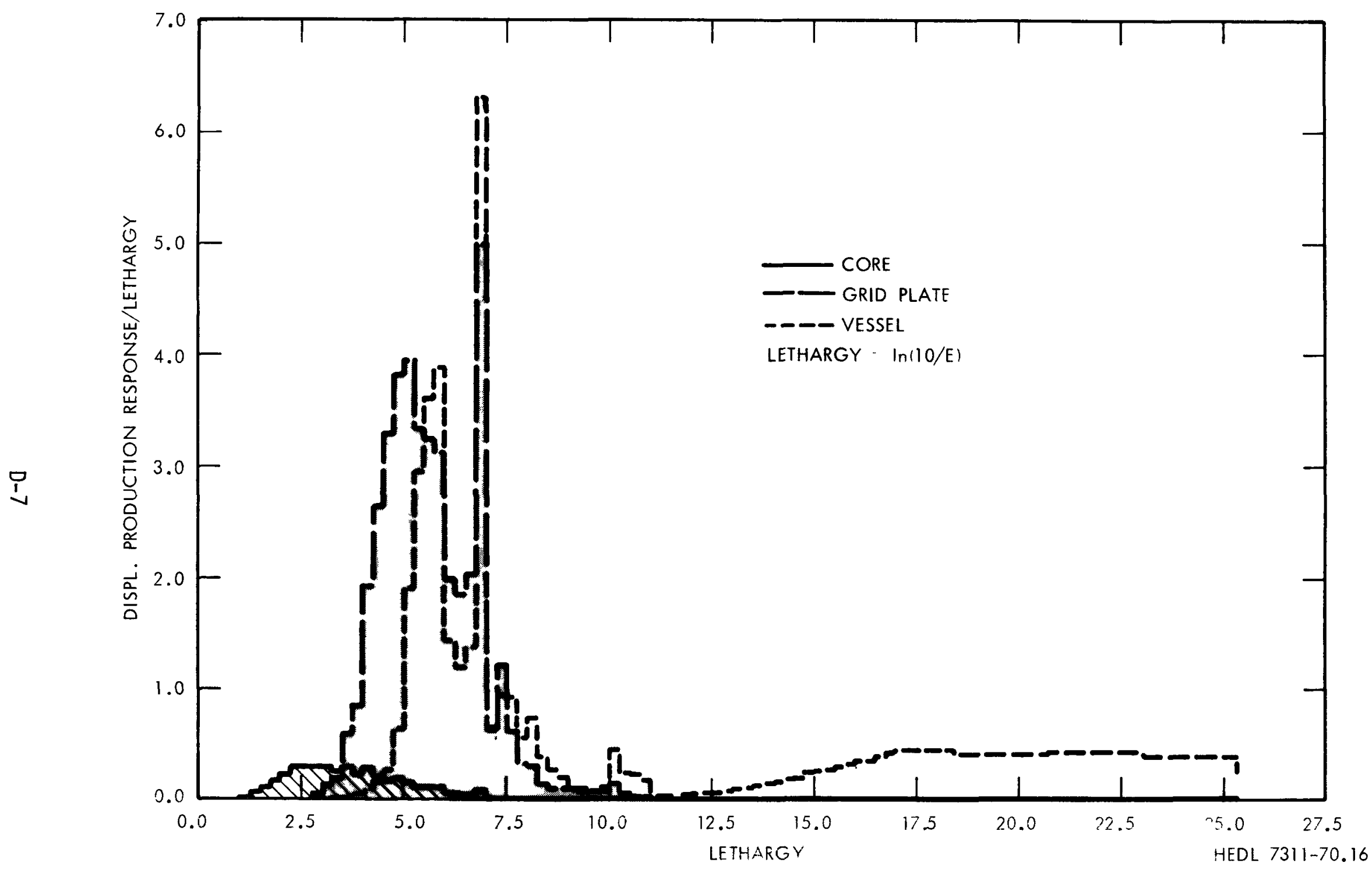

FIGURE D-5. Stainless Steel Displacement Production Response Functions Versus Lethargy for the Three FTR Design Spectra. 


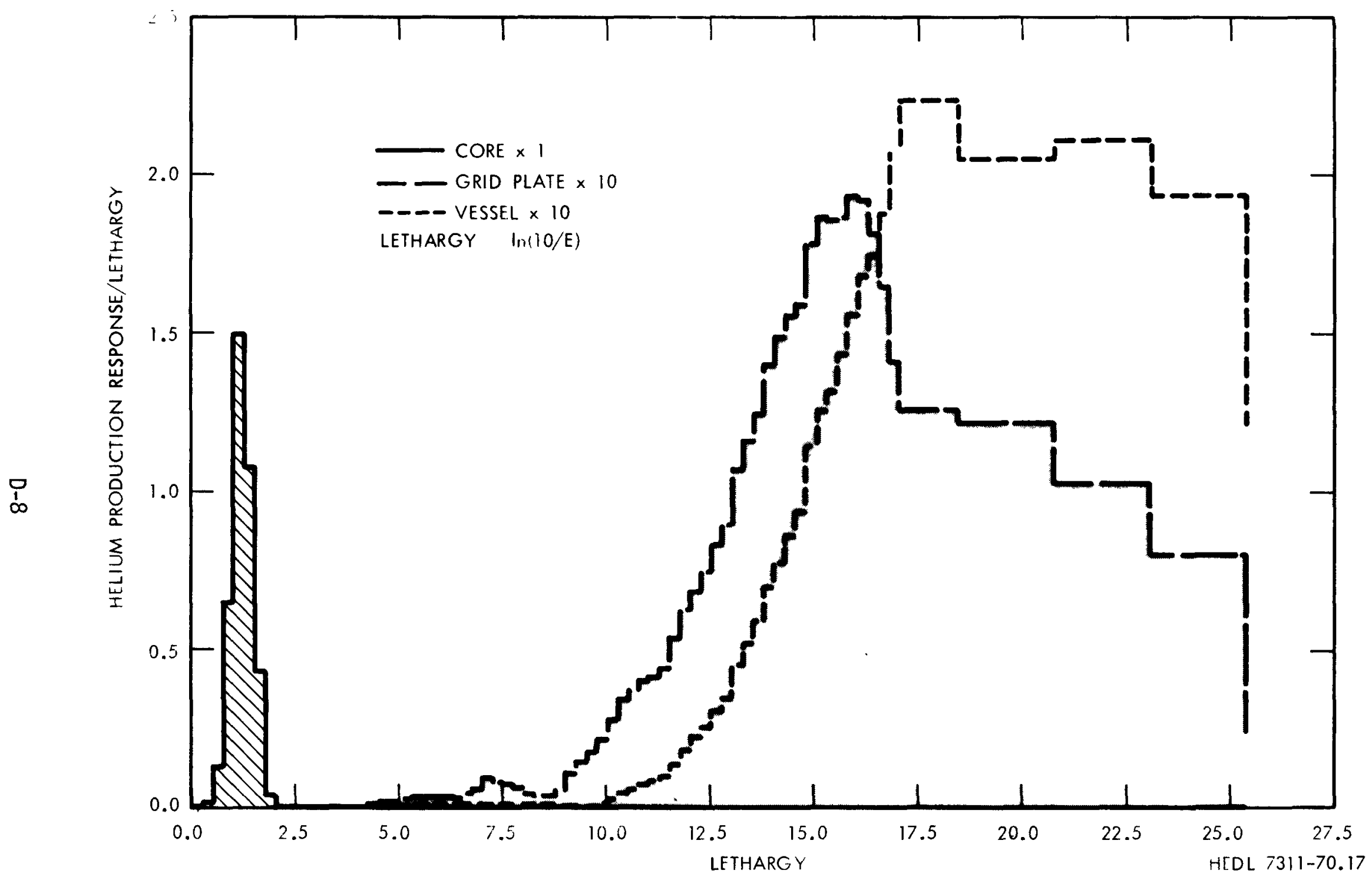

FIGURE D-6. Stainless Steel Helium Production Response Functions Versus Lethargy for the Three FTR Design Spectra. 


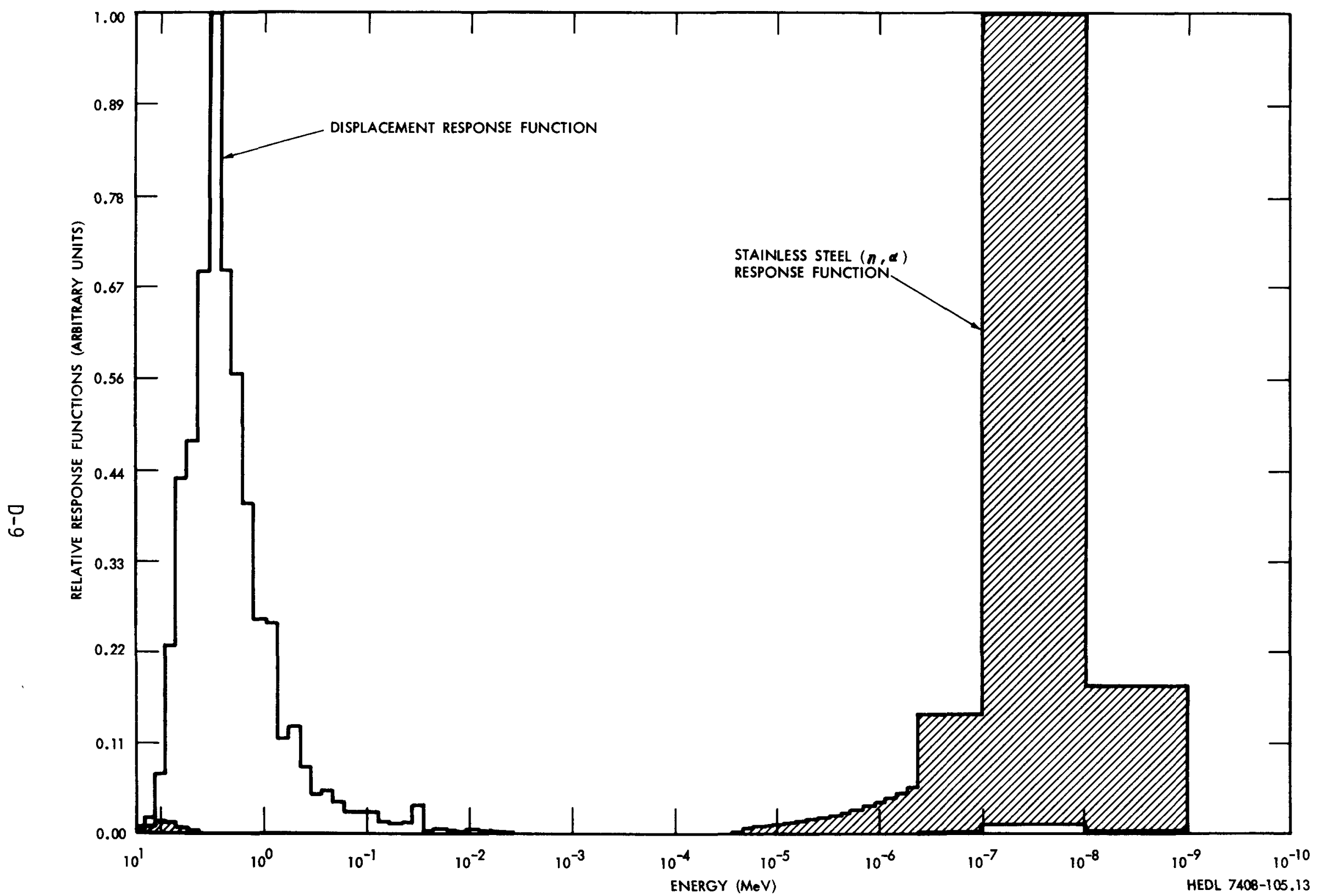

FIGURE D-7. Stainless Steel Helium and Displacement Response Functions Versus Energy for the ETR Design Spectrum. 
The parametric studies of the damage function error analysis in this study were performed to understand the error analysis per se. Consequently, the errors chosen were correct only in order of magnitude. EDF error analyses using actual data and appropriate errors have been carried out. One case involved yield strength changes in iron and another case involved yield strength changes in Type 304 stainless steel. The details are given in References 4 and 5, respectively.

The uniqueness uncertainty study on the 304 stainless steel EDF for yield strength involved starting the unfolding procedure from two extreme functions: (1) a constant for all energies and, (2) a form proportional to energy below $10 \mathrm{MeV}$ and a constant above $10 \mathrm{MeV}$. The results showed that the EDF was relatively insensitive to the starting function in the energy range $10^{-3}$ to $5 \mathrm{MeV}$.

The data errors were propagated by the Monte Carlo technique. The yield stress uncertainty was estimated at $\pm 6 \%(1 \sigma)$ and the fluence uncertainty $\pm 14 \%(1 \sigma)$ in EBR-II spectra and $\pm 19 \%(1 \sigma)$ in the thermal reactor spectrum. The combined errors (i.e., $\delta M_{j}$ ) were $16 \%$ and $20 \%$ for the fast and thermal spectra, respectively. The flux-spectra errors were distributed over four energy groups as shown in Table E-1. The one standard deviation total errors were calculated relative to the base solution.

Total solution errors were determined by summing the solution uniqueness error with the solution data error. ${ }^{(20)}$ The percent error in the damage function is shown in Table E-2 along with the damage function, group averaged using the ETR spectrum. The uncertainties in the fluence limit predictions for several spectra, as determined by the upper bound to the yield strength damage function, are shown in Table E-3. It is seen that the uniqueness uncertainty is small for these spectra. For softer spectra such as the FTR vessel wall, the uniqueness uncertainty is generally larger than the Monte Carlo error.

Details of an EDF error analysis for the low temperature, neutron induced yield stress changes in iron are given in Reference 4 . Uniqueness 
errors were determined from the envelope of solutions obtained from a set of input trial functions ranging from a constant to a form proportional to energy. In the energy region from $\sim 10^{-6}$ to $10^{-2} \mathrm{MeV}$, where there is little damage response, the slope of the total displacement function $\mathrm{G}_{\mathrm{D}}(\mathrm{E})$ was used to interpolate the upper bound of the EDF. The bounds of the resultant solution envelope were taken to be one standard deviation uniqueness errors.

Data errors were propagated using the Monte Carlo technique. Assuming a normal distribution, the $2 \sigma$ errors assigned were $\pm 6 \%$ to yield stress change; and $\pm 13 \%$ to total exposure. Spectral shape uncertainties were defined through total flux magnitudes in the several energy regions as follows: Energy range in MeV ( \pm error): $0-10^{-3}(30), 10^{-3}-10^{-2}(30)$, $10^{-2}-10^{-1}(30), 10^{-1}-0.6(40), 0.6-1.4(30), 1.4-2.2(25), 2.2-4(20), 4-6$ (20), 6-8 (20), 8-18(20).

The base solution was that which resulted from a trial form of a vacancy cluster formation cross section since this provided the best fit to the unperturbed data and converged to a solution most rapidly. Positive and negative errors were statistically calculated with reference to the base solution.

Total solution error was determined by summing uniqueness uncertainty and data error contributions. Table E-4 shows the damage function and \pm lo uncertainties in a 25 group structure based on $1 / E$ flux group averaging. Table E-5 shows the nominal and lower bound fluence 1 imits required to attain $15 \mathrm{ksi}$ yield stress change in various spectra of interest. 
TABLE E-1

PERCENT ERRORS ASSIGNED TO EBR-II AND ETR SPECTRA

$\%$ Uncertainty in

Group Integral Fluxes

Group Lower Bound

Energy (MeV)

$\underline{E B R-I I^{\mathrm{a}}} \quad \underline{E B R-I^{\mathrm{b}}} \quad \underline{E T R}$

$10^{-10}$

$1000^{c}$

$300^{\mathrm{C}}$

10

$10^{-6}$

33

11

25

$10^{-1}$

11

19

40

1.0

8

16

15

Total Fluence Error

14

14

19

$a_{\text {Hard spectra in EBR-II. }}$

${ }^{b}$ Intermediate and soft spectra in EBR-II.

${ }^{\mathrm{C}}$ There is a relatively little flux in these groups and, consequently, they are subject to large uncertainty. 
TABLE E-2

GROUP AVERAGED (ETR SPECTRUM) DAMAGE FUNCTION FOR 60 KSI YIELD STRENGTH TYPE 304 SS AT $480^{\circ} \mathrm{C}$

\begin{tabular}{|c|c|c|c|}
\hline Group & $\begin{array}{c}\text { Lower } \\
\text { Energy } \\
(\mathrm{MeV})\end{array}$ & $\begin{array}{l}\text { 60-ksi Yield } \\
\text { Strength, } \bar{G}_{j} \\
{\left[k s i /\left(\mathrm{n} / \mathrm{cm}^{2}\right)\right]}\end{array}$ & $\begin{array}{l}+]_{\text {is }} \text { Percent } \\
\text { Errors } \\
\text { in } G(E) \\
\end{array}$ \\
\hline 1 & $7.79+00$ & $1.94-20$ & 220 \\
\hline 2 & $6.07+00$ & $2.16-20$ & 63 \\
\hline 3 & $4.72+00$ & $2.16-20$ & 34 \\
\hline 4 & $3.68+00$ & $2.13-20$ & 18 \\
\hline 5 & $2.87+00$ & $2.18-20$ & 7 \\
\hline 6 & $2.23+00$ & $2.02-20$ & 11 \\
\hline 7 & $1.74+00$ & $1.77-20$ & 7 \\
\hline 8 & $1.35+00$ & $1.52-20$ & 2 \\
\hline 9 & $1.05+00$ & $1.27-20$ & 9 \\
\hline 10 & $8.21-01$ & $1.07-20$ & 14 \\
\hline 11 & $6.39-01$ & $9.37-21$ & 7 \\
\hline 12 & 4.98-01 & $8.16-21$ & 1 \\
\hline 13 & 3.88-01 & $6.55-21$ & 1 \\
\hline 14 & $3.02-01$ & $5.29-21$ & 7 \\
\hline 15 & $2.35-01$ & $4.20-21$ & 16 \\
\hline 16 & $1.83-01$ & $3.67-21$ & 16 \\
\hline 17 & $1.43-01$ & $3.27-21$ & 13 \\
\hline 18 & $1.11-01$ & $2.83-21$ & 8 \\
\hline 19 & $6.74-02$ & $2.27-21$ & 1 \\
\hline 20 & $2.48-02$ & $1.68-21$ & 18 \\
\hline 21 & $9.12-03$ & $6.78-22$ & 11 \\
\hline 22 & $3.36-03$ & $2.50-22$ & 137 \\
\hline 23 & $1.23-03$ & $1.73-22$ & 200 \\
\hline 24 & $4.54-04$ & $4.79-23$ & 720 \\
\hline 25 & $1.67-04$ & $2.10-24$ & 17000 \\
\hline 26 & $6.14-05$ & $2.41-24$ & 13000 \\
\hline 27 & $2.26-05$ & $2.77-24$ & 12000 \\
\hline 28 & $8.32-06$ & $4.99-24$ & 7200 \\
\hline 29 & $3.06-06$ & $9.86-24$ & 3800 \\
\hline 30 & $1.12-06$ & $1.74-23$ & 2400 \\
\hline 31 & $4.14-07$ & $3.00-23$ & 1500 \\
\hline 32 & $1.00-10$ & 1. $22-22$ & 590 \\
\hline
\end{tabular}


TABLE E-3

ESTIMATED $\pm 2 \sigma$ UNCERTAINTIES IN PREDICTED FLUENCE REQUIRED

TO ATTAIN 60-KSI YIELD STRENGTH IN TYPE 304 SS AT $480^{\circ} \mathrm{C}$

$\%$ Fluence Uncertainty for 60-ksi Yield Strength

\section{Spectrum}

EBR-II core center

EBR-II blanket

ETR core

FTR core center

FTR grid plate
Mean Neutron

Energy

0.82

0.29

0.51

0.48

0.036

24

12

10

35

12
Monte

Carlo

10

20

2

37

2

14

E-5 
TABLE E-4

EMPIRICAL DAMAGE FUNCTION FOR IRON

Lower Group

Energy (MeV)

7.79

6.07

4.72

3.68

2.87

2.23

1.74

1.35

1.05

$8.21 \times 10^{-}$

$6.39 \times 10^{-1}$

$4.98 \times 10^{-1}$

$3.88 \times 10^{-1}$

$3.02 \times 10^{-1}$

$2.35 \times 10^{-1}$

$1.83 \times 10^{-1}$

$6.74 \times 10^{-2}$

$1.17 \times 10^{-2}$

$3.36 \times 10^{-3}$

$1.23 \times 10^{-3}$

$5.83 \times 10^{-4}$

$1.01 \times 10^{-4}$

$8.32 \times 10^{-6} 7$

$6.83 \times 10^{-7}$

0
Damage Function $\times 10^{16}$

$\left(\mathrm{ksi}^{2} / \mathrm{n} / \mathrm{cm}^{2}\right)$

73

78

78

75

66

57

49

42

32

23

20

18

17

13

11

10

6

0.26

0

0

0

0

0

0

0
Damage Function ${ }^{a}$ Uncertainty $\times 10^{16}$ $\left(\mathrm{ksi}^{2} / \mathrm{n} / \mathrm{cm}^{2}\right)$

$\begin{array}{rr}+150 & -40 \\ +70 & -25 \\ +48 & -18 \\ +18 & -11 \\ +10 & -8\end{array}$

$+4 \quad-6$

$+2-20$

$+4 \quad-12$

$+2-8$

$+8-5$

$+6 \quad-4$

$+5-7$

$+6 \quad-8$

$+6-8$

$+3 \quad-9$

$+5 \quad-8$

$+4 \quad-4$

$+1.7-0.2$

$+0.4-0$

$+0.06-0$

$+0.02-0$

$+0.002-0$

$+0.004-0$

$+0.010-0$

$+0.075-0$

a. One standard deviation estimates. 
TABLE E-5

EXPOSURES NEEDED TO PRODUCE 15 KSI YIELD STRENGTH CHANGE IN IRON AT ROOM TEMPERATURES $\times 10^{-19} \cdot\left(\mathrm{n} / \mathrm{cm}^{2}\right)$

Reactor Spectrum

Correlation

Technique

FTR Grid Plate FTR Vessel FTR Core ETR Core

Damage Function

9.0

160

.94

1.1

Damage Function

+ To Error

5.2

68

.73

0.90 